\title{
- Coverage Probability and Achievable Rate Analysis of FFR-Aided Multi-User OFDM-Based MIMO and SIMO Systems
}

5 Abstract-Expressions are derived for the coverage probability 6 and average rate of both multi-user multiple input multiple output 7 (MU-MIMO) and single input multiple output (SIMO) systems 8 in the context of a fractional frequency reuse (FFR) scheme. In 9 particular, given a reuse region of $\frac{1}{3}(\mathrm{FR3})$ and a reuse region of 101 (FR1) as well as a signal-to-interference-plus-noise-ratio (SINR) 11 threshold $S_{t h}$, which decides the user assignment to either the FR1 12 or FR3 regions, we theoretically show that: 1) the optimal choice 13 of $S_{t h}$ which maximizes the coverage probability is $S_{t h}=T$, where $14 \boldsymbol{T}$ is the target SINR required for ensuring adequate coverage, and 152 ) the optimal choice of $S_{t h}$ which maximizes the average rate is 16 given by $S_{t h}=T^{\prime}$, where $T^{\prime}$ is a function of the path loss exponent, 17 the number of antennas and of the fading parameters. The impact 18 of frequency domain correlation amongst the OFDM sub-bands 19 allocated to the FR1 and FR3 cell-regions is analysed and it is 20 shown that the presence of correlation reduces both the coverage 21 probability and the average throughput of the FFR network. 22 Furthermore, the performance of our FFR-aided MU-MIMO and 23 SIMO systems is compared. Our analysis shows that the $(2 \times 2)$ 24 MU-MIMO system achieves $22.5 \%$ higher rate than the $(1 \times 3)$ 25 SIMO system and for lower target SINRs, the coverage probability 26 of a $(2 \times 2)$ MU-MIMO system is comparable to a $(1 \times 3)$ SIMO 27 system. Hence the former one may be preferred over the latter. 28 Our simulation results closely match the analytical results.

29 Index Terms-Author, please supply index terms/keywords for 30 your paper. To download the IEEE Taxonomy go to http://www. 31 ieee.org/documents/taxonomy_v101.pdf.

\section{INTRODUCTION}

33

34 RTHOGONAL frequency division multiple access (OFDMA) based systems maintain orthogonality among 35 the intra-cell users, but the radical OFDMA system deploy36 ments relying on a frequency reuse factor of unity suffer from 37 inter-cell interference. As a remedy, inter-cell interference coor38 dination (ICIC) schemes have been designed for minimizing the 39 co-channel interference [1]. Fractional frequency reuse (FFR) 40 [2] constitutes a low complexity ICIC scheme, which has been 41 proposed for OFDMA based wireless networks such as IEEE 42 WiMAX [3] and 3GPP LTE [4].

Manuscript received January 18, 2015; revised June 5, 2015; accepted August 1,2015. The associate editor coordinating the review of this paper and approving it for publication was O. Oyman.

S. Kumar, S. Kalyani, and K. Giridhar are with the Indian Institute of Technology Madras, Chennai 600 036, India (e-mail: ee10d040@ee.iitm.ac.in; skalyani@ee.iitm.ac.in; giri@ee.iitm.ac.in).

L. Hanzo is with the School of Electrical and Computer Science, University of Southampton, Southampton SO17 1BJ, U.K. (e-mail: lh@ecs.soton.ac.uk).

Color versions of one or more of the figures in this paper are available online at http://ieeexplore.ieee.org.

Digital Object Identifier 10.1109/TCOMM.2015.2465907

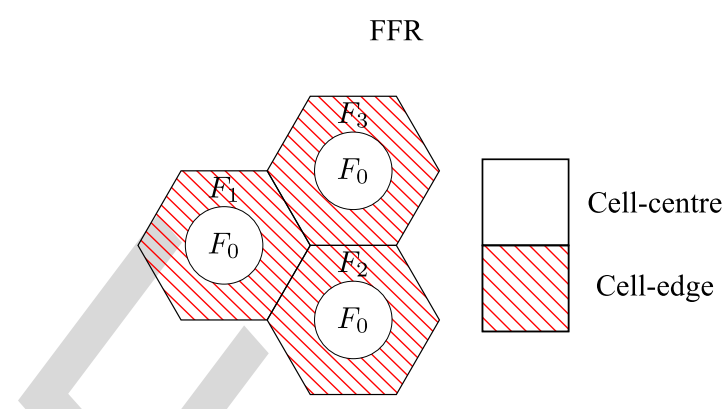

Fig. 1. Frequency allocation in FFR for three neighbouring cells with $\delta=3$. The cell-centre users of all the cells rely on a common frequency band $F_{0}$, while the cell-edge users of the three cells occupy different frequency bands, namely $F_{1}, F_{2}$ and $F_{3}$.

Explicitly, FFR is a combination of frequency reuse 1 (FR1) 43 and frequency reuse $\frac{1}{\delta}(\mathrm{FR} \delta)$. FR1 allocates all the frequencies 44 to each cell, leading to a unity spatial reuse, hence results in 45 a low-quality coverage due to the excessive inter-cell interfer- 46 ence. On the other hand, FR $\delta$ allocates a fraction of $\frac{1}{\delta}$ of the 47 frequencies to each cell and therefore reduces the area-spectral- 48 efficiency, but improves the SINR. FFR strikes an attractive 49 trade-off by exploiting the advantages of both FR 1 and FR $\delta$ by 50 relying on FR1 for the cell-centre users i.e. for those users who 51 would experience less interference from the other cells, because 52 they are close to their serving base station (BS). By contrast, 53 $\mathrm{FR} \delta$ is invoked for the cell-edge users i.e. for those users who 54 would experience high interference afflicted by the co-channel 55 signals emanating from the neighbouring cells in case of FR1, 56 because they are far from their serving BS. Typically, there 57 are two basic modes of FFR deployment: static and dynamic 58 FFR [1]. In this paper, we consider the more practical static 59 FFR scheme, where all the parameters are configured and kept 60 fixed over a certain period of time [5]. Fig. 1 depicts a typical 61 frequency allocation in the context of the FFR scheme for three 62 adjacent cells, where $F_{1}, F_{2}$ and $F_{3}$ each use $x \%$ of the total 63 spectrum, hence $F_{0}$ uses $(100-3 x) \%$ of the spectrum.

FFR schemes have been lavishly studied using both system 65 level simulations and theoretical analysis [6]-[11]. The optimiz- 66 ation of FFR relying on a distance threshold ${ }^{1}$ or SINR threshold ${ }^{2} 67$

\footnotetext{
${ }^{1}$ Based on a pre-determined distance from the BS, the subscribers are divided into cell-centre as well as cell-edge users and hence here the design parameter is a distance threshold $\left(R_{t h}\right)$.

${ }^{2}$ Based on a pre-determined SINR, the subscribers are divided into cellcentre as well as cell-edge users and here the design parameter is the SINR threshold $\left(S_{t h}\right)$.
} 
68 has been studied using graph theory in [6] and convex optimiza69 tion in [7]. Specifically, it has been shown in [7] that the optimal 70 frequency reuse factor is FR3 for the cell-edge users. The av71 erage cell throughput of an FFR system was derived in [8] as a 72 function of the distance threshold. It was shown in [9] that there 73 exists an optimal radius threshold for which the average rate be74 comes maximum. The performance of FFR and soft frequency 75 reuse (SFR) has been studied in [12] under both fully loaded 76 and partially loaded scenarios. An algorithm was proposed 77 in [13] for enhancing the network capacity and the cell-edge 78 performance for a dynamic SFR deployment relying on re79 alistic irregularly shaped cells. A fuzzy logic based generic 80 model was proposed for deriving different frequency reuse 81 schemes in [14]. As a further development, an FFR based 3-cell 82 network-MIMO based tri-sector BS architecture was presented 83 in [15]. FFR and SFR are compared in the presence of corre84 lated interferers in [16]. The optimal configuration of FFR is 85 determined in [17] for a high-density wireless cellular network. 86 The authors of [18] have proposed a distributed and adaptive 87 solution for interference coordination based on the center of 88 gravity of users in each sector. An optimal FFR and power 89 control scheme which can coordinate the interference among 90 the heterogeneous nodes is proposed in [19].

91 An analytical framework of calculating both the coverage 92 probability $\left(\mathrm{CP}_{r}\right)$ and the average rate of FFR schemes was 93 presented in [10] and [11] for homogeneous single input single 94 output (SISO) and MIMO heterogeneous networks, respec95 tively, using a Poisson point process (PPP). However, the au96 thors of [10], [11] assumed having an unplanned FFR network, 97 where the cells using the same frequency set are randomly 98 allocated. Hence, two cells using the same frequency for the 99 cell-edge users may in fact be co-located [10], [11]. However, 100 in case of FFR based deployments the regions using the same 101 frequency are typically planned to be as far apart as possible 102 and our focus is on these types of deployments. An FFR-aided 103 distributed antenna system (DAS) and an FFR-aided picocell 104 was studied in [20] and [21]. While, an FFR-aided femtocell 105 has been extensively studied in [22]-[26].

106 However, most of the work based on FFR has considered the 107 conventional SISO case. To the best of our knowledge, no prior 108 work has analytically derived the optimal SINR threshold for 109 FFR, when the number of antennas is high at the transmitter $110 \mathrm{and} /$ or at the receiver. Hence, in this work, we derive both the $111 \mathrm{CP}_{r}$ and the average achievable rate expressions of FFR in the 112 presence of both MU-MIMO as well as of SIMO systems and 113 derive the optimal SINR threshold corresponding to the desired $114 \mathrm{CP}_{r}$ and throughput. Furthermore, the performance of FFR115 aided MU-MIMOs is compared to that of FFR in the presence 116 of a SIMO system.

117 The key benefit of MU-MIMO is their ability to improve 118 the spectral efficiency, which has been extensively studied in 119 a single-cell context in the presence of AWGN [27]-[29]. 120 However, it has been shown in [30], [31] with the help of 121 simulation, that the efficiency of MU-MIMOs is significantly 122 eroded in a multi-cell environment due to interference, es123 pecially in the cell-edge region. FFR is capable of signifi124 cantly improving the cell-edge coverage since it uses FR3 for 125 the cell-edge users. Hence we study FFR-aided MU-MIMOs and quantify their average throughput as well as coverage 126 probability.

Furthermore, we carefully examine the correlation of the sub- 128 bands $F_{0}, F_{1}, F_{2}$ and $F_{3}$ in Fig. 1 used in the FFR system 129 considered. All prior work on FFR has assumed that the sub- 130 bands experience independent fading, which is mathematically 131 convenient, but practically not realisable. Indeed, when we 132 consider practical transmission block based modulation such as 133 OFDM, the channel's delay spread is assumed to be confined to 134 the cyclic prefix of the OFDM symbol. Such a limited-duration 135 (typically less than $20 \%$ of the useful OFDM symbol duration) 136 impulse response will result in correlation amongst the adjacent 137 frequency domain OFDM sub-channels. More explicitly, unless 138 the sub-bands $F_{0} \cdots F_{3}$ are spaced apart by more than the recip- 139 rocal of the delay spread, correlation will exist. Since the delay 140 spread experienced in the downlink is user-dependent, it is vir- 141 tually impossible to ensure that the sub-bands $F_{i}$ in Fig. 1 are in- 142 dependent for each user scheduled in the downlink. Therefore, 143 in our analysis we will specifically take into account the corre- 144 lation of the sub-bands. For FFR-aided MU-MIMO and SIMO 145 systems, the expressions of $\mathrm{CP}_{r}$ and average rate are derived 146 and the following new results are presented:

(a) The optimal SINR threshold that maximizes the $\mathrm{CP}_{r}$ of 148 FFR is derived for a given $T$. We show that the optimal 149 $S_{t h}$ (denoted by $S_{o p t, C}$ ) is $S_{t h}=T$ for both the MU-MIMO 150 and SIMO system, and if we choose the SINR threshold 151 to be $S_{\text {opt }, C}$, then the achievable $\mathrm{CP}_{r}$ of FFR is higher 152 than that of FR3. The improvement of the FFR $\mathrm{CP}_{r}$ over 153 that of FR3 is due to the resultant sub-band diversity gain 154 achieved by the systems when a user is classified as either 155 a cell-centre or a cell-edge user.

(b) The optimal SINR threshold that maximizes the average 157 rate of FFR is derived. We show that the optimal $S_{t h}$ (de- 158 noted by $S_{o p t, R}$ ) is equal to $T^{\prime}$ for both MU-MIMO and 159 SIMO systems, where $T^{\prime}$ is a fixed SINR value, which de- 160 pends on the system parameters such as the path loss expo- 161 nent, the number of antennas, the fading parameters, etc. 162

(c) The correlation of the sub-bands always degrades both the 163 $\mathrm{CP}_{r}$ and the average rate of the FFR-aided MU-MIMO 164 and SIMO systems.

165

(d) The performance of FFR-aided MU-MIMO and SIMO 166 systems is compared. It is shown that system designer 167 may choose the $(2 \times 2)$ MU-MIMO system over $(1 \times 3) 168$ SIMO system of FFR scheme as MU-MIMO achieves 169 significant gain in average rate over SIMO.

We will demonstrate that our analytical results are in close 171 agreement with the simulation results. Moreover, it is shown 172 that at optimal $S_{t h}$, the FFR achieves significantly high gain in 173 $\mathrm{CP}_{r}$ than that of average rate with respect to FR1 and hence this 174 scheme would be more useful when coverage gain is essentially 175 required. Therefore, FFR-aided MU-MIMO provides both high 176 average rate and satisfactory $\mathrm{CP}_{r}$ for a lower value of $N_{a}$.

\section{SYSTEM MODEL}

A homogeneous macrocell network relying on hexagonal 179 tessellation and on an inter cell site distance of $2 R$ is considered, 180 


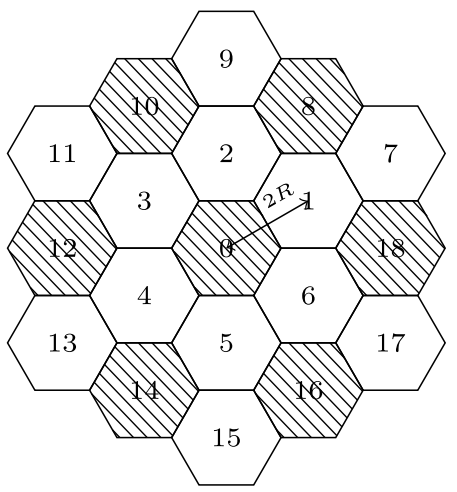

Fig. 2. Hexagonal structure of 2-tier macrocell. Interference for 0th cell in FR1 system is contributed form all the neighbouring 18 cells, while in a FR3 system it is contributed only from the shaded cells.

181 as shown in Fig. 2. Both a MU-MIMO and a SIMO system is 182 considered. We assume that in the MU-MIMO case each user 183 is equipped with $N_{r}$ receive antennas, while the BS is equipped 184 with $N_{t}$ transmit antennas and that $N_{t}=N_{r}$. Our focus is on the 185 downlink and hence $N_{t}$ transmit antennas are used for transmis186 sion, while the $N_{r}$ receive antennas at the UE are used for re187 ception. We also assume that all $N_{t}$ transmit antennas at the BS 188 are utilized to transmit $N_{t}$ independent data steams to its own $N_{t}$ 189 users. A linear minimum mean-square-error (LMMSE) receiver 190 [32] is considered. In order to calculate the post-processing 191 SINR of this LMMSE receiver, it is assumed that the $\left(N_{r}-1\right)$ 192 closest interferers can be completely cancelled using the anten193 nas at the receiver. ${ }^{3}$ For example, in the MU-MIMO case, the 194 user will not experience any intra-tier interference emanating 195 from the serving BS as $N_{t}=N_{r}$. In the SIMO case each user 196 is equipped with $N_{r}$ antennas. The SINR $\eta_{t}(r)$ of a user in the 197 MU-MIMO system and the SINR $\eta_{r}(r)$ of a user in the SIMO 198 system located at $r$ meters from its serving BS are given by

$$
\eta_{t}(r)=\frac{g r^{-\alpha}}{\frac{\sigma^{2}}{P}+I_{t}}, \quad I_{t}=\sum_{i \in \psi} \sum_{j=1}^{N_{t}} h_{i j} d_{i}^{-\alpha}
$$

199 and

$$
\eta_{r}(r)=\frac{g r^{-\alpha}}{\frac{\sigma^{2}}{P}+I_{r}}, \quad I_{r}=\sum_{i \in \psi_{r}} h_{i j} d_{i}^{-\alpha},
$$

200 respectively, where the transmit power of a BS is denoted by $P$. 201 Here $\psi$ is the set of interfering BSs in the FR1 network and $\psi_{r}$ 202 denotes all the interfering BSs, excluding the nearest $\left(N_{r}-1\right)$ 203 interferers, while $N_{t}$ denotes the number of transmit antennas. 204 The standard path loss model of $\|x\|^{-\alpha}$ is assumed, where $205 \alpha \geq 2$ is the path loss exponent and $\|x\|$ is the distance of a user 206 from the BS. We assumed that the users are at least at a distance 207 of $d$ away from the BS. ${ }^{4}$ The noise power is denoted by $\sigma^{2}$. 208 Here, $r$ and $d_{i}$ are the distances from the user to the serving BS 209 and to the $i^{\text {th }}$ interfering BS, respectively, while $g$ and $h_{i}$ denote

\footnotetext{
${ }^{3}$ It is widely exploited that using the LMMSE receiver $\left(N_{r}-1\right)$ interferers can be mitigated, where $N_{r}$ is the number of receive antennas [32]. However, for simplicity, we assume that the $N_{r}-1$ closest interferers can be completely cancelled.

${ }^{4}$ Typically, the path loss model is assumed to be $\max \{d,\|x\|\}^{-\alpha}$.
}

the corresponding channel fading power, which are independent 210 and identically exponentially distributed (i.i.d.) with a unit 211 mean, i.e., $g \sim \exp (1)$ and $h_{i} \sim \exp (1) \forall i$. In MU-MIMO case, 212 $h_{i j}$ is the channel's fading power from the $j^{\text {th }}$ antenna of the 213 $i^{t h}$ interfering BS to the user and it is i.i.d. with a unit mean. 214 Without loss of generality we have considered a user in the $0^{\text {th }} 215$ cell of Fig. 2 in our analysis.

Similar to [10], the subscribers are classified as cell-centre 217 users and cell-edge users based on the SINR at the mobile sta- 218 tion. If the calculated SINR of a user is lower than the specified 219 SINR threshold $S_{t h}$, the user is classified as a cell-edge user. 220 Otherwise, the user is classified as a cell-centre user. Typically, 221 FFR divides the whole frequency band into a total of $(1+\delta) 222$ parts, where $F_{0}$ is allocated to all the cells for the cell-centre 223 users, as seen in Fig. 1. One of the $\{1, \cdots, \delta\}$ parts is assigned 224 to the cell-edge users in each cell in a planned fashion. The 225 users are assumed to be uniformly distributed in a cell and all re- 226 source blocks are uniformly shared among the users. The trans- 227 mit power is assumed to be fixed. If we have $\eta_{t}(r)\left(\right.$ or $\left.\eta_{r}(r)\right) \geq 228$ $S_{t h}$ for a user, then the user will continue to experience the same 229 fading power, i.e., $g$ and $h_{i}$ from the user to the serving BS 230 and to the $i^{\text {th }}$ interfering BS, respectively. However, if we have 231 $\eta_{t}(r)$ (or $\left.\eta_{r}(r)\right)<S_{t h}$ for a user, the user is allocated another 232 sub-band (from the set of sub-bands assigned to cell-edge users) 233 and it experiences a new fading power, i.e., $\hat{g}$ and $\hat{h}_{i}$ from the 234 user to the serving BS and to the $i^{\text {th }}$ interfering BS, respectively. 235 Based on the coherence bandwidth of the OFDM system, and 236 the bands associated with $F_{0}$ to $F_{3}$ in Fig. 1 is is possible that $\hat{g} 237$ and $\hat{h}_{i}$ are either correlated with or independent of $g$ and $h_{i}$, re- 238 spectively. Note that $g, \hat{g}, h_{i}$, and $\hat{h}_{i}$ are the channel gains in the 239 frequency domain and the term correlation is used for referring 240 to frequency domain correlation in this paper. The correlation 241 depends both on the particular user's channel conditions and 242 on the instantaneous coherence bandwidth with respect to the 243 FFR frequency bands. To better understand the impact of corre- 244 lation among the sub-bands on the FFR system's performance, 245 in this paper, we consider the following two extreme cases: 246

Case 1: $g$ and $\hat{g}$ are independent and also $h_{i}$ as well as $\hat{h}_{i}$, are 247 independent for all $i$.

248

Case 2: $g$ and $\hat{g}$ are fully correlated and also $h_{i}$ as well as $\hat{h}_{i}, 249$ are fully correlated for all $i$.

In reality these channel output powers may be partially corre- 251 lated, but the analysis of partial (arbitrary) correlation is quite 252 complicated and hence it is beyond the scope of this work. 253 However, the analysis of the above two extreme cases we be- 254 lieve, is sufficient for understanding the impact of correlation 255 among the sub-bands.

\section{Coverage Probability Analysis of FFR}

In this section, we first derive the $\mathrm{CP}_{r}$ of both the 258 MU-MIMO and SIMO system considered, which is defined 259 as the probability that a randomly chosen user's instantaneous 260 SINR $\eta_{t}(r)$ is higher than $T$. This defines, the average fraction 261 of users are having an SINR higher than the target SINR. The 262 coverage probability is determined by the complementry cumu- 263 lative distribution function of the SINR over the network. The 264 
$265 \mathrm{CP}_{r}$ of a user who is at a distance of $r$ meters from the $\mathrm{BS}$ in a 266 FR1-aided MU-MIMO scenario is given by

$$
P_{1}(T, r)=P\left[\eta_{t}(r)>T\right]=P\left[g>\operatorname{Tr}^{\alpha} I_{t}+\operatorname{Tr}^{\alpha} \frac{\sigma^{2}}{P}\right],
$$

267 where $I_{t}$ is defined in (2). Since $g \sim \exp (1), h_{i j} \sim \exp (1)$, and $268 h_{i j}$ are i.i.d., $P_{1}(T, r)$ is given by

$$
\begin{gathered}
P_{1}(T, r)=E_{h_{i j}}\left[e^{-\operatorname{Tr}^{\alpha} I_{t}-T^{\alpha} \frac{\sigma^{2}}{P}}\right]=\prod_{i \in \psi} \prod_{j=1}^{N_{t}} E_{h_{i j}}\left[e^{-T^{\alpha} h_{i j} d_{i}^{-\alpha}}\right] \\
\times e^{-T^{\alpha} \frac{\sigma^{2}}{P}}=\prod_{i \in \psi}\left(\frac{1}{1+\operatorname{Tr}^{\alpha} d_{i}^{-\alpha}}\right)^{N_{t}} e^{-\operatorname{Tr}^{\alpha} \frac{\sigma^{2}}{P}}
\end{gathered}
$$

269 where $\psi$ is the set of interfering BSs in a FR1 network. 270 Similarly, the $\mathrm{CP}_{r}$ of a user located at a distance of $r$ meters 271 from the BS in a FR3 network can be formulated as

$$
P_{3}(T, r)=\prod_{i \in \phi}\left(\frac{1}{1+T r^{\alpha} d_{i}^{-\alpha}}\right)^{N_{t}} e^{-T r^{\alpha} \frac{\sigma^{2}}{P}}
$$

272 where $\phi$ is the set of interfering cells in the FR3 scheme, which 273 is a function of the frequency reuse plan. Also, the $\mathrm{CP}_{r}$ of a user 274 in the SIMO-based FR1 network and in a FR3 network can be 275 expressed as

$$
\begin{aligned}
& P_{1}(T, r)=\prod_{i \in \psi_{r}} \frac{1}{1+T r^{\alpha} d_{i}^{-\alpha}} e^{-T r^{\alpha} \frac{\sigma^{2}}{P}} \quad \text { and } \\
& P_{3}(T, r)=\prod_{i \in \phi_{r}} \frac{1}{1+T r^{\alpha} d_{i}^{-\alpha}} e^{-T r^{\alpha} \frac{\sigma^{2}}{P}} .
\end{aligned}
$$

276 Here $\phi_{r}$ denotes the set of interfering cells in the FR3 scheme 277 excluding the nearest $\left(N_{r}-1\right)$ interferers. Let us now derive 278 the $\mathrm{CP}_{r}$ of FFR for both the independent and correlated cases.

279 A. Case 1: $g$ and $\hat{g}$ are Independent as Well as $h_{i}$ and $\hat{h}_{i}$ are 280 Also Independent for all $i$

281 The $\mathrm{CP}_{r} P_{F, c}(r)$ of a cell-centre user who is at a distance of $282 r$ meters from the $0^{\text {th }}$ BS in a FFR-aided MU-MIMO scenario 283 is given by

$$
\begin{aligned}
P_{F, c}(r) & \stackrel{(a)}{=} P\left[\eta_{t}(r)>T \mid \eta_{t}(r)>S_{t h}\right] \\
& =P\left[\frac{g r^{-\alpha}}{I_{t}+\frac{\sigma^{2}}{P}}>T \mid \frac{g r^{-\alpha}}{I_{t}+\frac{\sigma^{2}}{P}}>S_{t h}\right],
\end{aligned}
$$

284 where $(a)$ follows from the fact that a cell-centre user has SINR $285 \geq S_{t h}$. Upon applying Bayes' rule, one can rewrite $P_{F, c}(r)$ as

$$
\begin{aligned}
P_{F, c}(r) & =\frac{P\left[\frac{g r^{-\alpha}}{I_{t}+\frac{\sigma^{2}}{P}}>T, \frac{g r^{-\alpha}}{I_{t}+\frac{\sigma^{2}}{P}}>S_{t h}\right]}{P\left[\frac{g r^{-\alpha}}{I_{t}+\frac{\sigma^{2}}{P}}>S_{t h}\right]} \\
= & \frac{\prod_{i \in \psi}\left(\frac{1}{1+\max \left\{T, S_{t h}\right\} r^{\alpha} d_{i}^{-\alpha}}\right)^{N_{t}} e^{-\max \left\{T, S_{t h}\right\} r^{\alpha} \frac{\sigma^{2}}{P}}}{\prod_{j \in \psi}\left(\frac{1}{1+S_{t h} r^{\alpha} d_{j}^{-\alpha}}\right)^{N_{t}} e^{-S_{t h} r^{\alpha} \frac{\sigma^{2}}{P}}} .
\end{aligned}
$$

Similarly, the $\mathrm{CP}_{r}$ of a cell-edge user who is at a distance of $r 286$ meters from the BS in the FFR-aided MU-MIMO case $P_{F, e}(r) 287$ is given by

$$
\begin{aligned}
P_{F, e}(r) & =P\left[\hat{\eta}_{t}(r)>T \mid \eta_{t}(r)<S_{t h}\right] \\
& =\frac{P\left[\frac{\hat{g} r^{-\alpha}}{\hat{I}_{t}+\frac{\sigma^{2}}{P}}>T, \frac{g r^{-\alpha}}{I_{t}+\frac{\sigma^{2}}{P}}<S_{t h}\right]}{P\left[\frac{g r^{-\alpha}}{I_{t}+\frac{\sigma^{2}}{P}}<S_{t h}\right]} .
\end{aligned}
$$

Here, the cell-edge user will experience the new interference 289 term of $\hat{I}_{t}=\sum_{i \in \phi} \sum_{j=1}^{N_{t}} \hat{h}_{i j} d_{i}^{-\alpha}$ and the new channel power $\hat{g}$, i.e. a 290 new SINR $\hat{\eta}(r)$ due to the fact that the cell-edge user is now a 291 FR3 user. Basically, $\hat{\eta}(r)$ denotes the SINR experienced by the 292 user at a distance of $r$ meters from the BS in a FR3 system and 293 is given by

$$
\hat{\eta}(r)=\frac{\hat{g} r^{-\alpha}}{\hat{I}_{t}+\frac{\sigma^{2}}{P}}, \quad \hat{I}_{t}=\sum_{i \in \phi} \sum_{j=1}^{N_{t}} \hat{h}_{i j} d_{i}^{-\alpha} .
$$

Since both $g$ and $\hat{g}$ as well as $h_{i}$ and $\hat{h}_{i}$ are assumed to be i.i.d, 295 $P_{F, e}(r)$ can be simplified to

$$
P_{F, e}(r)=P\left[\frac{\hat{g} r^{-\alpha}}{\hat{I}_{t}+\frac{\sigma^{2}}{P}}>T\right]=P_{3}(T, r) .
$$

Let us now derive the $\mathrm{CP}_{r} P_{f}(r)$ of a user in the FFR-aided 297 MU-MIMO system, which can be written as

$$
P_{F}(r)=P_{F, c}(r) P\left[\eta_{t}(r)>S_{t h}\right]+P_{F, e}(r) P\left[\eta_{t}(r)<S_{t h}\right] .
$$

Here, the first term denotes the $\mathrm{CP}_{r}$ contributed by the cell- 299 centre users, while the second term denotes the contribution of 300 the cell-edge users. By using the expression in (7) for $P_{F, c}(r) 301$ and the expression in (9) for $P_{F, e}(r),(10)$ can be simpli- 302 fied to

$$
\begin{array}{r}
P_{F}(r)=\prod_{i \in \psi}\left(\frac{1}{1+\max \left\{T, S_{t h}\right\} r^{\alpha} d_{i}^{-\alpha}}\right)^{N_{t}} e^{-\max \left\{T, S_{t h}\right\} r^{\alpha} \frac{\sigma^{2}}{P}} \\
+P_{3}(T, r)-P_{3}(T, r) P_{1}\left(S_{t h}, r\right) .
\end{array}
$$

Lemma 1: The optimum $S_{\text {th }}$ (denoted by $S_{\text {opt }, C}$ ) that maxi- 304 mizes the FFR-aided coverage probability is $S_{t h}=T$, and when 305 the SINR threshold is set to $S_{\text {opt }, c}$, the coverage probability of 306 FFR becomes higher than that of FR3.

Proof: See Appendix A for the proof.

B. Case 2: $g$ and $\hat{g}$ are Completely Correlated as Well as $h_{i} \quad 309$ and $\hat{h}_{i}$ are Also Completely Correlated for all $i$

Note that the centre $\mathrm{CP}_{r}$ is the same for both the above 311 Case 1 and for this case, since a user does not change its sub- 312 band, when it becomes a cell-centre user because if $\eta_{t}(r) \geq S_{t h} 313$ for a user, then it will continue to experience the same fading 314 power. However, the edge $\mathrm{CP}_{r}$ is different in Case 1 as well as 315 Case 2, and in this scenario the $\mathrm{CP}_{r} P_{F, e}(r)$ of a cell-edge user, 316 
317 who is at a distance of $r$ meters from the BS in our FFR network 318 is given by

$$
P_{F, e}(r)=P\left[\hat{\eta}_{t}(r)>T \mid \eta_{t}(r)<S_{t h}\right]=\frac{P\left[\hat{\eta}_{t}(r)>T, \eta_{t}(r)<S_{t h}\right]}{P\left[\eta_{t}(r)<S_{t h}\right]} .
$$

319 Substituting the value of $P_{F, c}$ and $P_{F, e}$ from (7) and (12) into 320 Eq. (10), the $\mathrm{CP}_{r} P_{f}(r)$ in our FFR network can be written as

$$
\begin{array}{r}
P_{F}(r)=\prod_{i \in \psi}\left(\frac{1}{1+\max \left\{T, S_{t h}\right\} r^{\alpha} d_{i}^{-\alpha}}\right)^{N_{t}} e^{-\max \left\{T, S_{t h}\right\} r^{\alpha} \frac{\sigma^{2}}{P}} \\
+P\left[\hat{\eta}_{t}(r)>T, \eta_{t}(r)<S_{t h}\right] .
\end{array}
$$

321 Recall that $\eta_{t}(r)$ and $\hat{\eta}_{t}(r)$ represent the SINR experienced by a 322 user in an FR1 and an FR3 system, respectively. Note that even 323 though $g$ and $\hat{g}$ as well as $h_{i}$ and $\hat{h}_{i}$ are completely correlated, $324 \eta_{t}(r)$ is not the same as $\hat{\eta}_{t}(r)$, because the set of interferers are 325 different in the denominator of the $\eta_{t}(r)$ and $\hat{\eta}_{t}(r)$ expressions 326 given in (2) and (8), respectively, i.e., $\psi$ corresponds to the 327 set of interferers in the FR1 network, while $\phi$ corresponds to 328 the set of interferers in the FR3 network. Since $g$ and $\hat{g}$ are 329 completely correlated and $h_{i}$ and $\hat{h}_{i}$ are also completely corre330 lated for all $i$, we use the following transformation to further 331 simplify $P_{F}(r)$ :

$$
P\left[\hat{\eta}_{t}(r)>T, \eta_{t}(r)<S_{t h}\right]=P\left[\hat{\eta}_{t}(r)>T, \hat{\eta}_{t}(r)<\hat{S}_{t h}\right] .
$$

332 Basically instead of marking a user as a cell-edge user based 333 on the FR1 SINR $\eta_{t}(r)$, we mark them on the basis of the FR3 334 SINR $\hat{\eta}_{t}(r)$ by introducing a new SINR threshold $\hat{S}_{t h}$. In other 335 words, we introduce a new SINR threshold $\hat{S}_{t h}$ for ensuring that 336 if for any user we have $\eta_{t}(r)<S_{t h}$, then for the same user we 337 have $\hat{\eta}_{t}(r)<\hat{S}_{t h}$ and vice-versa. The threshold $\hat{S}_{t h}$ is computed 338 using the relationship of $P\left[\eta_{t}(r)<S_{t h}\right]=P\left[\hat{\eta}_{t}(r)<\hat{S}_{t h}\right]$. This 339 ensures that the same user is marked as a cell-edge user for both 340 reuse patterns FR1 and FR3. Now, using the transformation 341 given in $(14), P_{F}(r)$ can be simplified to

$$
\begin{aligned}
P_{F}(r)=\prod_{i \in \psi} & \left(\frac{1}{1+\max \left\{T, S_{t h}\right\} r^{\alpha} d_{i}^{-\alpha}}\right)^{N_{t}} e^{-\max \left\{T, S_{t h}\right\} r^{\alpha} \frac{\sigma^{2}}{P}} \\
& +P[\hat{\eta}(r)>T]-P\left[\hat{\eta}(r)>\max \left\{\hat{S}_{t h}, T\right\}\right] .
\end{aligned}
$$

342 In this case, to obtain the optimum $S_{\text {opt }, C}$, we consider the 343 following two possibilities: (i) $S_{t h} \geq T$, (ii) $S_{t h}<T$.

(i) $S_{t h} \geq T$ : In this scenario, $C P_{f}(r)$ can be expressed in terms of $T$ as:

$$
\begin{array}{r}
P_{F}\left(r, S_{t h} \geq T\right)=\prod_{i \in \psi} \frac{1}{1+} S_{t h} r^{\alpha} d_{i}^{-\alpha} e^{-S_{t h} r^{\alpha} \frac{\sigma^{2}}{P}} \\
+P_{3}(T, r)-P_{3}\left(\hat{S}_{t h}, r\right) .
\end{array}
$$

346 Since we have $P_{3}\left(\hat{S}_{t h}, r\right)=P_{1}\left(S_{t h}, r\right)$ and $P_{1}\left(S_{t h}, r\right)=$ 347

$$
\begin{aligned}
& \prod_{i \in \psi}\left(\frac{1}{1+S_{t h} r^{\alpha} d_{i}^{-\alpha}}\right)^{N_{t}} e^{-S_{t h} r^{\alpha} \frac{\sigma^{2}}{P}} \text {, hence } \\
& P_{F}\left(r, S_{t h} \geq T\right)=P_{3}(T, r) .
\end{aligned}
$$

(ii) $S_{t h}<T$ : In this case $P_{f}(r)$ can be formulated in terms 348 of $T$ as:

$$
\begin{aligned}
P_{F}\left(r, S_{t h}<T\right) & =\prod_{i \in \psi}\left(\frac{1}{1+\operatorname{Tr}^{\alpha} d_{i}^{-\alpha}}\right)^{N_{t}} e^{-T^{\alpha} \frac{\sigma^{2}}{P}} \\
& +P_{3}(T, r)-P_{3}\left(\max \left\{\hat{S}_{t h}, T\right\}, r\right) .
\end{aligned}
$$

Note that when $S_{t h}<T, \hat{S}_{t h}$ may be higher or lower than $T .350$ When $\hat{S}_{t h}>T$,

$$
P_{3}\left(\max \left\{\hat{S}_{t h}, T\right\}, r\right)=P_{3}\left(\hat{S}_{t h}, r\right)=P_{1}\left(S_{t h}, r\right)>P_{1}(T, r)
$$

since $S_{t h}<T$. And when $\hat{S}_{t h}<T$, we have:

$$
P_{3}\left(\max \left\{\hat{S}_{t h}, T\right\}, r\right)=P_{3}(T, r)>P_{1}(T, r) .
$$

Hence, we arrive at:

$$
\begin{aligned}
P_{F}\left(r, S_{t h}\right. & <T)=\prod_{i \in \psi}\left(\frac{1}{1+\operatorname{Tr}^{\alpha} d_{i}^{-\alpha}}\right)^{N_{t}} e^{-\operatorname{Tr}^{\alpha} \frac{\sigma^{2}}{P}} \\
& +P_{3}(T, r)-P_{3}\left(\max \left\{\hat{S}_{t h}, T\right\}, r\right)<P_{3}(T, r) .
\end{aligned}
$$

Comparing the FFR $\mathrm{CP}_{r}$ for $S_{t h} \geq T$ and $S_{t h}<T$ given by (17) 354 and (21), respectively, it becomes apparent that $P_{F}\left(r, S_{t h} \geq 355\right.$ $T)>P_{F}\left(r, S_{t h}<T\right)$. In other words, when the fading is fully 356 correlated across the sub-bands, the optimal choice of the SINR 357 threshold is $S_{t h} \geq T$ and at the optimal SINR threshold the FFR 358 scheme succeeds in achieving the $\mathrm{FR} 3 \mathrm{CP}_{r}$. Unlike for Case 1, 359 the FFR $\mathrm{CP}_{r}$ is not better than the $\mathrm{FR} 3 \mathrm{CP}_{r}$ since there is no sub- 360 band diversity gain, when a user moves from the cell-centre to 361 the cell-edge region.

362

In order to find the $\mathrm{CP}_{r}$ for a typical user, we have to calculate 363 the probability density function (pdf) of $r$, which is the distance 364 between the $0^{\text {th }}$ BS (serving BS) and the desired user. To 365 calculate this pdf, we model the cell shape by an inner circle 366 within a hexagonal cell [33], and assume that the users are 367 uniformly distributed. Therefore, the pdf $f_{R}(r)$ of $r$ is given by 368

$$
\begin{aligned}
& f_{R}(r)=\left\{\begin{array}{c}
\frac{2 r}{R^{2}}, r \leqslant R \\
0, r>R
\end{array}\right. \\
& \text { IV. AVERAGE RATE }
\end{aligned}
$$

In this section, we derive the average rate of both the FFR- 370 aided MU-MIMO as well as of its SIMO counterpart and find 371 the optimum value of $S_{t h}$ (denoted by $S_{\text {opt }, R}$ ) for which the 372 average rate is maximum. The average rate of the system is 373 given by $R=E[\ln (1+\mathrm{SINR})]$. In order to derive the average 374 rate $^{5}$ for the FFR system, we have to consider its sub-band al- 375 location. Since the users are uniformly distributed, the specific 376 sub-band allocated to the cell-centre users and cell-edge users 377 are given by [9], [10] $N_{c}=N_{t} P_{F, c}$ and $N_{e}=\frac{N_{t}-N_{c}}{3}$, where $P_{F, c} 378$ denotes the specific fraction of cell-centre users, while $N_{t}, N_{c} 379$ and $N_{e}$ denote the total band, cell-centre sub-band and cell-edge 380

\footnotetext{
${ }^{5}$ An interference limited system is assumed for simplicity, which implies ignoring the effects of noise. However, the derivation of the average rate can be readily extended to the case, where the thermal noise is also considered.
} 
381 sub-band, respectively. Let us now derive the average rate for 382 the planned FFR-aided MU-MIMO case.

\section{A. Average Rate in the FRI and FR3 Systems}

384 The average rate of a user at a distance $r$ is $E\left[\ln \left(1+\eta_{t}(r)\right)\right]$. 385 By exploiting the fact that for a positive random variable $X=$ $386 \ln \left(1+\eta_{t}(r)\right)$ we have $E[X]=\int_{t>0} P(X>t) \mathrm{d} t$, the rate $R_{1}(r)$ 387 can be rewritten as

$$
\begin{aligned}
R_{1}(r) & =\int_{t>0} P\left[\ln \left(1+\eta_{t}(r)\right)>t\right] \mathrm{d} t=\int_{t>0} P\left[\eta_{t}(r)>e^{t}-1\right] \mathrm{d} t \\
& =\int_{t>0} \prod_{j \in \psi}\left(\frac{1}{1+\left(e^{t}-1\right) r^{\alpha} d_{j}^{-\alpha}}\right)^{N_{t}} \mathrm{~d} t
\end{aligned}
$$

388 which follows from (3) and (4). Let us now determine the 389 average rate of the FR1 system, where spatially averaged rate $390 R_{1}$ can be expressed as

$$
R_{1}=\int_{0}^{R} \int_{t>0} \prod_{j \in \psi}\left(\frac{1}{1+\left(e^{t}-1\right) r^{\alpha} d_{j}^{-\alpha}}\right)^{N_{t}} \mathrm{~d} t f_{R}(r) \mathrm{d} r .
$$

391 The average rate of FR3 can be obtained in a similar fashion, 392 which is given by

$$
R_{3}=\int_{0}^{R} \int_{t>0} \prod_{i \in \phi}\left(\frac{1}{1+\left(e^{t}-1\right) r^{\alpha} d_{i}^{-\alpha}}\right)^{N_{t}} \mathrm{~d} t f_{R}(r) \mathrm{d} r .
$$

393 B. Average Rate of the FFR System, When the $394 \mathrm{Sub}$-Bands are Independent

395 Lemma 2: The average rate of the FFR-aided MU-MIMO 396 system is given by

$$
\begin{aligned}
R_{f}=\int_{0}^{R} \int_{t>0}( & \prod_{j \in \psi}\left(\frac{1}{1+\max \left\{e^{t}-1, S_{t h}\right\} r^{\alpha} d_{j}^{-\alpha}}\right)^{N_{t}} \\
& \left.+\frac{1}{3} \prod_{i \in \phi} \frac{P\left[\eta_{t}(r)<S_{t h}\right]}{\left(1+\left(e^{t}-1\right) r^{\alpha} d_{i}^{-\alpha}\right)^{N_{t}}}\right) \mathrm{d} t f_{R}(r) \mathrm{d} r .
\end{aligned}
$$

Proof: See Appendix B for the proof.

Similarly, the average rate of the FFR-aided SIMO system is 398 given by

$$
\begin{aligned}
R_{f}=\int_{0}^{R} \int_{t>0}\left(\prod_{j \in \psi_{r}}\right. & \frac{1}{1+\max \left\{e^{t}-1, S_{t h}\right\} r^{\alpha} d_{j}^{-\alpha}} \\
& \left.+\frac{1}{3} \prod_{i \in \phi_{r}} \frac{P\left[\eta_{r}(r)<S_{t h}\right]}{1+\left(e^{t}-1\right) r^{\alpha} d_{i}^{-\alpha}}\right) \mathrm{d} t f_{R}(r) \mathrm{d} r
\end{aligned}
$$

\section{Optimum Value of the SIR Threshold $S_{\text {opt }, R}$, When the Sub-Bands are Independent} 400

The optimum value of $S_{t h}$ (denoted by $S_{o p t, R}$ ) for which the 402 average rate of the FFR system is maximized is derived and it 403 is shown to be a function of both the number of antennas and of 404 the path loss exponent.

405

Lemma 3: The value of $S_{t h}$ which maximizes the average rate 406 of the FFR system is $S_{o p t, R}=T^{\prime}$, where $T^{\prime}$ can be obtained as 407 the solution of equation given in (28), shown at the bottom of 408 the page, where, $K(r)$ is defined later in (47).

409

Proof: See Appendix C for the proof.

Note that the optimal $S_{t h}$ of the SIMO scenario can be derived 411 by following the method of the MU-MIMO case and it is 412 $S_{\text {opt }, R}=T^{\prime}$, where $T^{\prime}$ can be obtained as the solution of the 413 equation given in (29), shown at the bottom of the page, where 414 we have $K(r)=\frac{1}{3} \int \prod_{t>0} \frac{1}{1+\left(e^{t}-1\right) r^{\alpha} d_{i}^{-\alpha}} \mathrm{d} t$.

Fig. 3 plots the optimal SINR threshold $S_{t h}$ versus the number 416 of antennas for different path loss exponent. It can be observed 417 for the MU-MIMO case that as the number of transmit antennas 418 is reduced, $S_{\text {opt }, R}$ increases. Intuitively, as the number of trans- 419 mit antennas decreases, the interference experienced by the user 420 would decrease as the interference from the other cell decrease. 421 Thus, the average SINR of all users increases. Hence, the opti- 422 mal SINR threshold increases in order to balance the ratio of 423 cell-edge users and cell-centre users. Similarly, as the number 424 of receive antennas increases, the average SINR increases in 425 SIMO scenario, because more antennas are capable of can- 426 celling more of the closest interferers. Hence, $S_{o p t, R}$ increases 427

$$
\int_{0}^{R}\left(\frac{\left(K(r)-\ln \left(1+T^{\prime}\right)\right) \sum_{i \in \psi}\left(1+T^{\prime} r^{\alpha} d_{i}^{-\alpha}\right)^{N_{t}-1} r^{\alpha} d_{i}^{-\alpha}\left(\prod_{j \in \psi \backslash i}\left(1+T^{\prime} r^{\alpha} d_{j}^{-\alpha}\right)^{N_{t}}\right)}{\left(\prod_{j \in \psi}\left(1+T^{\prime} r^{\alpha} d_{j}^{-\alpha}\right)\right)^{2 N_{t}}}\right) f_{R}(r) \mathrm{d} r=0,
$$

$$
\int_{0}^{R}\left(\frac{\left(K(r)-\ln \left(1+T^{\prime}\right)\right) \sum_{i \in \psi_{r}} r^{\alpha} d_{i}^{-\alpha}\left(\prod_{j \in \psi_{r} \backslash i}\left(1+T^{\prime} r^{\alpha} d_{j}^{-\alpha}\right)\right)}{\left(\prod_{j \in \psi_{r}}\left(1+T^{\prime} r^{\alpha} d_{j}^{-\alpha}\right)\right)^{2}}\right) f_{R}(r) \mathrm{d} r=0,
$$




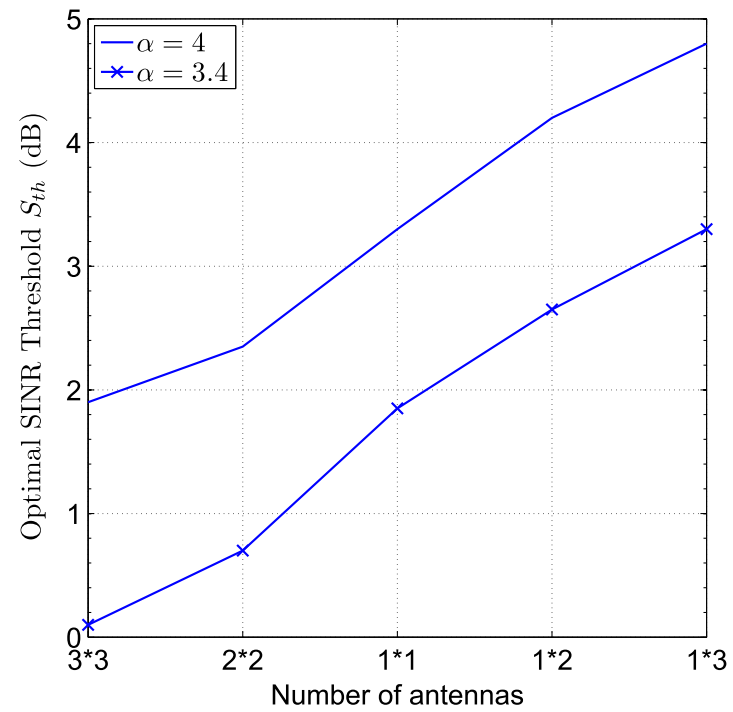

Fig. 3. Optimal SINR threshold $S_{t h}$ evaluated using (28) and (29) versus the number of antennas for different path-loss exponents.

428 in order to balance the ratio of cell-centre users and cell-edge 429 users. Furthermore, as the path loss exponent decreases, the 430 average SIR of all the users decreases and hence $S_{o p t, R}$ 431 decreases.

432 D. Average Rate of the FFR System, When the Sub-Bands are 433 Completely Correlated

434 In this subsection first we derive the average rate $R_{f}(r)$ of the 435 FFR system for the MU-MIMO case. The average rate of the 436 FFR system given in (39) can be rewritten as

$$
R_{f}(r)=R_{c}(r) P\left[\eta_{t}(r)>S_{t h}\right]+\frac{1}{3} R_{e}(r) P\left[\eta_{t}(r)<S_{t h}\right]
$$

437 Note that the first term $R_{c}(r) P\left[\eta_{t}(r)>S_{t h}\right]$ denotes the average 438 rate contributed by the cell-centre users and it is the same 439 regardless, whether the fading of the bands is correlated or inde440 pendent across the sub-bands. Similar to the average rate of the 441 FFR system given in (39), the factor $\frac{1}{3}$ is introduced in the sec442 ond term, since a frequency reuse factor of $\frac{1}{3}$ is invoked for the 443 cell-edge users. In other words, only one third of the cell-edge 444 frequency $\left(F_{1}+F_{2}+F_{3}\right)$ is used for the cell-edge users and 445 hence the factor $\frac{1}{3}$ multiplies the second term of (30). Now, us446 ing the expression of $R_{e}(r)$ in $(42), R_{e}(r) P\left[\eta_{t}(r)<S_{t h}\right]$ can be 447 written as

$$
R_{e}(r) P\left[\eta_{t}(r)<S_{t h}\right]=\int_{t>0} P\left[\hat{\eta}_{t}(r)>e^{t}-1, \eta_{t}(r)<S_{t h}\right] \mathrm{dt} .
$$

448 Using the transformation in (14), $R_{e}(r) P\left[\eta_{t}(r)<S_{t h}\right]$ can be 449 simplified to

$$
\begin{aligned}
R_{e}(r) P\left[\eta_{t}(r)<S_{t h}\right]= & \int_{t>0} P\left[\hat{\eta}_{t}(r)>e^{t}-1\right] \\
& -P\left[\hat{\eta}_{t}(r)>\max \left\{e^{t}-1, \hat{S}_{t h}\right\}\right] \mathrm{dt} .
\end{aligned}
$$

Using the result of $(25), R_{e}(r) P\left[\eta(r)<S_{t h}\right]$ can be further 450 simplified to

$$
\begin{aligned}
R_{e}(r) P\left[\eta_{t}(r)<S_{t h}\right] & =\int_{t>0} \prod_{i \in \phi} \frac{1}{1+\left(e^{t}-1\right) r^{\alpha} d_{i}^{-\alpha}} \\
& -\prod_{i \in \phi} \frac{1}{1+\max \left\{e^{t}-1, \hat{S}_{t h}\right\} r^{\alpha} d_{i}^{-\alpha}} \mathrm{dt} .
\end{aligned}
$$

Finally, substituting back (41) as well as (33) into (30) and then 452 averaging over the spatial dimension, the average rate of the 453 FFR system is given as

$$
\begin{gathered}
R_{f}=\int_{0}^{R} \int_{t>0} \prod_{j \in \psi} \frac{1}{1+\max \left\{e^{t}-1, S_{t h}\right\} r^{\alpha} d_{j}^{-\alpha}}+\frac{1}{3}\left(\prod_{i \in \phi} \frac{1}{1+\left(e^{t}-1\right) r^{\alpha} d_{i}^{-\alpha}}\right. \\
\left.-\prod_{i \in \phi} \frac{1}{1+\max \left\{e^{t}-1, \hat{S}_{t h}\right\} r^{\alpha} d_{i}^{-\alpha}}\right) \mathrm{d} t f_{R}(r) \mathrm{d} r . \\
\text { V. Simulation Results }
\end{gathered}
$$

In this section, we provide the simulation results in order to 456 verify our analytical results In the simulations, we have con- 457 sidered the classic 19 cell system associated with a hexagonal 458 structure having a radius of 1000 meters. A LTE system having 459 a $10 \mathrm{MHz}$ bandwidth, 50 physical resource blocks (PRB) and 460 25 users is considered for each cell. The users are assumed to be 461 uniformly distributed in a cell and similarly, all resource blocks 462 are uniformly shared among users. In other words, if there are 463 $K$ users and $R$ resource blocks then each user is assigned $\frac{R}{K}$ re- 464 source blocks. For each user we generate the channel fading 465 power corresponding to its own channel as well as that corre- 466 sponding to the 18 interferers and then compute the SIR per user 467 per PRB. If a user having an SIR higher than $S_{t h}$ over 25 or more 468 than $25 \mathrm{PRBs}$, then the user is considered to be a cell-centre 469 user, otherwise it is classified as a cell-edge user. For the 470 analytical $\mathrm{CP}_{r}$ computation, (11) and (15) are used for the inde- 471 pendent and correlated cases, respectively. Fig. 4 shows the 472 variation of $\mathrm{CP}_{r}$ as a function of the SINR threshold for FR1, 473 FR3, and the FFR case using both our analytical expressions in 474 (11) and (15) and simulations. Observe in Fig. 4 that the ana- 475 lytical results match the simulation results. It can be seen that 476 for the independent fading case, the $\mathrm{CP}_{r}$ reaches its maximum, 477 when $S_{t h}=T$ and it becomes higher than the FR3 $\mathrm{CP}_{r}$. How- 478 ever, for the fully correlated case, the $\mathrm{CP}_{r}$ becomes maximum, 479 when $S_{t h} \geq T$ and it is equal to the FR3 $\mathrm{CP}_{r}$.

Note that all our results are based on considering Rayleigh 481 fading. However, the results seem to be valid for general fading. 482 For example, Fig. 5 shows the variation of $C P_{r}$ as a function 483 of the SINR threshold by considering Nakagami-m fading 484 using simulations. The $C P_{r}$ is shown for the FR1, FR3 and 485 FFR scenarios for the different values of the Nakagami shape 486 parameter $m$. Similar to the Rayleigh fading scenario, the $C P_{r} 487$ reaches its maximum, when $S_{t h}=T$ and it becomes higher than 488 the FR3 $C P_{r}$. Interestingly, as the Nakagami shape parameter 489 increases, the gap between the optimal FFR $C P_{r}$ and FR3 $C P_{r} 490$ 


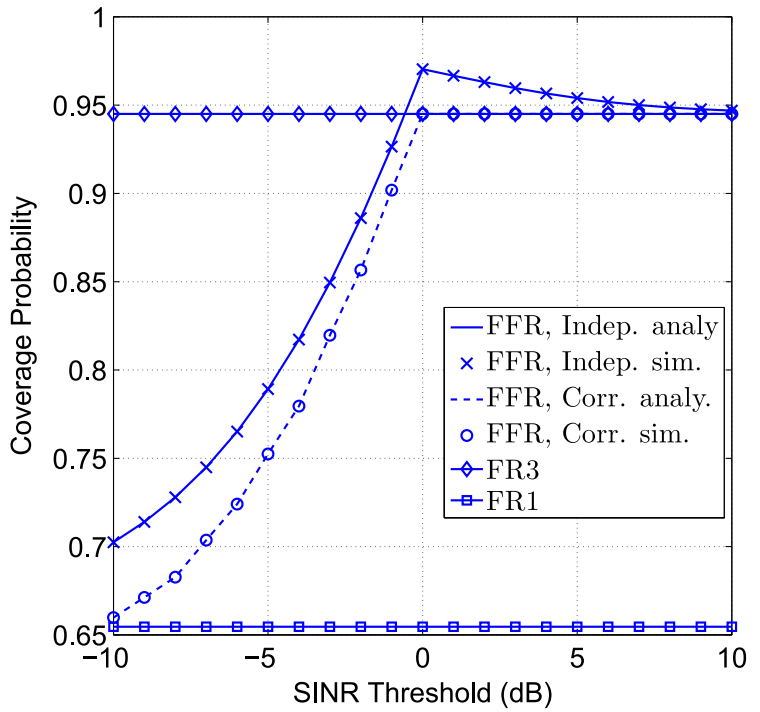

Fig. 4. Coverage probability of FR1, FR3 and FFR evaluated for (11) and (15) with respect to SINR Threshold $S_{t h}$. Here, $T=0 \mathrm{~dB}, \alpha=3.2$ and $N_{t}=N_{r}=1$.

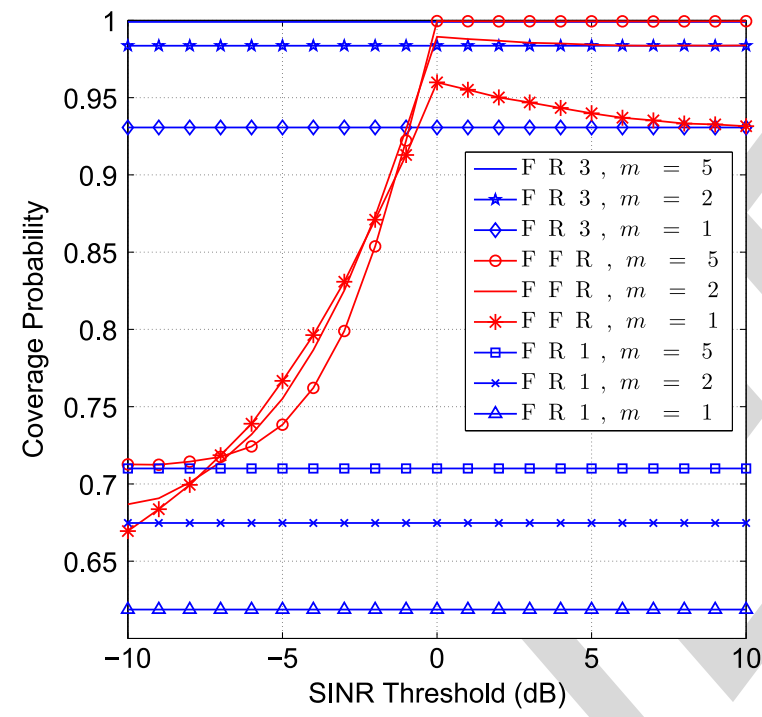

Fig. 5. Coverage probability of FR1, FR3 and FFR for different value of shape parameter for Nakagami-m fading. Here, $T=0 \mathrm{~dB}, \alpha=3$ and $N_{t}=N_{r}=1$.

491 decreases and it almost becomes negligible, when the shape 492 parameter is in excess of $m=5$.

493 Fig. 6 depicts the $\mathrm{CP}_{r}$ of the FFR-aided MU-MIMO and 494 SIMO systems at the optimal value of $S_{t h}$ with respect to the tar495 get SINR. The $\mathrm{CP}_{r}$ of FR1 is also plotted for reference. It can be 496 observed in Fig. 6 that the FR1 $\mathrm{CP}_{r}$ is significantly lower 497 than that of FFR-aided MU-MIMO. The $\mathrm{CP}_{r}$ of the FFR-aided 498 SIMO case is higher than that of the FFR-aided MU-MIMO 499 scenario.

500 Fig. 7 plots the average rate of both the FFR and FR1 systems 501 versus the SINR threshold. For plotting the analytical result, 502 (26) and (34) are used for the independent and correlated case, 503 respectively. Observe that the simulation results closely match 504 the analytical results. Firstly, it can be seen that the FFR 505 achieves the maximum value of the average rate at $3.3 \mathrm{~dB}$, which 506 is the $S_{o p t, R}$ value, as shown in Fig. 3 for a $(1 \times 1)$-antenna sys507 tem. Secondly, it can be observed in Fig. 7 that the average rate

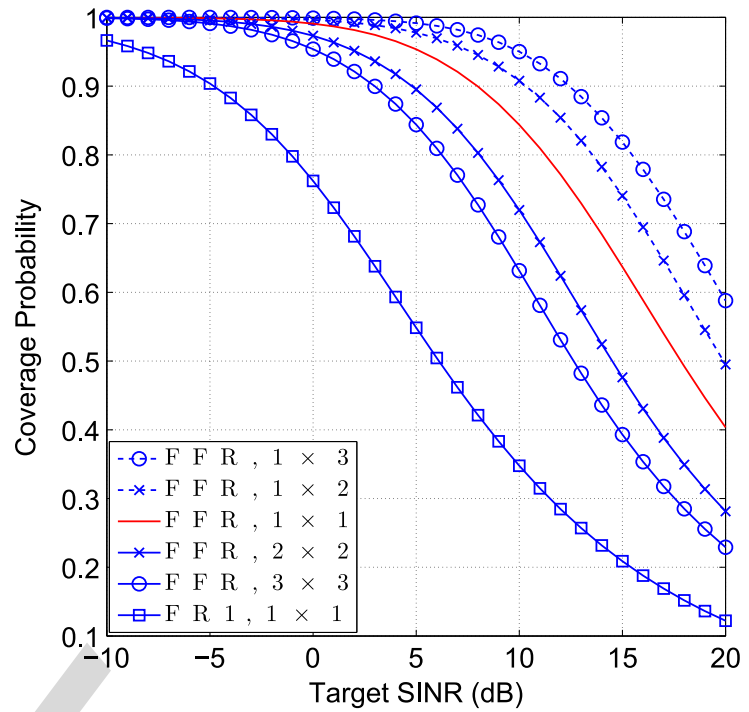

Fig. 6. Coverage probability of both FR1 and of FFR-aided MU-MIMO and SIMO case evaluated for (11) versus the target SINR $T$. Here we have $\alpha=4$ and $S_{t h}=T \mathrm{~dB}, \delta=3$.

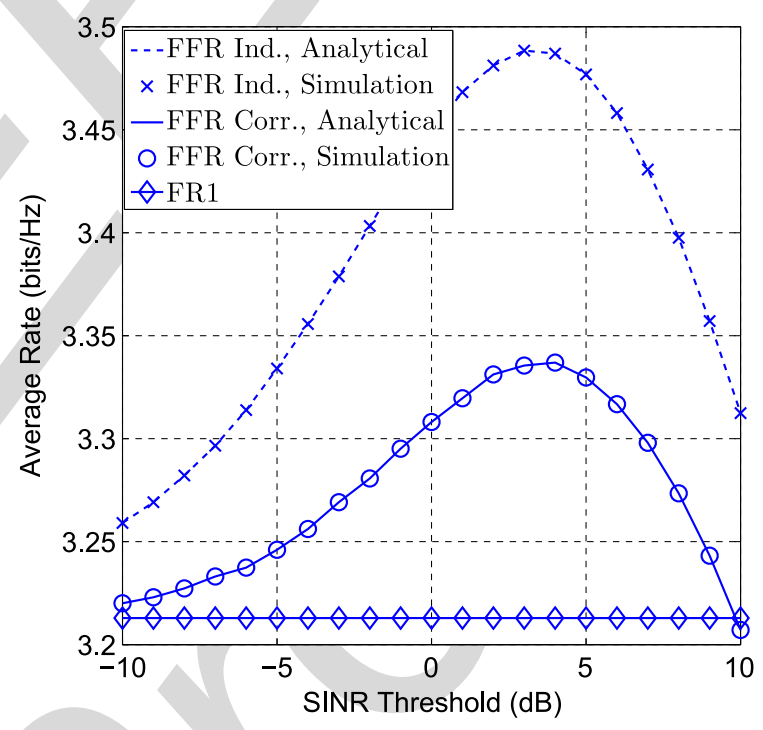

Fig. 7. Average rate of FR1 and FFR versus the SINR threshold. Here we have $\alpha=4, N_{t}=N_{r}=1$. The theoretical results are plotted from Eq. (26) and (34).

is reduced, when the sub-bands are correlated. Furthermore, 508 interestingly, the optimal SINR threshold of the correlated case 509 is nearly the same as the optimal SINR threshold of the inde- 510 pendent fading case. Although, we have considered continuous 511 log-shaped curve mapping between the SINR and the data rate, 512 in practical scenarios, the mapping is given by discrete curves 513 asscociated with different modulation and coding schemes 514 (MCSs). Therefore, we have also provided the average rate 515 versus the SINR threshold based on the specific MCS level 516 using simulation results as shown in Fig. 8. The mapping 517 between SINR and data rate is based on Table 10.1 of the [34]. It 518 can be observed that the value of $S_{\text {opt }, R}$ is the same as observed 519 in Fig. 7. Furthermore, the optimal SINR threshold of the corre- 520 lated case is nearly the same as the optimal SINR threshold of 521 the independent fading scenario. 


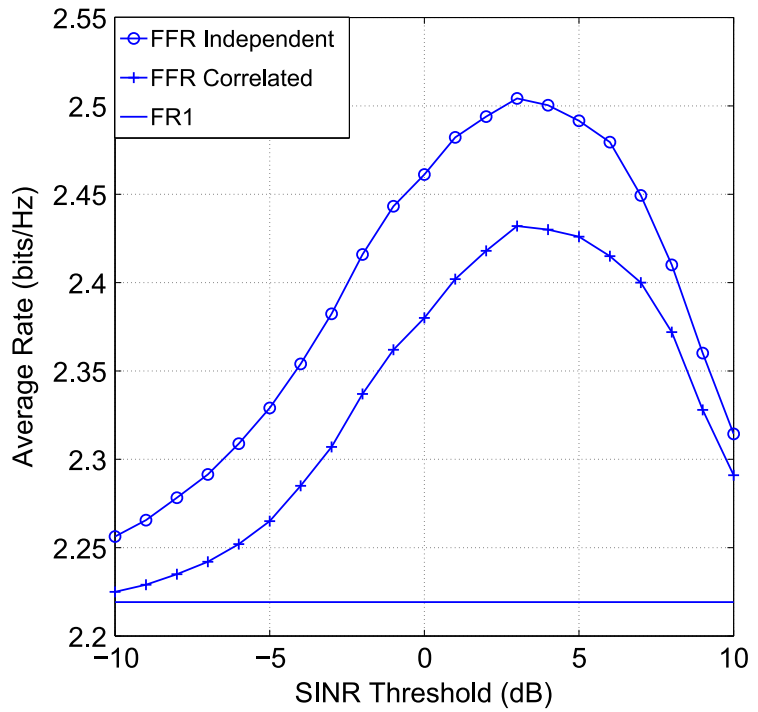

Fig. 8. Average rate of FR1 and FFR using MCS labels versus the SINR threshold. Here we have $\alpha=4, N_{t}=N_{r}=1$.

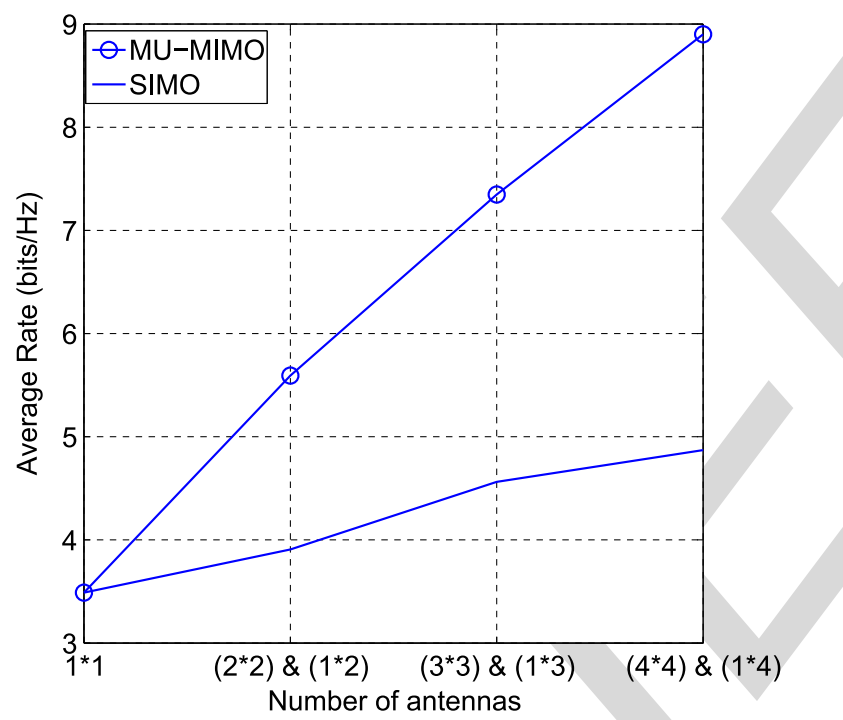

Fig. 9. Maximum average rate achieved by the FFR-aided MU-MIMO and SIMO systems evaluated using (26) and (27) versus the number of antennas for $\alpha=4$.

523 Let us now compare the average rate achieved by the MU524 MIMO and SIMO scenarios at the optimal SINR thresholds. 525 Fig. 9 plots the average rate achieved by the MU-MIMO and 526 SIMO scenarios versus the number of antennas. It is interesting 527 to note that the average rate achieved by the MU-MIMO case 528 is significantly higher than that of the SIMO case. For example, 529 the average rate achieved by the $(2 \times 2)$ MU-MIMO case and 530 by the $(1 \times 3)$ SIMO case are $5.6 \mathrm{bits} / \mathrm{Hz}$ and $4.56 \mathrm{bits} / \mathrm{Hz}$, 531 respectively. In other words, the $(2 \times 2)$ MU-MIMO system 532 achieves a $22.5 \%$ higher rate than the $(1 \times 3)$ SIMO system. 533 However, the overall $\mathrm{CP}_{r}$ achieved by the SIMO case is higher 534 than that of the MU-MIMO case. Now a natural question arises, 535 which of the systems should be chosen by the system designer, 536 since both the $\mathrm{CP}_{r}$ as well as the average rate are important 537 metrics. Based on our results, system designer may opt for the $538(2 \times 2)$ MU-MIMO system over the $(1 \times 3)$ SIMO system, since the gain in average rate is significant and the $\mathrm{CP}_{r}$ degra- 539 dation for $(2 \times 2)$ MU-MIMO is low for lower target SINRs. $\quad 540$

Finally, we have two different expressions for optimal SINR 541 threshold for both the cases, one corresponding to $\mathrm{CP}_{r}\left(S_{t h}=T\right) 542$ and other corresponding to average rate $\left(S_{t h}=T^{\prime}\right)$. To max- 543 imize both $\mathrm{CP}_{r}$ as well as average rate simultaneously, the 544 system designer would have to choose one of these two expres- 545 sions. Now the question arises as to which expression is more 546 appropriate? In order to answer this, we first discuss the benefit 547 of FFR. We see from Figs. 3 and 4 that FFR provides $48 \%$ gain 548 in $\mathrm{CP}_{r}$ and $8.5 \%$ gain in average rate with respect to FR1 at the 549 optimal $S_{t h}$. In other words, FFR provides significantly high 550 gain in $\mathrm{CP}_{r}$ and hence this scheme would be more useful when 551 coverage gain is essentially required. Therefore, FFR-aided 552 MU-MIMO provides both high average rate and satisfactory 553 $\mathrm{CP}_{r}$, since due to MU-MIMO average rate is high and due 554 to FFR scheme $\mathrm{CP}_{r}$ is satisfactory. It can be also noted from 555 Fig. 4 that when $S_{t h}$ is higher than the optimal $S_{t h}$, the loss in 556 $\mathrm{CP}_{r}$ is negligible, while when $S_{t h}$ is lower than the optimal $S_{t h}, 557$ there is significant change in $\mathrm{CP}_{r}$. Hence, for the lower target 558 SINR scenario, i.e., $T<T^{\prime}$, the system designer should choose 559 optimal $S_{t h}$ corresponding to average rate $\left(S_{t h}=T^{\prime}\right)$. On the 560 other hand, for higher target SINR scenario, i.e., $T>T^{\prime}$, the 561 system designer should choose optimal $S_{t h}$ corresponding to $\mathrm{CP}_{r} 562$ $\left(S_{t h}=T\right)$.

\section{CONCLUSION}

564

We have derived expressions for both the $\mathrm{CP}_{r}$ and average 565 rate of MU-MIMO and SIMO systems based on a planned 566 FFR deployment. The impact of frequency-domain correlation 567 between the sub-bands allocated to the FR1 and FR3 regions 568 on the average rate and on the $\mathrm{CP}_{r}$ was analysed in detail, 569 since any practical OFDMA system will typically experience 570 frequency-domain correlation. We analytically determined the 571 optimal SINR threshold, which maximizes the $\mathrm{CP}_{r}$, and also de- 572 termined the optimal SINR threshold (denoted by $S_{\text {opt }, R}$ ), which 573 maximizes the average rate for both the MU-MIMO and SIMO 574 systems considered. It was shown that for the optimal choice 575 of the SINR threshold, the $\mathrm{CP}_{r}$ of the FFR system is higher 576 than that of its FR3 counterpart. The value of $S_{\text {opt }, R}$ increases, 577 when the number of antennas is reduced in a MU-MIMO, where 578 it is assumed that the number of transmit antennas is equal to 579 the number of receive antennas, i.e., $N_{t}=N_{r}=N_{a}$. However, 580 it increases when the number of receive antennas increases in 581 the SIMO scenario. Furthermore, the performance of FFR of 582 the MU-MIMO system and SIMO system are compared. It was 583 shown that $\left(N_{a} \times N_{a}\right)$-element FFR-aided MU-MIMO achieves 584 a significantly higher average rate than $\left(1 \times 2 N_{a}-1\right)$-element 585 SIMO counterpart, but MU-MIMO achieves a lower coverage 586 quality than its SIMO counterpart. However its average rate im- 587 provement is more significant than its $\mathrm{CP}_{r}$ reduction, especially 588 for a lower value of $N_{a}$ and for a lower target SINR. Hence a 589 $(2 \times 2)$ system is preferred over a $(1 \times 3)$ system.

590

A natural extension of this work is to study the FFR-aided 591 MU-MIMO and SIMO system in the context of the cellular 592 uplink [35], [36]. In this study, we have assumed having a 593 fixed transmission power and that the resource blocks are 594 
595 equitably shared by the users. Our future work could consider 596 unequal transmit powers and the unequal allocation of the 597 resource blocks as well as the study of both FFR-aided MU598 MIMO and SIMO systems. Moreover, although strict FFR 599 was considered in the paper, it would also be of substantial 600 interest to study dynamic FFR-aided MU-MIMO and SIMO 601 systems.

602

\section{APPENDIX A}

603 To obtain the $S_{\text {opt }, C}$, we consider the following three possi604 bilities: (i) $S_{t h}<T$, (ii) $S_{t h}=T$, (iii) $S_{t h}>T$.

(i) $S_{t h}<T$ : Let $S_{t h}=T-\Delta$, where $\Delta>0$, then $P_{f}(r)$ can be expressed as in terms of $T$

$$
\begin{aligned}
P_{F}\left(r, S_{t h}<T\right)= & \prod_{i \in \psi}\left(\frac{1}{1+T r^{\alpha} d_{i}^{-\alpha}}\right)^{N_{t}} e^{-T r^{\alpha} \frac{\sigma^{2}}{P}} \\
& +P_{3}(T, r)-P_{3}(T, r) P_{1}(T-\Delta, r) .
\end{aligned}
$$

607 (ii) $S_{t h}=T$ : In this case $P_{f}(r)$ in terms of $T$ can be formu608 lated as

$$
\begin{aligned}
P_{F}\left(r, S_{t h}=T\right)= & \prod_{i \in \psi}\left(\frac{1}{1+T r^{\alpha} d_{i}^{-\alpha}}\right)^{N_{t}} e^{-T r^{\alpha} \frac{\sigma^{2}}{P}} \\
& +P_{3}(T, r)-P_{3}(T, r) P_{1}(T, r) . \\
= & P_{1}(T, r)\left(1-P_{3}(T, r)\right)+P_{3}(T, r) .
\end{aligned}
$$

609 (iii) $S_{t h}>T$ : Let $S_{t h}=T+\Delta$, where $\Delta>0$, then $P_{f}(r)$ in 610 terms of $T$ is given by

$$
\begin{aligned}
P_{F}\left(r, S_{t h}>T\right)= & \prod_{i \in \psi}\left(\frac{1}{1+(T+\Delta) r^{\alpha} d_{i}^{-\alpha}}\right)^{N_{t}} e^{-(T+\Delta) r^{\alpha} \frac{\sigma^{2}}{P}} \\
& +P_{3}(T, r)-P_{3}(T, r) P_{1}(T+\Delta, r) . \\
= & P_{1}(T+\Delta, r)\left(1-P_{3}(T, r)\right)+P_{3}(T, r) .
\end{aligned}
$$

611 Let us now compare the FFR $\mathrm{CP}_{r}$ for $S_{t h}<T$ and $S_{t h}=T$ 612 given by (35) and (36), respectively. Since we have $P_{1}(T-\Delta$, $613 r)>P_{1}(T, r)$, this implies that $P_{F}\left(r, S_{t h}<T\right)<P_{F}\left(r, S_{t h}=T\right)$. 614 Similarly, we compare the FFR-aided $\mathrm{CP}_{r}$ for $S_{t h}=T$ and $615 S_{t h}>T$ given by (37) and (38), respectively. Since $P_{1}(T+\Delta$, $616 r)<P_{1}(T, r)$, this implies that $P_{F}\left(r, S_{t h}=T\right)>P_{F}\left(r, S_{t h}>T\right)$. 617 Thus, FFR achieves the maximum achievable $\mathrm{CP}_{r}$ when $S_{t h}=T$. 618 Note that when one chooses the SINR threshold to be $S_{o p t, C}$, 619 then the $\mathrm{CP}_{r}$ of FFR is higher than that of FR3 since we 620 have $C P_{F}\left(r, S_{t h}=T\right)=P_{1}(T, r)\left(1-P_{3}(T, r)\right)+P_{3}(T, r)>$ $621 P_{3}(T, r)$. The reason for this behaviour is as follows: only users 622 having a low SINR (low fading gain for the desired signal 623 and/or high fading gain for the interfering signal) move to the 624 cell-edge region and they experience a new independent fading 625 gain at the cell-edge region. In other words, the increase in FFR $626 \mathrm{CP}_{r}$ over the $\mathrm{FR} 3 \mathrm{CP}_{r}$ is due to the sub-band diversity gains 627 which is achieved by the system, when the users move from the 628 cell-centre to the cell-edge.

\section{APPENDIX B}

Since a cell-centre user is associated with $\eta_{t}(r)>S_{t h}$, the 630 average rate $R_{c}(r)$ of the cell-centre users of the FFR system can 631 be written as $R_{c}(r)=E\left[\ln \left(1+\eta_{t}(r)\right) \mid \eta_{t}(r)>S_{t h}\right]$ Similarly, 632 since a cell-edge user has $\eta_{t}(r)<S_{t h}$, the average rate $R_{e}(r)$ of 633 the cell-edge users in the FFR system can be written as $R_{e}(r)=634$ $E\left[\ln \left(1+\hat{\eta}_{t}(r)\right) \mid \eta_{t}(r)<S_{t h}\right]$. Now, the average rate $R_{f}(r)$ of the 635 FFR system can be written as

$$
R_{f}(r)=R_{c}(r) P\left[\eta_{t}(r)>S_{t h}\right]+\frac{1}{3} R_{e}(r) P\left[\eta_{t}(r)<S_{t h}\right] .
$$

Here the first term denotes the average rate contributed by the 637 cell-centre users, while the second term denotes the contribu- 638 tion of the cell-edge users. Recall that the frequency reuse $\frac{1}{3}$ is 639 invoked for the cell-edge users. In other words, only one third 640 of the cell-edge frequency $\left(F_{1}+F_{2}+F_{3}\right)$ is used for the cell- 641 edge users and hence the factor $\frac{1}{3}$ is multiplied in the above ex- 642 pression. Using the methods outlined in Section IV-A, 643 $R_{c}(r) P\left[\eta(r)>S_{t h}\right]$ can be written as 644

$$
\begin{aligned}
R_{c}(r) P\left[\eta_{t}(r)>S_{t h}\right] & \left.\left.=\int_{t>0} P\left[\ln \left(1+\eta_{t}(r)\right)>t, \eta_{t}\right) r\right)>S_{t h}\right] \mathrm{dt} \\
& =\int_{t>0} P\left[\eta_{t}(r)>\max \left\{e^{t}-1, S_{t h}\right\}\right] \mathrm{dt} . \quad
\end{aligned}
$$

Using (3) and (4), this can be further simplified to

$R_{c}(r) P\left[\eta_{t}(r)>S_{t h}\right]=\int \prod_{t>0}\left(\frac{1}{1+\max \left\{e^{t}-1, S_{t h}\right\} r^{\alpha} d_{j}^{-\alpha}}\right)^{N_{t}} \mathrm{~d} t$.

Again, similar to Section IV-A, we can write $R_{e}(r)$ as

$$
\begin{aligned}
R_{e}(r) & =\int_{t>0} \frac{P\left[\ln \left(1+\hat{\eta}_{t}(r)\right)>t, \eta_{t}(r)<S_{t h}\right]}{P\left[\eta_{t}(r)<S_{t h}\right]} \mathrm{dt} \\
& =\int_{t>0} \frac{P\left[\hat{\eta}_{t}(r)>\left(e^{t}-1\right), \eta_{t}(r)<S_{t h}\right]}{P\left[\eta_{t}(r)<S_{t h}\right]} \mathrm{dt} .
\end{aligned}
$$

Since $g$ and $\hat{g}$ are i.i.d as well as $h_{i}$ and $\hat{h}_{i}$ are also i.i.d, hence 647 $R_{e}(r)$ can be written as

$$
R_{e}(r)=\int \prod_{t>0}\left(\frac{1}{i \in \phi}\right)^{N_{t}} \mathrm{~d} t
$$

Finally substituting back (41) and (43) into (39) and after aver- 649 aging over the spatial dimension, the average rate of the FFR 650 system is given by

$$
\begin{aligned}
R_{f}=\int_{0}^{R} \int_{t>0}( & \prod_{j \in \psi}\left(\frac{1}{1+\max \left\{e^{t}-1, S_{t h}\right\} r^{\alpha} d_{j}^{-\alpha}}\right)^{N_{t}} \\
& \left.+\frac{1}{3} \prod_{i \in \phi} \frac{P\left[\eta_{t}(r)<S_{t h}\right]}{\left(1+\left(e^{t}-1\right) r^{\alpha} d_{i}^{-\alpha}\right)^{N_{t}}}\right) \mathrm{d} t f_{R}(r) \mathrm{d} r
\end{aligned}
$$




$$
\begin{aligned}
R_{f}=\int_{0}^{R} \int_{t>0}\left(\prod_{j \in \psi}\left(\frac{1}{1+\max \left\{e^{t}-1, S_{t h}\right\} r^{\alpha} d_{j}^{-\alpha}}\right)^{N_{t}}\right. \\
\left.+\frac{1}{3} \prod_{i \in \phi} \frac{P\left[\eta_{t}(r)<S_{t h}\right]}{\left(1+\left(e^{t}-1\right) r^{\alpha} d_{i}^{-\alpha}\right)^{N_{t}}}\right) \mathrm{d} t f_{R}(r) \mathrm{d} r .
\end{aligned}
$$

654 To maximize the rate $R_{f}$, we have to differentiate $R_{f}$ with re655 spect to $S_{t h}$. In order to do that we split the first part of the integ656 rand of $R_{f}$ as given in (46), shown at the bottom of the page. 657 Upon substituting $P\left[\eta_{t}(r)<S_{t h}\right]=1-\prod_{j \in \psi}\left(\frac{1}{1+S_{t h} r^{\alpha} d_{j}^{-\alpha}}\right)^{N_{t}}$ 658 into Eq. (45), $R_{f}$ can be rewritten as given in (47), shown at the bottom of the page. Using Leibniz's rule, ${ }^{6}$ while differentiating 659 $R_{f}$ with respect to $S_{t h}$, we obtain (48), shown at the bottom of 660 the page. Simplifying $\frac{d R_{f}}{d S_{t h}}$ and equating it to zero, one obtains 661 $\frac{d R_{f}}{d S_{t h}}$ as given in (48). The solution of the integral given in (48) 662 gives the optimal $S_{t h}$, namely $S_{o p t, R}$, but obtaining $S_{o p t, R}$ in 663 a closed form is a challenging problem, as the distances $d_{i} \mathrm{~s} 664$ are also a function of $r$. Hence, we find the value of $S_{o p t, R}$ by 665 solving (48) numerically (using Mathematica (or Matlab)). 666 Note that the optimal value of $S_{t h}$ is calculated at the time of 667 network planning with the aid of Mathematica (or Matlab) 668 to obtain the numerical values off line. We have investigated 669 $S_{o p t, R}$ as a function of the path loss exponent, of the number of 670 transmit antennas, etc.

${ }^{6}$ Leibniz's rule states that if $f(x, \theta)$ is a function such that $\frac{d}{d \theta} f(x, \theta)$ exist, and it is continuous, then we have $\frac{d}{d \theta}\left(\int_{a(\theta)}^{b(\theta)} f(x, \theta) d x\right)=\int_{a(\theta)}^{b(\theta)} \frac{d}{d \theta}(f(x, \theta)) d x+$ $f(b(\theta), \theta) \frac{d}{d \theta} b(\theta)-f(a(\theta), \theta) \frac{d}{d \theta} a(\theta)$.

$$
\int_{t>0} \prod_{j \in \psi}\left(\frac{1}{1+\max \left\{e^{t}-1, S_{t h}\right\} r^{\alpha} d_{j}^{-\alpha}}\right)^{N_{t}} \mathrm{~d} t=\int_{t>0}^{\ln \left(1+S_{t h}\right)} \prod_{j \in \psi}\left(\frac{1}{1+S_{t h} r^{\alpha} d_{j}^{-\alpha}}\right)^{N_{t}} \mathrm{~d} t+\int_{\ln \left(1+S_{t h}\right)}^{\infty} \prod_{j \in \psi}\left(\frac{1}{1+\left(e^{t}-1\right) r^{\alpha} d_{j}^{-\alpha}}\right)^{N_{t}} \mathrm{~d} t
$$

$$
\begin{aligned}
& R_{f}=\int_{0}^{R}\left(\prod_{j \in \psi} \frac{\ln \left(1+S_{t h}\right)}{\left(1+S_{t h} r^{\alpha} d_{j}^{-\alpha}\right)^{N_{t}}}+\int_{\ln \left(1+S_{t h}\right)}^{\infty} \prod_{j \in \psi}\left(\frac{1}{1+\left(e^{t}-1\right) r^{\alpha} d_{j}^{-\alpha}}\right)^{N_{t}} \mathrm{~d} t\right. \\
& +\left(1-\prod_{j \in \psi}\left(\frac{1}{1+S_{t h} r^{\alpha} d_{j}^{-\alpha}}\right)^{N_{t}}\right) \underbrace{\frac{1}{3} \int_{t>0} \prod_{i \in \phi}\left(\frac{1}{1+\left(e^{t}-1\right) r^{\alpha} d_{i}^{-\alpha}}\right)^{N_{t}} \mathrm{~d} t}_{K(r)}) f_{R}(r) \mathrm{d} r .
\end{aligned}
$$

$$
\begin{aligned}
& \frac{d R_{f}}{d S_{t h}}=\int_{0}^{R}\left(\frac{\frac{\prod_{j \in \psi}\left(1+S_{t h} r^{\alpha} d_{j}^{-\alpha}\right)^{N_{t}}}{1+S_{t h}}-\ln \left(1+S_{t h}\right) \frac{d}{d S_{t h}}\left(\prod_{j \in \psi}\left(1+S_{t h} r^{\alpha} d_{j}^{-\alpha}\right)^{N_{t}}\right)}{\left(\prod_{j \in \psi}\left(1+S_{t h} r^{\alpha} d_{j}^{-\alpha}\right)\right)^{2 N_{t}}}\right. \\
& -\prod_{j \in \psi} \frac{1}{\left(1+S_{t h} r^{\alpha} d_{j}^{-\alpha}\right)^{N_{t}}}\left(\frac{1}{1+S_{t h}}\right)+\frac{K(r) \frac{d}{d S_{t h}}\left(\prod_{j \in \psi}\left(1+S_{t h} r^{\alpha} d_{j}^{-\alpha}\right)^{N_{t}}\right)}{\left(\prod_{j \in \psi}\left(1+S_{t h} r^{\alpha} d_{j}^{-\alpha}\right)\right)^{2 N_{t}}} f_{R}(r) \mathrm{d} r . \\
& \frac{d R_{f}}{d S_{t h}}=\int_{0}^{R}\left(\frac{\left(K(r)-\ln \left(1+S_{t h}\right)\right) \sum_{i \in \psi}\left(1+S_{t h} r^{\alpha} d_{i}^{-\alpha}\right)^{N_{t}-1} r^{\alpha} d_{i}^{-\alpha}\left(\prod_{j \in \psi \backslash i}\left(1+S_{t h} r^{\alpha} d_{j}^{-\alpha}\right)^{N_{t}}\right)}{\left(\prod_{j \in \psi}\left(1+S_{t h} r^{\alpha} d_{j}^{-\alpha}\right)\right)^{2 N_{t}}}\right) f_{R}(r) \mathrm{d} r=0
\end{aligned}
$$




\section{REFERENCES}

[1] G. Boudreau et al., "Interference coordination and cancellation for $4 \mathrm{G}$ networks," IEEE Commun. Mag., vol. 47, no. 4, pp. 74-81, Apr. 2009.

[2] N. Himayat, S. Talwar, A. Rao, and R. Soni, "Interference management for 4G cellular standards [WIMAX/LTE UPDATE]," IEEE Commun. Mag., vol. 48, no. 8, pp. 86-92, Aug. 2010.

[3] F. Wang et al., "Mobile WiMAX systems: Performance and evolution," IEEE Commun. Mag., vol. 46, no. 10, pp. 41-49, Oct. 2008.

[4] D. Astely et al., "LTE: The evolution of mobile broadband," IEEE Commun. Mag., vol. 47, no. 4, pp. 44-51, Apr. 2009.

5] A. S. Hamza, S. S. Khalifa, H. S. Hamza, and K. Elsayed, "A survey on inter-cell interference coordination techniques in OFDMA-based cellular networks," IEEE Commun. Surveys Tuts., vol. 15, no. 4, pp. 1642-1670, 4th Quart. 2013.

[6] R. Y. Chang, Z. Tao, J. Zhang, and C.-C. J. Kuo, "A graph approach to dynamic Fractional Frequency Reuse (FFR) in multi-cell OFDMA networks," in Proc. IEEE ICC, Jun. 2009, pp. 1-6.

[7] M. Assaad, "Optimal Fractional Frequency Reuse (FFR) in multicellular OFDMA system," in Proc. IEEE 68th VTC-Fall, Sep. 2008, pp. 1-5.

[8] Z. Xu, G. Ye Li, C. Yang, and X. Zhu, "Throughput and optimal threshold for FFR schemes in OFDMA cellular networks," IEEE Trans. Wireless Commun., vol. 11, no. 8, pp. 2776-2785, Aug. 2012.

[9] T. Novlan, J. G. Andrews, I. Sohn, R. K. Ganti, and A. Ghosh, "Comparison of fractional frequency reuse approaches in the OFDMA cellular downlink," in Proc. IEEE Global Telecommun. Conf., 2010, pp. $1-5$.

0] T. D. Novlan, R. K. Ganti, A. Ghosh, and J. G. Andrews, "Analytical evaluation of fractional frequency reuse for OFDMA cellular networks," IEEE Trans. Wireless Commun., vol. 10, no. 12, pp. 4294-4305, Dec. 2011.

1] H. Zhuang and T. Ohtsuki, "A model based on Poisson point process for analyzing MIMO heterogeneous networks utilizing fractional frequency reuse," IEEE Trans. Wireless Commun., vol. 13, no. 12, pp. 6839-6850, Dec. 2014.

2] A. Mahmud and K. A. Hamdi, "A unified framework for the analysis of fractional frequency reuse techniques," IEEE Trans. Commun., vol. 62, no. 10, pp. 3692-3705, Oct. 2014.

3] D. G. Gonzalez, M. Garcia-Lozano, S. Ruiz Boque, and D. S. Lee, "Optimization of soft frequency reuse for irregular LTE macrocellular networks," IEEE Trans. Wireless Commun., vol. 12, no. 5, pp. 2410-2423, May 2013.

4] X. Tao, F. Xu, W. ur Rehman, Y. Xu, and X. Li, "A generic mathematical model based on fuzzy set theory for frequency reuse in cellular networks," IEEE J. Sel. Areas Commun., vol. 31, no. 5, pp. 861-869, May 2013.

15] L.-C. Wang and C.-J. Yeh, "3-cell network MIMO architectures with sectorization and fractional frequency reuse," IEEE J. Sel. Areas Commun., vol. 29, no. 6, pp. 1185-1199, Jun. 2011.

16] S. Kumar and S. Kalyani, "Impact of correlated interferers on coverage and rate of FFR and SFR schemes," IEEE Trans. Veh. Technol., to be published.

17] H. Chang and I. Rubin, "Optimal downlink and uplink fractional frequency reuse in cellular wireless networks" IEEE Trans. Veh. Technol., to be published.

[18] O. Aliu, M. Mehta, M. Imran, A. Karandikar, and B. Evans, "A new cellular-automata-based fractional frequency reuse scheme," IEEE Trans. Veh. Technol., vol. 64, no. 4, pp. 1535-1547, Apr. 2015.

9] Q. Li, R. Hu, Y. Xu, and Y. Qian, "Optimal fractional frequency reuse and power control in the heterogeneous wireless networks," IEEE Trans. Wireless Commun., vol. 12, no. 6, pp. 2658-2668, Jun. 2013.

20] J. Zhang, R. Zhang, G. Li, and L. Hanzo, "Distributed antenna systems in fractional-frequency-reuse-aided cellular networks," IEEE Trans. Veh. Technol., vol. 62, no. 3, pp. 1340-1349, Mar. 2013.

[21] S. Kumar, S. Kalyani, and K. Giridhar, "Spectrum allocation for ICIC based picocell," IEEE Trans. Veh. Technol., to be published.

[22] F. Jin, R. Zhang, and L. Hanzo, "Fractional frequency reuse aided twin-layer femtocell networks: Analysis, design and optimization," IEEE Trans. Commun., vol. 61, no. 5, pp. 2074-2085, May 2013.

23] W. S. Jeon, J. Kim, and D. G. Jeong, "Downlink radio resource partitioning with fractional frequency reuse in femtocell networks," IEEE Trans. Veh. Technol., vol. 63, no. 1, pp. 308-321, Jan. 2014.

24] F. Wang and W. Wang, "Analytical evaluation of femtocell deployment in cellular networks using fractional frequency reuse," IET Commun., vol. 8, no. 9, pp. 1599-1608, Jun. 2014.

25] N. Saquib, E. Hossain, and D. I. Kim, "Fractional frequency reuse for interference management in lte-advanced hetnets," IEEE Wireless Commun., vol. 20, no. 2, pp. 113-122, Apr. 2013.
[26] J. Y. Lee, S. J. Bae, Y. M. Kwon, and M. Y. Chung, "Interference anal- 748 ysis for femtocell deployment in OFDMA systems based on fractional 749 frequency reuse," IEEE Commun. Lett., vol. 15, no. 4, pp. 425-427, 750 Apr. 2011.

[27] Q. H. Spencer, A. L. Swindlehurst, and M. Haardt, "Zero-forcing methods 752 for downlink spatial multiplexing in multiuser MIMO channels," IEEE 753 Trans. Signal Process., vol. 52, no. 2, pp. 461-471, Feb. 2004.

[28] D. Gesbert, M. Kountouris, R. W. Heath, C.-B. Chae, and T. Salzer, 755 "Shifting the MIMO paradigm," IEEE Signal Process. Mag., vol. 24, 756 no. 5, pp. 36-46, Sep. 2007.

[29] S. Jafar and A. Goldsmith, "Isotropic fading vector broadcast channels: 758 The scalar upper bound and loss in degrees of freedom," IEEE Trans. Inf. 759 Theory, vol. 51, no. 3, pp. 848-857, Mar. 2005.

[30] S. Catreux, P. Driessen, and L. Greenstein, "Simulation results for an 761 interference-limited multiple-input multiple-output cellular system," 762 IEEE Commun. Lett., vol. 4, no. 11, pp. 334-336, Nov. 2000.

[31] J. G. Andrews, W. Choi, and R. W. Heath, "Overcoming interference in 764 spatial multiplexing MIMO cellular networks," IEEE Wireless Commun., 765 vol. 14, no. 6, pp. 95-104, Dec. 2007.

[32] D. Tse and P. Viswanath, Fundamentals of Wireless Communication. 767 Cambridge, U.K.: Cambridge Univ. Press, 2005.

[33] K. B. Baltzis, "Hexagonal vs circular cell shape: A comparative analysis 769 and evaluation of the two popular modeling approximations," in Cellular 770 Networks-Positioning, Performance Analysis, Reliability, A. Melikov Ed. 771 Rijeka, Croatia: Intechopen, 2011.

[34] S. Sesia, I. Toufik, and M. Baker, LTE-The UMTS Long Term Evolution: 773 From Theory to Practice. Hoboken, NJ, USA: Wiley, 2011. [Online]. 774 Available: https://books.google.co.uk/books?id=beIaPXLzYKcC 775

[35] H. Tabassum, F. Yilmaz, Z. Dawy, and M.-S. Alouini, "A framework 776 for uplink intercell interference modeling with channel-based schedul- 777 ing," IEEE Trans. Wireless Commun., vol. 12, no. 1, pp. 206-217, 778 Jan. 2013.

[36] H. Tabassum, Z. Dawy, M. S. Alouini, and F. Yilmaz, "A generic 780 interference model for uplink OFDMA networks with fractional fre- 781 quency reuse," IEEE Trans. Veh. Technol., vol. 63, no. 3, pp. 1491-1497, 782 Mar. 2014.

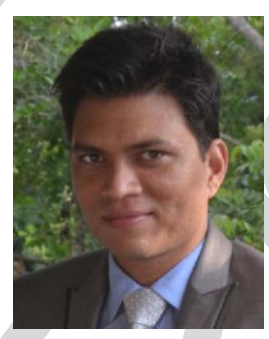

Suman Kumar received the B.Tech. degree in elec- 784 tronics and communication engineering from the 785 Future Institute of Engineering and Management, 786 Kolkata, India, in 2010. He is currently pursuing 787 the Ph.D. degree in the Department of Electrical 788 Engineering, Indian Institute of Technology, Madras, 789 India. He is the recipient of a Best Paper Award at 790 ICWMC-2012 held at Venice, Italy.

His research interests are broadly in the areas of 792 performance analysis of mobile broadband wireless 793 networks including frequency reuse, HetNets, hyper- 794 geometric functions, and generalized fading models.

795

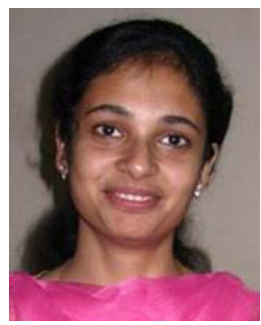

Sheetal Kalyani received the B.E. degree in elec- 796 tronics and communication engineering from the 797 Sardar Patel University, Gujarat, India, in 2002 and 798 the Ph.D. degree in electrical engineering from the 799 Indian Institute of Technology, Madras, India, in 800 2008. She was a Senior Research Engineer in Cen- 801 tre of Excellence in Wireless Technology, Chennai, 802 India, from 2008 to 2012. She is currently an As- 803 sistant Professor in the Department of Electrical 804 Engineering, Indian Institute of Technology, Madras. 805

Her current research interests include HetNets, 806 extreme value theory, hypergeometric functions, generalized fading models, 807 and statistical learning algorithms for prediction. 


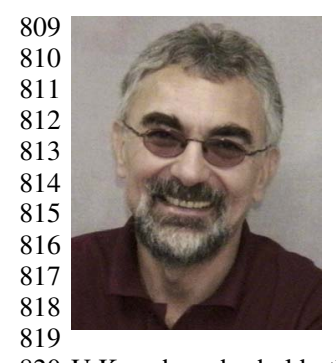

Lajos Hanzo (M'91-SM'92-F'04) received the degree in electronics in 1976 and his doctorate in 1983 from the Technical University of Budapest, Budapest, Hungary. In 2009, he was awarded an honorary doctorate by the Technical University of Budapest, and in 2015 by the University of Edinburgh. During his 38-year career in telecommunications, he has held various research and academic posts in Hungary, Germany, and the U.K. Since 1986, he has been with the School of Electronics and Computer Science, University of Southampton, 820 U.K., where he holds the Chair in telecommunications. He has successfully 821 supervised about 100 Ph.D. students, co-authored 20 John Wiley/IEEE Press 822 books on mobile radio communications totalling in excess of 10000 pages, 823 published over 1500 research entries at IEEE Xplore, acted both as TPC 824 and General Chair of IEEE conferences, presented keynote lectures, and 825 been awarded a number of distinctions. Currently, he is directing a 60826 strong academic research team, working on a range of research projects 827 in the field of wireless multimedia communications sponsored by industry, 828 the Engineering and Physical Sciences Research Council (EPSRC) U.K., 829 the European Research Council's Advanced Fellow Grant, and the Royal 830 Society's Wolfson Research Merit Award. He is an enthusiastic supporter of 831 industrial and academic liaison and he offers a range of industrial courses. $832 \mathrm{He}$ is also a Governor of the IEEE Vehicular Technology Society. During 833 2008-2012, he was the Editor-in-Chief of the IEEE Press and a Chaired 834 Professor also at Tsinghua University, Beijing. His research is funded by 835 the European Research Council's Senior Research Fellow Grant. Dr. Hanzo 836 has over 22000 citations. For further information on research in progress 837 and associated publications, please refer to http://www-mobile.ecs.soton.ac.uk.

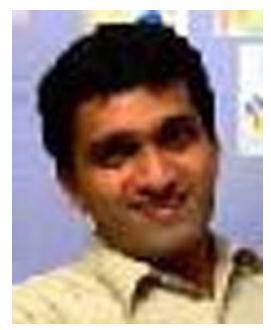

K. Giridhar (M'XX) received the B.Sc. degree in $838 \quad \mathbf{A Q 7}$ applied sciences from PSG College of Technology, 839 Coimbatore, India, the M.E. degree in electrical 840 communications from Indian Institute of Science, 841 Bangalore, India, and the Ph.D. degree in electri- 842 cal engineering from University of California, Santa 843 Barbara, Santa Barbara, CA, USA.

844

$\mathrm{He}$ is a Professor at the Indian Institute of Tech- 845 nology Madras (www.iitm.ac.in), Chennai. During 846 1989 and 1990, he was a Member of Research Staff 847 at CRL, Bharat Electronics, Bangalore, and during 848 1993 and 1994, was a Research Affiliate in electrical engineering at Stanford 849 University, Stanford, CA, USA. Since 1994, he has been with the Department 850 of Electrical Engineering, Indian Institute of Technology, Madras (ITTM). He 851 has been a Visiting Faculty at Sri Sathya Sai Institute of Higher Learning, 852 Prasanthi Nilayam, Andhra Pradesh, and at Stanford University. His research 853 interests are broadly in the areas of adaptive signal processing and wireless 854 communications systems, with an emphasis on various transceiver algorithms, 855 custom air-interface design for strategic applications, and performance analysis 856 of mobile broadband wireless networks including HetNets.

Dr. Giridhar is a member of the Telecommunications and Computer 858 Networks (TeNeT) Group (www.tenet.res.in) at IITM. He actively collaborates 859 with the Center of Excellence in Wireless Technology (www.cewit.org.in) on 860 MIMO-OFDM broadband access research, resulting in several contributions to 861 IEEE $802.16 \mathrm{~m}$, and currently on proposals to LTE-A and 5G forums. He serves 862 as a consultant to many telecom \& VLSI companies in India, and was on a 863 sabbatical in 2004-2005 with Beceem Communications. 


\section{AUTHOR QUERIES}

\section{AUTHOR PLEASE ANSWER ALL QUERIES}

AQ1 = Please provide keywords.

AQ2 = Please provide department name for IIT, Madras.

AQ3 = Please provide publication update in Ref. [16].

AQ4 = Please provide publication update in Ref. [17].

AQ5 = Please provide publication update in Ref. [21].

AQ6 = Please provide page range of chapter for Ref. [33].

AQ7 = Please provide membership history for K. Giridhar.

\section{END OF ALL QUERIES}




\section{- Coverage Probability and Achievable Rate Analysis of FFR-Aided Multi-User OFDM-Based MIMO and SIMO Systems}

5 Abstract-Expressions are derived for the coverage probability 6 and average rate of both multi-user multiple input multiple output 7 (MU-MIMO) and single input multiple output (SIMO) systems 8 in the context of a fractional frequency reuse (FFR) scheme. In 9 particular, given a reuse region of $\frac{1}{3}(\mathrm{FR3})$ and a reuse region of 101 (FR1) as well as a signal-to-interference-plus-noise-ratio (SINR) 11 threshold $S_{t h}$, which decides the user assignment to either the FR1 12 or FR3 regions, we theoretically show that: 1) the optimal choice 13 of $S_{t h}$ which maximizes the coverage probability is $S_{t h}=T$, where $14 \boldsymbol{T}$ is the target SINR required for ensuring adequate coverage, and 152 ) the optimal choice of $S_{t h}$ which maximizes the average rate is 16 given by $S_{t h}=T^{\prime}$, where $T^{\prime}$ is a function of the path loss exponent, 17 the number of antennas and of the fading parameters. The impact 18 of frequency domain correlation amongst the OFDM sub-bands 19 allocated to the FR1 and FR3 cell-regions is analysed and it is 20 shown that the presence of correlation reduces both the coverage 21 probability and the average throughput of the FFR network. 22 Furthermore, the performance of our FFR-aided MU-MIMO and 23 SIMO systems is compared. Our analysis shows that the $(2 \times 2)$ 24 MU-MIMO system achieves $22.5 \%$ higher rate than the $(1 \times 3)$ 25 SIMO system and for lower target SINRs, the coverage probability 26 of a $(2 \times 2)$ MU-MIMO system is comparable to a $(1 \times 3)$ SIMO 27 system. Hence the former one may be preferred over the latter. 28 Our simulation results closely match the analytical results.

29 Index Terms-Author, please supply index terms/keywords for 30 your paper. To download the IEEE Taxonomy go to http://www. 31 ieee.org/documents/taxonomy_v101.pdf.

\section{INTRODUCTION}

33

34 RTHOGONAL frequency division multiple access (OFDMA) based systems maintain orthogonality among 35 the intra-cell users, but the radical OFDMA system deploy36 ments relying on a frequency reuse factor of unity suffer from 37 inter-cell interference. As a remedy, inter-cell interference coor38 dination (ICIC) schemes have been designed for minimizing the 39 co-channel interference [1]. Fractional frequency reuse (FFR) 40 [2] constitutes a low complexity ICIC scheme, which has been 41 proposed for OFDMA based wireless networks such as IEEE 42 WiMAX [3] and 3GPP LTE [4].

Manuscript received January 18, 2015; revised June 5, 2015; accepted August 1,2015. The associate editor coordinating the review of this paper and approving it for publication was O. Oyman.

S. Kumar, S. Kalyani, and K. Giridhar are with the Indian Institute of Technology Madras, Chennai 600 036, India (e-mail: ee10d040@ee.iitm.ac.in; skalyani@ee.iitm.ac.in; giri@ee.iitm.ac.in).

L. Hanzo is with the School of Electrical and Computer Science, University of Southampton, Southampton SO17 1BJ, U.K. (e-mail: lh@ecs.soton.ac.uk).

Color versions of one or more of the figures in this paper are available online at http://ieeexplore.ieee.org.

Digital Object Identifier 10.1109/TCOMM.2015.2465907

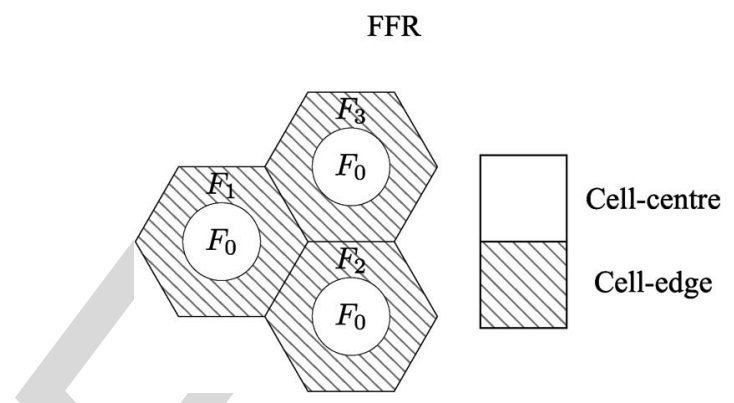

Fig. 1. Frequency allocation in FFR for three neighbouring cells with $\delta=3$. The cell-centre users of all the cells rely on a common frequency band $F_{0}$, while the cell-edge users of the three cells occupy different frequency bands, namely $F_{1}, F_{2}$ and $F_{3}$.

Explicitly, FFR is a combination of frequency reuse 1 (FR1) 43 and frequency reuse $\frac{1}{\delta}(\mathrm{FR} \delta)$. FR1 allocates all the frequencies 44 to each cell, leading to a unity spatial reuse, hence results in 45 a low-quality coverage due to the excessive inter-cell interfer- 46 ence. On the other hand, FR $\delta$ allocates a fraction of $\frac{1}{\delta}$ of the 47 frequencies to each cell and therefore reduces the area-spectral- 48 efficiency, but improves the SINR. FFR strikes an attractive 49 trade-off by exploiting the advantages of both FR 1 and FR $\delta$ by 50 relying on FR1 for the cell-centre users i.e. for those users who 51 would experience less interference from the other cells, because 52 they are close to their serving base station (BS). By contrast, 53 $\mathrm{FR} \delta$ is invoked for the cell-edge users i.e. for those users who 54 would experience high interference afflicted by the co-channel 55 signals emanating from the neighbouring cells in case of FR1, 56 because they are far from their serving BS. Typically, there 57 are two basic modes of FFR deployment: static and dynamic 58 FFR [1]. In this paper, we consider the more practical static 59 FFR scheme, where all the parameters are configured and kept 60 fixed over a certain period of time [5]. Fig. 1 depicts a typical 61 frequency allocation in the context of the FFR scheme for three 62 adjacent cells, where $F_{1}, F_{2}$ and $F_{3}$ each use $x \%$ of the total 63 spectrum, hence $F_{0}$ uses $(100-3 x) \%$ of the spectrum. 64

FFR schemes have been lavishly studied using both system 65 level simulations and theoretical analysis [6]-[11]. The optimiz- 66 ation of FFR relying on a distance threshold ${ }^{1}$ or SINR threshold ${ }^{2} 67$

\footnotetext{
${ }^{1}$ Based on a pre-determined distance from the BS, the subscribers are divided into cell-centre as well as cell-edge users and hence here the design parameter is a distance threshold $\left(R_{t h}\right)$.

${ }^{2}$ Based on a pre-determined SINR, the subscribers are divided into cellcentre as well as cell-edge users and here the design parameter is the SINR threshold $\left(S_{t h}\right)$.
} 
68 has been studied using graph theory in [6] and convex optimiza69 tion in [7]. Specifically, it has been shown in [7] that the optimal 70 frequency reuse factor is FR3 for the cell-edge users. The av71 erage cell throughput of an FFR system was derived in [8] as a 72 function of the distance threshold. It was shown in [9] that there 73 exists an optimal radius threshold for which the average rate be74 comes maximum. The performance of FFR and soft frequency 75 reuse (SFR) has been studied in [12] under both fully loaded 76 and partially loaded scenarios. An algorithm was proposed 77 in [13] for enhancing the network capacity and the cell-edge 78 performance for a dynamic SFR deployment relying on re79 alistic irregularly shaped cells. A fuzzy logic based generic 80 model was proposed for deriving different frequency reuse 81 schemes in [14]. As a further development, an FFR based 3-cell 82 network-MIMO based tri-sector BS architecture was presented 83 in [15]. FFR and SFR are compared in the presence of corre84 lated interferers in [16]. The optimal configuration of FFR is 85 determined in [17] for a high-density wireless cellular network. 86 The authors of [18] have proposed a distributed and adaptive 87 solution for interference coordination based on the center of 88 gravity of users in each sector. An optimal FFR and power 89 control scheme which can coordinate the interference among 90 the heterogeneous nodes is proposed in [19].

91 An analytical framework of calculating both the coverage 92 probability $\left(\mathrm{CP}_{r}\right)$ and the average rate of FFR schemes was 93 presented in [10] and [11] for homogeneous single input single 94 output (SISO) and MIMO heterogeneous networks, respec95 tively, using a Poisson point process (PPP). However, the au96 thors of [10], [11] assumed having an unplanned FFR network, 97 where the cells using the same frequency set are randomly 98 allocated. Hence, two cells using the same frequency for the 99 cell-edge users may in fact be co-located [10], [11]. However, 100 in case of FFR based deployments the regions using the same 101 frequency are typically planned to be as far apart as possible 102 and our focus is on these types of deployments. An FFR-aided 103 distributed antenna system (DAS) and an FFR-aided picocell 104 was studied in [20] and [21]. While, an FFR-aided femtocell 105 has been extensively studied in [22]-[26].

106 However, most of the work based on FFR has considered the 107 conventional SISO case. To the best of our knowledge, no prior 108 work has analytically derived the optimal SINR threshold for 109 FFR, when the number of antennas is high at the transmitter $110 \mathrm{and} /$ or at the receiver. Hence, in this work, we derive both the $111 \mathrm{CP}_{r}$ and the average achievable rate expressions of FFR in the 112 presence of both MU-MIMO as well as of SIMO systems and 113 derive the optimal SINR threshold corresponding to the desired $114 \mathrm{CP}_{r}$ and throughput. Furthermore, the performance of FFR115 aided MU-MIMOs is compared to that of FFR in the presence 116 of a SIMO system.

117 The key benefit of MU-MIMO is their ability to improve 118 the spectral efficiency, which has been extensively studied in 119 a single-cell context in the presence of AWGN [27]-[29]. 120 However, it has been shown in [30], [31] with the help of 121 simulation, that the efficiency of MU-MIMOs is significantly 122 eroded in a multi-cell environment due to interference, es123 pecially in the cell-edge region. FFR is capable of signifi124 cantly improving the cell-edge coverage since it uses FR3 for 125 the cell-edge users. Hence we study FFR-aided MU-MIMOs and quantify their average throughput as well as coverage 126 probability.

Furthermore, we carefully examine the correlation of the sub- 128 bands $F_{0}, F_{1}, F_{2}$ and $F_{3}$ in Fig. 1 used in the FFR system 129 considered. All prior work on FFR has assumed that the sub- 130 bands experience independent fading, which is mathematically 131 convenient, but practically not realisable. Indeed, when we 132 consider practical transmission block based modulation such as 133 OFDM, the channel's delay spread is assumed to be confined to 134 the cyclic prefix of the OFDM symbol. Such a limited-duration 135 (typically less than $20 \%$ of the useful OFDM symbol duration) 136 impulse response will result in correlation amongst the adjacent 137 frequency domain OFDM sub-channels. More explicitly, unless 138 the sub-bands $F_{0} \cdots F_{3}$ are spaced apart by more than the recip- 139 rocal of the delay spread, correlation will exist. Since the delay 140 spread experienced in the downlink is user-dependent, it is vir- 141 tually impossible to ensure that the sub-bands $F_{i}$ in Fig. 1 are in- 142 dependent for each user scheduled in the downlink. Therefore, 143 in our analysis we will specifically take into account the corre- 144 lation of the sub-bands. For FFR-aided MU-MIMO and SIMO 145 systems, the expressions of $\mathrm{CP}_{r}$ and average rate are derived 146 and the following new results are presented:

(a) The optimal SINR threshold that maximizes the $\mathrm{CP}_{r}$ of 148 FFR is derived for a given $T$. We show that the optimal 149 $S_{t h}$ (denoted by $S_{o p t, C}$ ) is $S_{t h}=T$ for both the MU-MIMO 150 and SIMO system, and if we choose the SINR threshold 151 to be $S_{\text {opt }, C}$, then the achievable $\mathrm{CP}_{r}$ of FFR is higher 152 than that of FR3. The improvement of the FFR $\mathrm{CP}_{r}$ over 153 that of FR3 is due to the resultant sub-band diversity gain 154 achieved by the systems when a user is classified as either 155 a cell-centre or a cell-edge user.

(b) The optimal SINR threshold that maximizes the average 157 rate of FFR is derived. We show that the optimal $S_{t h}$ (de- 158 noted by $S_{o p t, R}$ ) is equal to $T^{\prime}$ for both MU-MIMO and 159 SIMO systems, where $T^{\prime}$ is a fixed SINR value, which de- 160 pends on the system parameters such as the path loss expo- 161 nent, the number of antennas, the fading parameters, etc. 162

(c) The correlation of the sub-bands always degrades both the 163 $\mathrm{CP}_{r}$ and the average rate of the FFR-aided MU-MIMO 164 and SIMO systems.

165

(d) The performance of FFR-aided MU-MIMO and SIMO 166 systems is compared. It is shown that system designer 167 may choose the $(2 \times 2)$ MU-MIMO system over $(1 \times 3) 168$ SIMO system of FFR scheme as MU-MIMO achieves 169 significant gain in average rate over SIMO.

We will demonstrate that our analytical results are in close 171 agreement with the simulation results. Moreover, it is shown 172 that at optimal $S_{t h}$, the FFR achieves significantly high gain in 173 $\mathrm{CP}_{r}$ than that of average rate with respect to FR1 and hence this 174 scheme would be more useful when coverage gain is essentially 175 required. Therefore, FFR-aided MU-MIMO provides both high 176 average rate and satisfactory $\mathrm{CP}_{r}$ for a lower value of $N_{a}$.

\section{SYSTEM MODEL}

A homogeneous macrocell network relying on hexagonal 179 tessellation and on an inter cell site distance of $2 R$ is considered, 180 


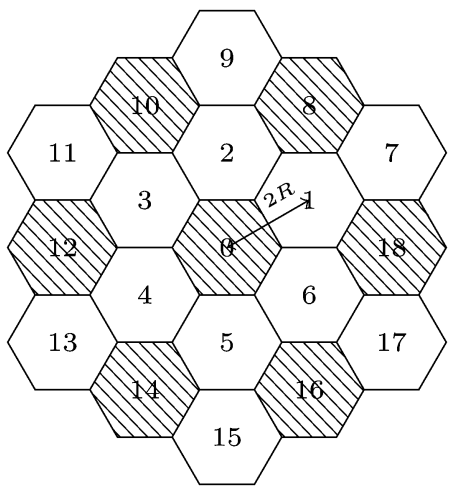

Fig. 2. Hexagonal structure of 2-tier macrocell. Interference for 0th cell in FR1 system is contributed form all the neighbouring 18 cells, while in a FR3 system it is contributed only from the shaded cells.

181 as shown in Fig. 2. Both a MU-MIMO and a SIMO system is 182 considered. We assume that in the MU-MIMO case each user 183 is equipped with $N_{r}$ receive antennas, while the BS is equipped 184 with $N_{t}$ transmit antennas and that $N_{t}=N_{r}$. Our focus is on the 185 downlink and hence $N_{t}$ transmit antennas are used for transmis186 sion, while the $N_{r}$ receive antennas at the UE are used for re187 ception. We also assume that all $N_{t}$ transmit antennas at the BS 188 are utilized to transmit $N_{t}$ independent data steams to its own $N_{t}$ 189 users. A linear minimum mean-square-error (LMMSE) receiver 190 [32] is considered. In order to calculate the post-processing 191 SINR of this LMMSE receiver, it is assumed that the $\left(N_{r}-1\right)$ 192 closest interferers can be completely cancelled using the anten193 nas at the receiver. ${ }^{3}$ For example, in the MU-MIMO case, the 194 user will not experience any intra-tier interference emanating 195 from the serving BS as $N_{t}=N_{r}$. In the SIMO case each user 196 is equipped with $N_{r}$ antennas. The SINR $\eta_{t}(r)$ of a user in the 197 MU-MIMO system and the SINR $\eta_{r}(r)$ of a user in the SIMO 198 system located at $r$ meters from its serving BS are given by

$$
\eta_{t}(r)=\frac{g r^{-\alpha}}{\frac{\sigma^{2}}{P}+I_{t}}, \quad I_{t}=\sum_{i \in \psi} \sum_{j=1}^{N_{t}} h_{i j} d_{i}^{-\alpha}
$$

199 and

$$
\eta_{r}(r)=\frac{g r^{-\alpha}}{\frac{\sigma^{2}}{P}+I_{r}}, \quad I_{r}=\sum_{i \in \psi_{r}} h_{i j} d_{i}^{-\alpha},
$$

200 respectively, where the transmit power of a BS is denoted by $P$. 201 Here $\psi$ is the set of interfering BSs in the FR1 network and $\psi_{r}$ 202 denotes all the interfering BSs, excluding the nearest $\left(N_{r}-1\right)$ 203 interferers, while $N_{t}$ denotes the number of transmit antennas. 204 The standard path loss model of $\|x\|^{-\alpha}$ is assumed, where $205 \alpha \geq 2$ is the path loss exponent and $\|x\|$ is the distance of a user 206 from the BS. We assumed that the users are at least at a distance 207 of $d$ away from the BS. ${ }^{4}$ The noise power is denoted by $\sigma^{2}$. 208 Here, $r$ and $d_{i}$ are the distances from the user to the serving BS 209 and to the $i^{\text {th }}$ interfering BS, respectively, while $g$ and $h_{i}$ denote

\footnotetext{
${ }^{3}$ It is widely exploited that using the LMMSE receiver $\left(N_{r}-1\right)$ interferers can be mitigated, where $N_{r}$ is the number of receive antennas [32]. However, for simplicity, we assume that the $N_{r}-1$ closest interferers can be completely cancelled.

${ }^{4}$ Typically, the path loss model is assumed to be $\max \{d,\|x\|\}^{-\alpha}$.
}

the corresponding channel fading power, which are independent 210 and identically exponentially distributed (i.i.d.) with a unit 211 mean, i.e., $g \sim \exp (1)$ and $h_{i} \sim \exp (1) \forall i$. In MU-MIMO case, 212 $h_{i j}$ is the channel's fading power from the $j^{\text {th }}$ antenna of the 213 $i^{t h}$ interfering BS to the user and it is i.i.d. with a unit mean. 214 Without loss of generality we have considered a user in the $0^{\text {th }} 215$ cell of Fig. 2 in our analysis.

Similar to [10], the subscribers are classified as cell-centre 217 users and cell-edge users based on the SINR at the mobile sta- 218 tion. If the calculated SINR of a user is lower than the specified 219 SINR threshold $S_{t h}$, the user is classified as a cell-edge user. 220 Otherwise, the user is classified as a cell-centre user. Typically, 221 FFR divides the whole frequency band into a total of $(1+\delta) 222$ parts, where $F_{0}$ is allocated to all the cells for the cell-centre 223 users, as seen in Fig. 1. One of the $\{1, \cdots, \delta\}$ parts is assigned 224 to the cell-edge users in each cell in a planned fashion. The 225 users are assumed to be uniformly distributed in a cell and all re- 226 source blocks are uniformly shared among the users. The trans- 227 mit power is assumed to be fixed. If we have $\eta_{t}(r)\left(\right.$ or $\left.\eta_{r}(r)\right) \geq 228$ $S_{t h}$ for a user, then the user will continue to experience the same 229 fading power, i.e., $g$ and $h_{i}$ from the user to the serving BS 230 and to the $i^{\text {th }}$ interfering BS, respectively. However, if we have 231 $\eta_{t}(r)$ (or $\left.\eta_{r}(r)\right)<S_{t h}$ for a user, the user is allocated another 232 sub-band (from the set of sub-bands assigned to cell-edge users) 233 and it experiences a new fading power, i.e., $\hat{g}$ and $\hat{h}_{i}$ from the 234 user to the serving BS and to the $i^{\text {th }}$ interfering BS, respectively. 235 Based on the coherence bandwidth of the OFDM system, and 236 the bands associated with $F_{0}$ to $F_{3}$ in Fig. 1 is is possible that $\hat{g} 237$ and $\hat{h}_{i}$ are either correlated with or independent of $g$ and $h_{i}$, re- 238 spectively. Note that $g, \hat{g}, h_{i}$, and $\hat{h}_{i}$ are the channel gains in the 239 frequency domain and the term correlation is used for referring 240 to frequency domain correlation in this paper. The correlation 241 depends both on the particular user's channel conditions and 242 on the instantaneous coherence bandwidth with respect to the 243 FFR frequency bands. To better understand the impact of corre- 244 lation among the sub-bands on the FFR system's performance, 245 in this paper, we consider the following two extreme cases: 246

Case 1: $g$ and $\hat{g}$ are independent and also $h_{i}$ as well as $\hat{h}_{i}$, are 247 independent for all $i$.

248

Case 2: $g$ and $\hat{g}$ are fully correlated and also $h_{i}$ as well as $\hat{h}_{i}, 249$ are fully correlated for all $i$.

In reality these channel output powers may be partially corre- 251 lated, but the analysis of partial (arbitrary) correlation is quite 252 complicated and hence it is beyond the scope of this work. 253 However, the analysis of the above two extreme cases we be- 254 lieve, is sufficient for understanding the impact of correlation 255 among the sub-bands.

\section{Coverage Probability Analysis of FFR}

In this section, we first derive the $\mathrm{CP}_{r}$ of both the 258 MU-MIMO and SIMO system considered, which is defined 259 as the probability that a randomly chosen user's instantaneous 260 SINR $\eta_{t}(r)$ is higher than $T$. This defines, the average fraction 261 of users are having an SINR higher than the target SINR. The 262 coverage probability is determined by the complementry cumu- 263 lative distribution function of the SINR over the network. The 264 
$265 \mathrm{CP}_{r}$ of a user who is at a distance of $r$ meters from the $\mathrm{BS}$ in a 266 FR1-aided MU-MIMO scenario is given by

$$
P_{1}(T, r)=P\left[\eta_{t}(r)>T\right]=P\left[g>\operatorname{Tr}^{\alpha} I_{t}+\operatorname{Tr}^{\alpha} \frac{\sigma^{2}}{P}\right],
$$

267 where $I_{t}$ is defined in (2). Since $g \sim \exp (1), h_{i j} \sim \exp (1)$, and $268 h_{i j}$ are i.i.d., $P_{1}(T, r)$ is given by

$$
\begin{gathered}
P_{1}(T, r)=E_{h_{i j}}\left[e^{-\operatorname{Tr}^{\alpha} I_{t}-T^{\alpha} \frac{\sigma^{2}}{P}}\right]=\prod_{i \in \psi} \prod_{j=1}^{N_{t}} E_{h_{i j}}\left[e^{-T^{\alpha} h_{i j} d_{i}^{-\alpha}}\right] \\
\times e^{-T^{\alpha} \frac{\sigma^{2}}{P}}=\prod_{i \in \psi}\left(\frac{1}{1+\operatorname{Tr}^{\alpha} d_{i}^{-\alpha}}\right)^{N_{t}} e^{-\operatorname{Tr}^{\alpha} \frac{\sigma^{2}}{P}}
\end{gathered}
$$

269 where $\psi$ is the set of interfering BSs in a FR1 network. 270 Similarly, the $\mathrm{CP}_{r}$ of a user located at a distance of $r$ meters 271 from the BS in a FR3 network can be formulated as

$$
P_{3}(T, r)=\prod_{i \in \phi}\left(\frac{1}{1+T r^{\alpha} d_{i}^{-\alpha}}\right)^{N_{t}} e^{-T r^{\alpha} \frac{\sigma^{2}}{P}}
$$

272 where $\phi$ is the set of interfering cells in the FR3 scheme, which 273 is a function of the frequency reuse plan. Also, the $\mathrm{CP}_{r}$ of a user 274 in the SIMO-based FR1 network and in a FR3 network can be 275 expressed as

$$
\begin{aligned}
& P_{1}(T, r)=\prod_{i \in \psi_{r}} \frac{1}{1+T r^{\alpha} d_{i}^{-\alpha}} e^{-T r^{\alpha} \frac{\sigma^{2}}{P}} \quad \text { and } \\
& P_{3}(T, r)=\prod_{i \in \phi_{r}} \frac{1}{1+T r^{\alpha} d_{i}^{-\alpha}} e^{-T r^{\alpha} \frac{\sigma^{2}}{P}} .
\end{aligned}
$$

276 Here $\phi_{r}$ denotes the set of interfering cells in the FR3 scheme 277 excluding the nearest $\left(N_{r}-1\right)$ interferers. Let us now derive 278 the $\mathrm{CP}_{r}$ of FFR for both the independent and correlated cases.

279 A. Case 1: $g$ and $\hat{g}$ are Independent as Well as $h_{i}$ and $\hat{h}_{i}$ are 280 Also Independent for all $i$

281 The $\mathrm{CP}_{r} P_{F, c}(r)$ of a cell-centre user who is at a distance of $282 r$ meters from the $0^{\text {th }}$ BS in a FFR-aided MU-MIMO scenario 283 is given by

$$
\begin{aligned}
P_{F, c}(r) & \stackrel{(a)}{=} P\left[\eta_{t}(r)>T \mid \eta_{t}(r)>S_{t h}\right] \\
& =P\left[\frac{g r^{-\alpha}}{I_{t}+\frac{\sigma^{2}}{P}}>T \mid \frac{g r^{-\alpha}}{I_{t}+\frac{\sigma^{2}}{P}}>S_{t h}\right],
\end{aligned}
$$

284 where $(a)$ follows from the fact that a cell-centre user has SINR $285 \geq S_{t h}$. Upon applying Bayes' rule, one can rewrite $P_{F, c}(r)$ as

$$
\begin{aligned}
P_{F, c}(r) & =\frac{P\left[\frac{g r^{-\alpha}}{I_{t}+\frac{\sigma^{2}}{P}}>T, \frac{g r^{-\alpha}}{I_{t}+\frac{\sigma^{2}}{P}}>S_{t h}\right]}{P\left[\frac{g r^{-\alpha}}{I_{t}+\frac{\sigma^{2}}{P}}>S_{t h}\right]} \\
= & \frac{\prod_{i \in \psi}\left(\frac{1}{1+\max \left\{T, S_{t h}\right\} r^{\alpha} d_{i}^{-\alpha}}\right)^{N_{t}} e^{-\max \left\{T, S_{t h}\right\} r^{\alpha} \frac{\sigma^{2}}{P}}}{\prod_{j \in \psi}\left(\frac{1}{1+S_{t h} r^{\alpha} d_{j}^{-\alpha}}\right)^{N_{t}} e^{-S_{t h} r^{\alpha} \frac{\sigma^{2}}{P}}} .
\end{aligned}
$$

Similarly, the $\mathrm{CP}_{r}$ of a cell-edge user who is at a distance of $r 286$ meters from the BS in the FFR-aided MU-MIMO case $P_{F, e}(r) 287$ is given by

$$
\begin{aligned}
P_{F, e}(r) & =P\left[\hat{\eta}_{t}(r)>T \mid \eta_{t}(r)<S_{t h}\right] \\
& =\frac{P\left[\frac{\hat{g} r^{-\alpha}}{\hat{I}_{t}+\frac{\sigma^{2}}{P}}>T, \frac{g r^{-\alpha}}{I_{t}+\frac{\sigma^{2}}{P}}<S_{t h}\right]}{P\left[\frac{g r^{-\alpha}}{I_{t}+\frac{\sigma^{2}}{P}}<S_{t h}\right]} .
\end{aligned}
$$

Here, the cell-edge user will experience the new interference 289 term of $\hat{I}_{t}=\sum_{i \in \phi} \sum_{j=1}^{N_{t}} \hat{h}_{i j} d_{i}^{-\alpha}$ and the new channel power $\hat{g}$, i.e. a 290 new SINR $\hat{\eta}(r)$ due to the fact that the cell-edge user is now a 291 FR3 user. Basically, $\hat{\eta}(r)$ denotes the SINR experienced by the 292 user at a distance of $r$ meters from the BS in a FR3 system and 293 is given by

$$
\hat{\eta}(r)=\frac{\hat{g} r^{-\alpha}}{\hat{I}_{t}+\frac{\sigma^{2}}{P}}, \quad \hat{I}_{t}=\sum_{i \in \phi} \sum_{j=1}^{N_{t}} \hat{h}_{i j} d_{i}^{-\alpha} .
$$

Since both $g$ and $\hat{g}$ as well as $h_{i}$ and $\hat{h}_{i}$ are assumed to be i.i.d, 295 $P_{F, e}(r)$ can be simplified to

$$
P_{F, e}(r)=P\left[\frac{\hat{g} r^{-\alpha}}{\hat{I}_{t}+\frac{\sigma^{2}}{P}}>T\right]=P_{3}(T, r) .
$$

Let us now derive the $\mathrm{CP}_{r} P_{f}(r)$ of a user in the FFR-aided 297 MU-MIMO system, which can be written as

$$
P_{F}(r)=P_{F, c}(r) P\left[\eta_{t}(r)>S_{t h}\right]+P_{F, e}(r) P\left[\eta_{t}(r)<S_{t h}\right] .
$$

Here, the first term denotes the $\mathrm{CP}_{r}$ contributed by the cell- 299 centre users, while the second term denotes the contribution of 300 the cell-edge users. By using the expression in (7) for $P_{F, c}(r) 301$ and the expression in (9) for $P_{F, e}(r),(10)$ can be simpli- 302 fied to

$$
\begin{array}{r}
P_{F}(r)=\prod_{i \in \psi}\left(\frac{1}{1+\max \left\{T, S_{t h}\right\} r^{\alpha} d_{i}^{-\alpha}}\right)^{N_{t}} e^{-\max \left\{T, S_{t h}\right\} r^{\alpha} \frac{\sigma^{2}}{P}} \\
+P_{3}(T, r)-P_{3}(T, r) P_{1}\left(S_{t h}, r\right) .
\end{array}
$$

Lemma 1: The optimum $S_{\text {th }}$ (denoted by $S_{\text {opt }, C}$ ) that maxi- 304 mizes the FFR-aided coverage probability is $S_{t h}=T$, and when 305 the SINR threshold is set to $S_{\text {opt }, c}$, the coverage probability of 306 FFR becomes higher than that of FR3.

Proof: See Appendix A for the proof.

B. Case 2: $g$ and $\hat{g}$ are Completely Correlated as Well as $h_{i} \quad 309$ and $\hat{h}_{i}$ are Also Completely Correlated for all $i$

Note that the centre $\mathrm{CP}_{r}$ is the same for both the above 311 Case 1 and for this case, since a user does not change its sub- 312 band, when it becomes a cell-centre user because if $\eta_{t}(r) \geq S_{t h} 313$ for a user, then it will continue to experience the same fading 314 power. However, the edge $\mathrm{CP}_{r}$ is different in Case 1 as well as 315 Case 2, and in this scenario the $\mathrm{CP}_{r} P_{F, e}(r)$ of a cell-edge user, 316 
317 who is at a distance of $r$ meters from the BS in our FFR network 318 is given by

$$
P_{F, e}(r)=P\left[\hat{\eta}_{t}(r)>T \mid \eta_{t}(r)<S_{t h}\right]=\frac{P\left[\hat{\eta}_{t}(r)>T, \eta_{t}(r)<S_{t h}\right]}{P\left[\eta_{t}(r)<S_{t h}\right]} .
$$

319 Substituting the value of $P_{F, c}$ and $P_{F, e}$ from (7) and (12) into 320 Eq. (10), the $\mathrm{CP}_{r} P_{f}(r)$ in our FFR network can be written as

$$
\begin{array}{r}
P_{F}(r)=\prod_{i \in \psi}\left(\frac{1}{1+\max \left\{T, S_{t h}\right\} r^{\alpha} d_{i}^{-\alpha}}\right)^{N_{t}} e^{-\max \left\{T, S_{t h}\right\} r^{\alpha} \frac{\sigma^{2}}{P}} \\
+P\left[\hat{\eta}_{t}(r)>T, \eta_{t}(r)<S_{t h}\right] .
\end{array}
$$

321 Recall that $\eta_{t}(r)$ and $\hat{\eta}_{t}(r)$ represent the SINR experienced by a 322 user in an FR1 and an FR3 system, respectively. Note that even 323 though $g$ and $\hat{g}$ as well as $h_{i}$ and $\hat{h}_{i}$ are completely correlated, $324 \eta_{t}(r)$ is not the same as $\hat{\eta}_{t}(r)$, because the set of interferers are 325 different in the denominator of the $\eta_{t}(r)$ and $\hat{\eta}_{t}(r)$ expressions 326 given in (2) and (8), respectively, i.e., $\psi$ corresponds to the 327 set of interferers in the FR1 network, while $\phi$ corresponds to 328 the set of interferers in the FR3 network. Since $g$ and $\hat{g}$ are 329 completely correlated and $h_{i}$ and $\hat{h}_{i}$ are also completely corre330 lated for all $i$, we use the following transformation to further 331 simplify $P_{F}(r)$ :

$$
P\left[\hat{\eta}_{t}(r)>T, \eta_{t}(r)<S_{t h}\right]=P\left[\hat{\eta}_{t}(r)>T, \hat{\eta}_{t}(r)<\hat{S}_{t h}\right] .
$$

332 Basically instead of marking a user as a cell-edge user based 333 on the FR1 SINR $\eta_{t}(r)$, we mark them on the basis of the FR3 334 SINR $\hat{\eta}_{t}(r)$ by introducing a new SINR threshold $\hat{S}_{t h}$. In other 335 words, we introduce a new SINR threshold $\hat{S}_{t h}$ for ensuring that 336 if for any user we have $\eta_{t}(r)<S_{t h}$, then for the same user we 337 have $\hat{\eta}_{t}(r)<\hat{S}_{t h}$ and vice-versa. The threshold $\hat{S}_{t h}$ is computed 338 using the relationship of $P\left[\eta_{t}(r)<S_{t h}\right]=P\left[\hat{\eta}_{t}(r)<\hat{S}_{t h}\right]$. This 339 ensures that the same user is marked as a cell-edge user for both 340 reuse patterns FR1 and FR3. Now, using the transformation 341 given in $(14), P_{F}(r)$ can be simplified to

$$
\begin{aligned}
P_{F}(r)=\prod_{i \in \psi} & \left(\frac{1}{1+\max \left\{T, S_{t h}\right\} r^{\alpha} d_{i}^{-\alpha}}\right)^{N_{t}} e^{-\max \left\{T, S_{t h}\right\} r^{\alpha} \frac{\sigma^{2}}{P}} \\
& +P[\hat{\eta}(r)>T]-P\left[\hat{\eta}(r)>\max \left\{\hat{S}_{t h}, T\right\}\right] .
\end{aligned}
$$

342 In this case, to obtain the optimum $S_{\text {opt }, C}$, we consider the 343 following two possibilities: (i) $S_{t h} \geq T$, (ii) $S_{t h}<T$.

(i) $S_{t h} \geq T$ : In this scenario, $C P_{f}(r)$ can be expressed in terms of $T$ as:

$$
\begin{array}{r}
P_{F}\left(r, S_{t h} \geq T\right)=\prod_{i \in \psi} \frac{1}{1+} S_{t h} r^{\alpha} d_{i}^{-\alpha} e^{-S_{t h} r^{\alpha} \frac{\sigma^{2}}{P}} \\
+P_{3}(T, r)-P_{3}\left(\hat{S}_{t h}, r\right) .
\end{array}
$$

346 Since we have $P_{3}\left(\hat{S}_{t h}, r\right)=P_{1}\left(S_{t h}, r\right)$ and $P_{1}\left(S_{t h}, r\right)=$ 347

$$
\begin{aligned}
& \prod_{i \in \psi}\left(\frac{1}{1+S_{t h} r^{\alpha} d_{i}^{-\alpha}}\right)^{N_{t}} e^{-S_{t h} r^{\alpha} \frac{\sigma^{2}}{P}} \text {, hence } \\
& P_{F}\left(r, S_{t h} \geq T\right)=P_{3}(T, r) .
\end{aligned}
$$

(ii) $S_{t h}<T$ : In this case $P_{f}(r)$ can be formulated in terms 348 of $T$ as:

$$
\begin{aligned}
P_{F}\left(r, S_{t h}<T\right) & =\prod_{i \in \psi}\left(\frac{1}{1+\operatorname{Tr}^{\alpha} d_{i}^{-\alpha}}\right)^{N_{t}} e^{-T^{\alpha} \frac{\sigma^{2}}{P}} \\
& +P_{3}(T, r)-P_{3}\left(\max \left\{\hat{S}_{t h}, T\right\}, r\right) .
\end{aligned}
$$

Note that when $S_{t h}<T, \hat{S}_{t h}$ may be higher or lower than $T .350$ When $\hat{S}_{t h}>T$,

$$
P_{3}\left(\max \left\{\hat{S}_{t h}, T\right\}, r\right)=P_{3}\left(\hat{S}_{t h}, r\right)=P_{1}\left(S_{t h}, r\right)>P_{1}(T, r)
$$

since $S_{t h}<T$. And when $\hat{S}_{t h}<T$, we have:

$$
P_{3}\left(\max \left\{\hat{S}_{t h}, T\right\}, r\right)=P_{3}(T, r)>P_{1}(T, r) .
$$

Hence, we arrive at:

$$
\begin{aligned}
P_{F}\left(r, S_{t h}\right. & <T)=\prod_{i \in \psi}\left(\frac{1}{1+\operatorname{Tr}^{\alpha} d_{i}^{-\alpha}}\right)^{N_{t}} e^{-T r^{\alpha} \frac{\sigma^{2}}{P}} \\
& +P_{3}(T, r)-P_{3}\left(\max \left\{\hat{S}_{t h}, T\right\}, r\right)<P_{3}(T, r) .
\end{aligned}
$$

Comparing the FFR $\mathrm{CP}_{r}$ for $S_{t h} \geq T$ and $S_{t h}<T$ given by (17) 354 and (21), respectively, it becomes apparent that $P_{F}\left(r, S_{t h} \geq 355\right.$ $T)>P_{F}\left(r, S_{t h}<T\right)$. In other words, when the fading is fully 356 correlated across the sub-bands, the optimal choice of the SINR 357 threshold is $S_{t h} \geq T$ and at the optimal SINR threshold the FFR 358 scheme succeeds in achieving the $\mathrm{FR} 3 \mathrm{CP}_{r}$. Unlike for Case 1, 359 the FFR $\mathrm{CP}_{r}$ is not better than the $\mathrm{FR} 3 \mathrm{CP}_{r}$ since there is no sub- 360 band diversity gain, when a user moves from the cell-centre to 361 the cell-edge region.

362

In order to find the $\mathrm{CP}_{r}$ for a typical user, we have to calculate 363 the probability density function (pdf) of $r$, which is the distance 364 between the $0^{\text {th }}$ BS (serving BS) and the desired user. To 365 calculate this pdf, we model the cell shape by an inner circle 366 within a hexagonal cell [33], and assume that the users are 367 uniformly distributed. Therefore, the pdf $f_{R}(r)$ of $r$ is given by 368

$$
\begin{aligned}
& f_{R}(r)=\left\{\begin{array}{c}
\frac{2 r}{R^{2}}, r \leqslant R \\
0, r>R
\end{array}\right. \\
& \text { IV. AVERAGE RATE }
\end{aligned}
$$

In this section, we derive the average rate of both the FFR- 370 aided MU-MIMO as well as of its SIMO counterpart and find 371 the optimum value of $S_{t h}$ (denoted by $S_{\text {opt }, R}$ ) for which the 372 average rate is maximum. The average rate of the system is 373 given by $R=E[\ln (1+\mathrm{SINR})]$. In order to derive the average 374 rate $^{5}$ for the FFR system, we have to consider its sub-band al- 375 location. Since the users are uniformly distributed, the specific 376 sub-band allocated to the cell-centre users and cell-edge users 377 are given by [9], [10] $N_{c}=N_{t} P_{F, c}$ and $N_{e}=\frac{N_{t}-N_{c}}{3}$, where $P_{F, c} 378$ denotes the specific fraction of cell-centre users, while $N_{t}, N_{c} 379$ and $N_{e}$ denote the total band, cell-centre sub-band and cell-edge 380

\footnotetext{
${ }^{5}$ An interference limited system is assumed for simplicity, which implies ignoring the effects of noise. However, the derivation of the average rate can be readily extended to the case, where the thermal noise is also considered.
} 
381 sub-band, respectively. Let us now derive the average rate for 382 the planned FFR-aided MU-MIMO case.

\section{A. Average Rate in the FRI and FR3 Systems}

384 The average rate of a user at a distance $r$ is $E\left[\ln \left(1+\eta_{t}(r)\right)\right]$. 385 By exploiting the fact that for a positive random variable $X=$ $386 \ln \left(1+\eta_{t}(r)\right)$ we have $E[X]=\int_{t>0} P(X>t) \mathrm{d} t$, the rate $R_{1}(r)$ 387 can be rewritten as

$$
\begin{aligned}
R_{1}(r) & =\int_{t>0} P\left[\ln \left(1+\eta_{t}(r)\right)>t\right] \mathrm{d} t=\int_{t>0} P\left[\eta_{t}(r)>e^{t}-1\right] \mathrm{d} t \\
& =\int_{t>0} \prod_{j \in \psi}\left(\frac{1}{1+\left(e^{t}-1\right) r^{\alpha} d_{j}^{-\alpha}}\right)^{N_{t}} \mathrm{~d} t
\end{aligned}
$$

388 which follows from (3) and (4). Let us now determine the 389 average rate of the FR1 system, where spatially averaged rate $390 R_{1}$ can be expressed as

$$
R_{1}=\int_{0}^{R} \int_{t>0} \prod_{j \in \psi}\left(\frac{1}{1+\left(e^{t}-1\right) r^{\alpha} d_{j}^{-\alpha}}\right)^{N_{t}} \mathrm{~d} t f_{R}(r) \mathrm{d} r .
$$

391 The average rate of FR3 can be obtained in a similar fashion, 392 which is given by

$$
R_{3}=\int_{0}^{R} \int_{t>0} \prod_{i \in \phi}\left(\frac{1}{1+\left(e^{t}-1\right) r^{\alpha} d_{i}^{-\alpha}}\right)^{N_{t}} \mathrm{~d} t f_{R}(r) \mathrm{d} r .
$$

393 B. Average Rate of the FFR System, When the 394 Sub-Bands are Independent

395 Lemma 2: The average rate of the FFR-aided MU-MIMO 396 system is given by

$$
\begin{aligned}
R_{f}=\int_{0}^{R} \int_{t>0}\left(\prod_{j \in \psi}\left(\frac{1}{1+\max \left\{e^{t}-1, S_{t h}\right\} r^{\alpha} d_{j}^{-\alpha}}\right)^{N_{t}}\right. \\
\left.+\frac{1}{3} \prod_{i \in \phi} \frac{P\left[\eta_{t}(r)<S_{t h}\right]}{\left(1+\left(e^{t}-1\right) r^{\alpha} d_{i}^{-\alpha}\right)^{N_{t}}}\right) \mathrm{d} t f_{R}(r) \mathrm{d} r .
\end{aligned}
$$

Proof: See Appendix B for the proof.

Similarly, the average rate of the FFR-aided SIMO system is 398 given by

$$
\begin{aligned}
R_{f}=\int_{0}^{R} \int_{t>0}\left(\prod_{j \in \psi_{r}}\right. & \frac{1}{1+\max \left\{e^{t}-1, S_{t h}\right\} r^{\alpha} d_{j}^{-\alpha}} \\
& \left.+\frac{1}{3} \prod_{i \in \phi_{r}} \frac{P\left[\eta_{r}(r)<S_{t h}\right]}{1+\left(e^{t}-1\right) r^{\alpha} d_{i}^{-\alpha}}\right) \mathrm{d} t f_{R}(r) \mathrm{d} r
\end{aligned}
$$

\section{Optimum Value of the SIR Threshold $S_{\text {opt }, R}$, When the Sub-Bands are Independent}

The optimum value of $S_{t h}$ (denoted by $S_{o p t, R}$ ) for which the 402 average rate of the FFR system is maximized is derived and it 403 is shown to be a function of both the number of antennas and of 404 the path loss exponent.

405

Lemma 3: The value of $S_{t h}$ which maximizes the average rate 406 of the FFR system is $S_{o p t, R}=T^{\prime}$, where $T^{\prime}$ can be obtained as 407 the solution of equation given in (28), shown at the bottom of 408 the page, where, $K(r)$ is defined later in (47).

409

Proof: See Appendix C for the proof.

Note that the optimal $S_{t h}$ of the SIMO scenario can be derived 411 by following the method of the MU-MIMO case and it is 412 $S_{\text {opt }, R}=T^{\prime}$, where $T^{\prime}$ can be obtained as the solution of the 413 equation given in (29), shown at the bottom of the page, where 414 we have $K(r)=\frac{1}{3} \int \prod_{t>0} \frac{1}{1+\left(e^{t}-1\right) r^{\alpha} d_{i}^{-\alpha}} \mathrm{d} t$.

Fig. 3 plots the optimal SINR threshold $S_{t h}$ versus the number 416 of antennas for different path loss exponent. It can be observed 417 for the MU-MIMO case that as the number of transmit antennas 418 is reduced, $S_{\text {opt }, R}$ increases. Intuitively, as the number of trans- 419 mit antennas decreases, the interference experienced by the user 420 would decrease as the interference from the other cell decrease. 421 Thus, the average SINR of all users increases. Hence, the opti- 422 mal SINR threshold increases in order to balance the ratio of 423 cell-edge users and cell-centre users. Similarly, as the number 424 of receive antennas increases, the average SINR increases in 425 SIMO scenario, because more antennas are capable of can- 426 celling more of the closest interferers. Hence, $S_{o p t, R}$ increases 427

$$
\int_{0}^{R}\left(\frac{\left(K(r)-\ln \left(1+T^{\prime}\right)\right) \sum_{i \in \psi}\left(1+T^{\prime} r^{\alpha} d_{i}^{-\alpha}\right)^{N_{t}-1} r^{\alpha} d_{i}^{-\alpha}\left(\prod_{j \in \psi \backslash i}\left(1+T^{\prime} r^{\alpha} d_{j}^{-\alpha}\right)^{N_{t}}\right)}{\left(\prod_{j \in \psi}\left(1+T^{\prime} r^{\alpha} d_{j}^{-\alpha}\right)\right)^{2 N_{t}}}\right) f_{R}(r) \mathrm{d} r=0,
$$

$$
\int_{0}^{R}\left(\frac{\left(K(r)-\ln \left(1+T^{\prime}\right)\right) \sum_{i \in \psi_{r}} r^{\alpha} d_{i}^{-\alpha}\left(\prod_{j \in \psi_{r} \backslash i}\left(1+T^{\prime} r^{\alpha} d_{j}^{-\alpha}\right)\right)}{\left(\prod_{j \in \psi_{r}}\left(1+T^{\prime} r^{\alpha} d_{j}^{-\alpha}\right)\right)^{2}}\right) f_{R}(r) \mathrm{d} r=0,
$$




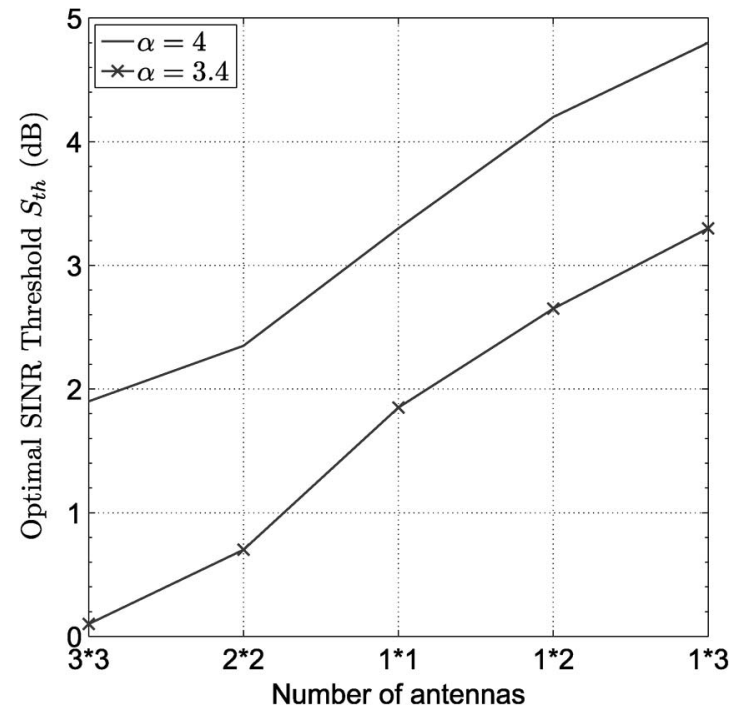

Fig. 3. Optimal SINR threshold $S_{t h}$ evaluated using (28) and (29) versus the number of antennas for different path-loss exponents.

428 in order to balance the ratio of cell-centre users and cell-edge 429 users. Furthermore, as the path loss exponent decreases, the 430 average SIR of all the users decreases and hence $S_{\text {opt }, R}$ 431 decreases.

432 D. Average Rate of the FFR System, When the Sub-Bands are 433 Completely Correlated

434 In this subsection first we derive the average rate $R_{f}(r)$ of the 435 FFR system for the MU-MIMO case. The average rate of the 436 FFR system given in (39) can be rewritten as

$$
R_{f}(r)=R_{c}(r) P\left[\eta_{t}(r)>S_{t h}\right]+\frac{1}{3} R_{e}(r) P\left[\eta_{t}(r)<S_{t h}\right] .
$$

437 Note that the first term $R_{c}(r) P\left[\eta_{t}(r)>S_{t h}\right]$ denotes the average 438 rate contributed by the cell-centre users and it is the same 439 regardless, whether the fading of the bands is correlated or inde440 pendent across the sub-bands. Similar to the average rate of the 441 FFR system given in (39), the factor $\frac{1}{3}$ is introduced in the sec442 ond term, since a frequency reuse factor of $\frac{1}{3}$ is invoked for the 443 cell-edge users. In other words, only one third of the cell-edge 444 frequency $\left(F_{1}+F_{2}+F_{3}\right)$ is used for the cell-edge users and 445 hence the factor $\frac{1}{3}$ multiplies the second term of (30). Now, us446 ing the expression of $R_{e}(r)$ in $(42), R_{e}(r) P\left[\eta_{t}(r)<S_{t h}\right]$ can be 447 written as

$$
R_{e}(r) P\left[\eta_{t}(r)<S_{t h}\right]=\int_{t>0} P\left[\hat{\eta}_{t}(r)>e^{t}-1, \eta_{t}(r)<S_{t h}\right] \mathrm{dt} .
$$

448 Using the transformation in (14), $R_{e}(r) P\left[\eta_{t}(r)<S_{t h}\right]$ can be 449 simplified to

$$
\begin{aligned}
R_{e}(r) P\left[\eta_{t}(r)<S_{t h}\right] & =\int_{t>0} P\left[\hat{\eta}_{t}(r)>e^{t}-1\right] \\
& -P\left[\hat{\eta}_{t}(r)>\max \left\{e^{t}-1, \hat{S}_{t h}\right\}\right] \mathrm{dt} .
\end{aligned}
$$

Using the result of (25), $R_{e}(r) P\left[\eta(r)<S_{t h}\right]$ can be further 450 simplified to

$$
\begin{aligned}
R_{e}(r) P\left[\eta_{t}(r)<S_{t h}\right] & =\int_{t>0} \prod_{i \in \phi} \frac{1}{1+\left(e^{t}-1\right) r^{\alpha} d_{i}^{-\alpha}} \\
& -\prod_{i \in \phi} \frac{1}{1+\max \left\{e^{t}-1, \hat{S}_{t h}\right\} r^{\alpha} d_{i}^{-\alpha}} \mathrm{dt} .
\end{aligned}
$$

Finally, substituting back (41) as well as (33) into (30) and then 452 averaging over the spatial dimension, the average rate of the 453 FFR system is given as

$$
\begin{gathered}
R_{f}=\int_{0}^{R} \int_{t>0} \prod_{j \in \psi} \frac{1}{1+\max \left\{e^{t}-1, S_{t h}\right\} r^{\alpha} d_{j}^{-\alpha}}+\frac{1}{3}\left(\prod_{i \in \phi} \frac{1}{1+\left(e^{t}-1\right) r^{\alpha} d_{i}^{-\alpha}}\right. \\
\left.-\prod_{i \in \phi} \frac{1}{1+\max \left\{e^{t}-1, \hat{S}_{t h}\right\} r^{\alpha} d_{i}^{-\alpha}}\right) \mathrm{d} t f_{R}(r) \mathrm{d} r . \quad \text { (34) } \\
\text { V. Simulation Results }
\end{gathered}
$$

In this section, we provide the simulation results in order to 456 verify our analytical results In the simulations, we have con- 457 sidered the classic 19 cell system associated with a hexagonal 458 structure having a radius of 1000 meters. A LTE system having 459 a $10 \mathrm{MHz}$ bandwidth, 50 physical resource blocks (PRB) and 460 25 users is considered for each cell. The users are assumed to be 461 uniformly distributed in a cell and similarly, all resource blocks 462 are uniformly shared among users. In other words, if there are 463 $K$ users and $R$ resource blocks then each user is assigned $\frac{R}{K}$ re- 464 source blocks. For each user we generate the channel fading 465 power corresponding to its own channel as well as that corre- 466 sponding to the 18 interferers and then compute the SIR per user 467 per PRB. If a user having an SIR higher than $S_{\text {th }}$ over 25 or more 468 than 25 PRBs, then the user is considered to be a cell-centre 469 user, otherwise it is classified as a cell-edge user. For the 470 analytical $\mathrm{CP}_{r}$ computation, (11) and (15) are used for the inde- 471 pendent and correlated cases, respectively. Fig. 4 shows the 472 variation of $\mathrm{CP}_{r}$ as a function of the SINR threshold for FR1, 473 FR3, and the FFR case using both our analytical expressions in 474 (11) and (15) and simulations. Observe in Fig. 4 that the ana- 475 lytical results match the simulation results. It can be seen that 476 for the independent fading case, the $\mathrm{CP}_{r}$ reaches its maximum, 477 when $S_{t h}=T$ and it becomes higher than the FR3 $\mathrm{CP}_{r}$. How- 478 ever, for the fully correlated case, the $\mathrm{CP}_{r}$ becomes maximum, 479 when $S_{t h} \geq T$ and it is equal to the $\mathrm{FR} 3 \mathrm{CP}_{r}$.

Note that all our results are based on considering Rayleigh 481 fading. However, the results seem to be valid for general fading. 482 For example, Fig. 5 shows the variation of $C P_{r}$ as a function 483 of the SINR threshold by considering Nakagami-m fading 484 using simulations. The $C P_{r}$ is shown for the FR1, FR3 and 485 FFR scenarios for the different values of the Nakagami shape 486 parameter $m$. Similar to the Rayleigh fading scenario, the $C P_{r} 487$ reaches its maximum, when $S_{t h}=T$ and it becomes higher than 488 the FR3 $C P_{r}$. Interestingly, as the Nakagami shape parameter 489 increases, the gap between the optimal FFR $C P_{r}$ and FR3 $C P_{r} 490$ 


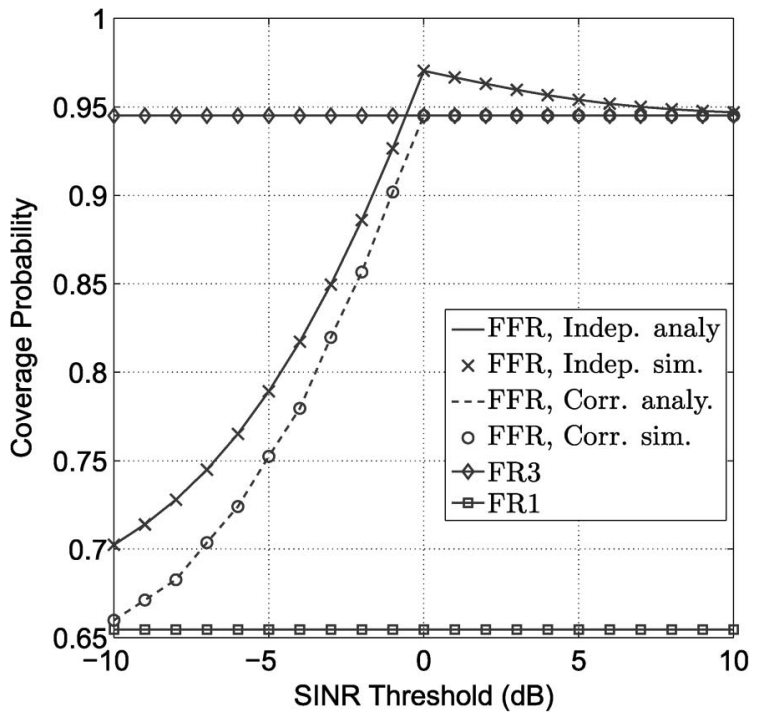

Fig. 4. Coverage probability of FR1, FR3 and FFR evaluated for (11) and (15) with respect to SINR Threshold $S_{t h}$. Here, $T=0 \mathrm{~dB}, \alpha=3.2$ and $N_{t}=N_{r}=1$.

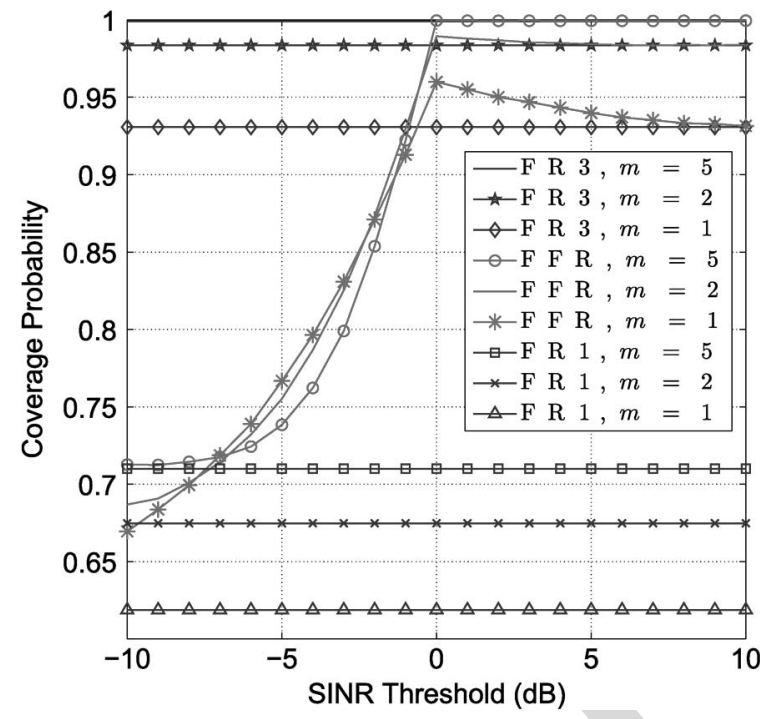

Fig. 5. Coverage probability of FR1, FR3 and FFR for different value of shape parameter for Nakagami-m fading. Here, $T=0 \mathrm{~dB}, \alpha=3$ and $N_{t}=N_{r}=1$.

491 decreases and it almost becomes negligible, when the shape 492 parameter is in excess of $m=5$.

493 Fig. 6 depicts the $\mathrm{CP}_{r}$ of the FFR-aided MU-MIMO and 494 SIMO systems at the optimal value of $S_{t h}$ with respect to the tar495 get SINR. The $\mathrm{CP}_{r}$ of FR1 is also plotted for reference. It can be 496 observed in Fig. 6 that the FR1 $\mathrm{CP}_{r}$ is significantly lower 497 than that of FFR-aided MU-MIMO. The $\mathrm{CP}_{r}$ of the FFR-aided 498 SIMO case is higher than that of the FFR-aided MU-MIMO 499 scenario.

500 Fig. 7 plots the average rate of both the FFR and FR1 systems 501 versus the SINR threshold. For plotting the analytical result, 502 (26) and (34) are used for the independent and correlated case, 503 respectively. Observe that the simulation results closely match 504 the analytical results. Firstly, it can be seen that the FFR 505 achieves the maximum value of the average rate at $3.3 \mathrm{~dB}$, which 506 is the $S_{o p t, R}$ value, as shown in Fig. 3 for a $(1 \times 1)$-antenna sys507 tem. Secondly, it can be observed in Fig. 7 that the average rate

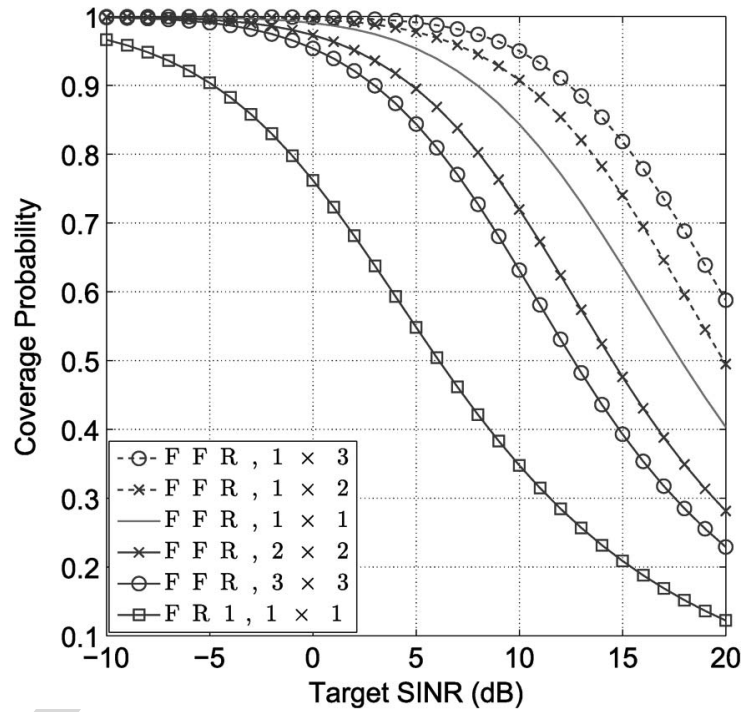

Fig. 6. Coverage probability of both FR1 and of FFR-aided MU-MIMO and SIMO case evaluated for (11) versus the target SINR $T$. Here we have $\alpha=4$ and $S_{t h}=T \mathrm{~dB}, \delta=3$.

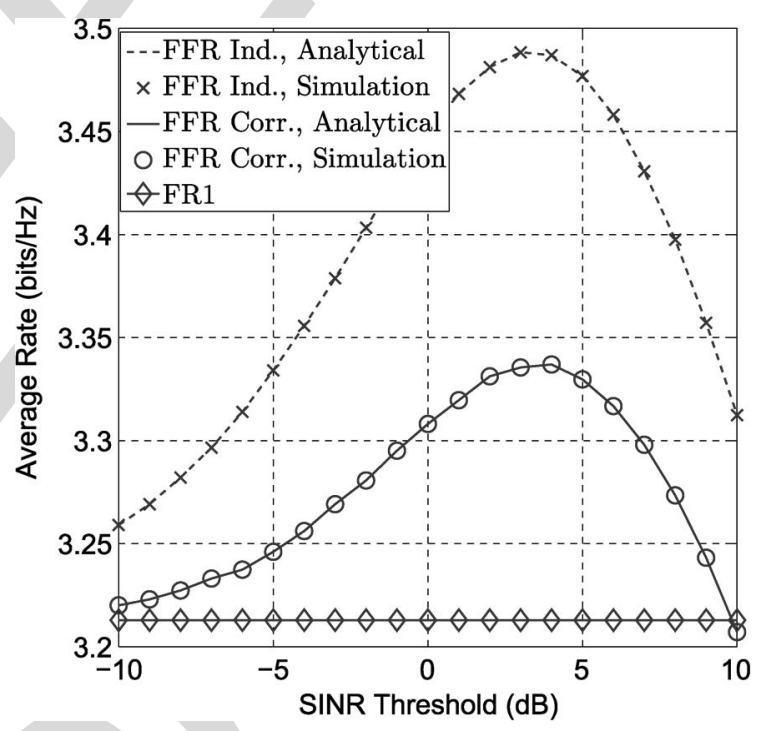

Fig. 7. Average rate of FR 1 and FFR versus the SINR threshold. Here we have $\alpha=4, N_{t}=N_{r}=1$. The theoretical results are plotted from Eq. (26) and (34).

is reduced, when the sub-bands are correlated. Furthermore, 508 interestingly, the optimal SINR threshold of the correlated case 509 is nearly the same as the optimal SINR threshold of the inde- 510 pendent fading case. Although, we have considered continuous 511 log-shaped curve mapping between the SINR and the data rate, 512 in practical scenarios, the mapping is given by discrete curves 513 asscociated with different modulation and coding schemes 514 (MCSs). Therefore, we have also provided the average rate 515 versus the SINR threshold based on the specific MCS level 516 using simulation results as shown in Fig. 8. The mapping 517 between SINR and data rate is based on Table 10.1 of the [34]. It 518 can be observed that the value of $S_{\text {opt }, R}$ is the same as observed 519 in Fig. 7. Furthermore, the optimal SINR threshold of the corre- 520 lated case is nearly the same as the optimal SINR threshold of 521 the independent fading scenario. 


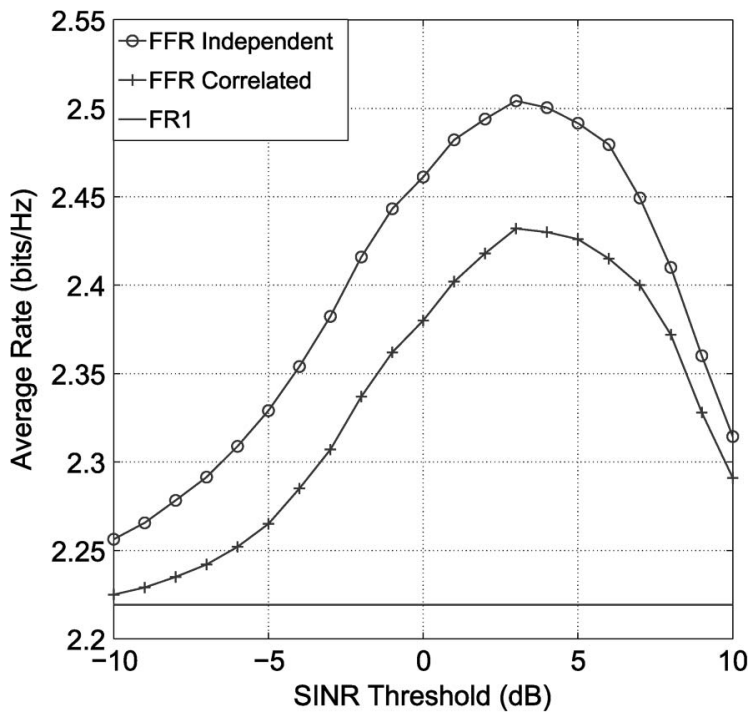

Fig. 8. Average rate of FR1 and FFR using MCS labels versus the SINR threshold. Here we have $\alpha=4, N_{t}=N_{r}=1$.

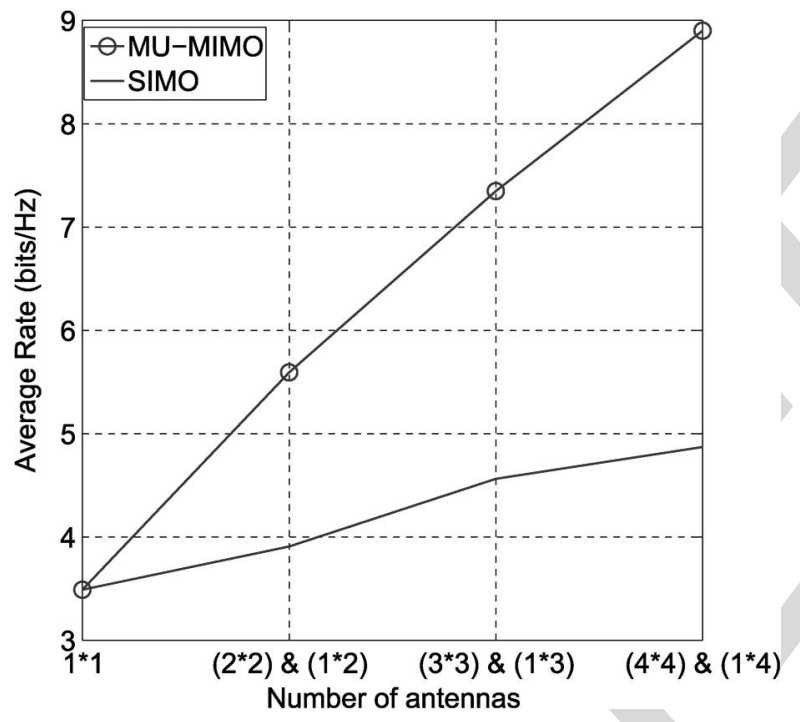

Fig. 9. Maximum average rate achieved by the FFR-aided MU-MIMO and SIMO systems evaluated using (26) and (27) versus the number of antennas for $\alpha=4$.

523 Let us now compare the average rate achieved by the MU524 MIMO and SIMO scenarios at the optimal SINR thresholds. 525 Fig. 9 plots the average rate achieved by the MU-MIMO and 526 SIMO scenarios versus the number of antennas. It is interesting 527 to note that the average rate achieved by the MU-MIMO case 528 is significantly higher than that of the SIMO case. For example, 529 the average rate achieved by the $(2 \times 2)$ MU-MIMO case and 530 by the $(1 \times 3)$ SIMO case are $5.6 \mathrm{bits} / \mathrm{Hz}$ and $4.56 \mathrm{bits} / \mathrm{Hz}$, 531 respectively. In other words, the $(2 \times 2)$ MU-MIMO system 532 achieves a $22.5 \%$ higher rate than the $(1 \times 3)$ SIMO system. 533 However, the overall $\mathrm{CP}_{r}$ achieved by the SIMO case is higher 534 than that of the MU-MIMO case. Now a natural question arises, 535 which of the systems should be chosen by the system designer, 536 since both the $\mathrm{CP}_{r}$ as well as the average rate are important 537 metrics. Based on our results, system designer may opt for the $538(2 \times 2)$ MU-MIMO system over the $(1 \times 3)$ SIMO system, since the gain in average rate is significant and the $\mathrm{CP}_{r}$ degra- 539 dation for $(2 \times 2)$ MU-MIMO is low for lower target SINRs. $\quad 540$

Finally, we have two different expressions for optimal SINR 541 threshold for both the cases, one corresponding to $\mathrm{CP}_{r}\left(S_{t h}=T\right) 542$ and other corresponding to average rate $\left(S_{t h}=T^{\prime}\right)$. To max- 543 imize both $\mathrm{CP}_{r}$ as well as average rate simultaneously, the 544 system designer would have to choose one of these two expres- 545 sions. Now the question arises as to which expression is more 546 appropriate? In order to answer this, we first discuss the benefit 547 of FFR. We see from Figs. 3 and 4 that FFR provides $48 \%$ gain 548 in $\mathrm{CP}_{r}$ and $8.5 \%$ gain in average rate with respect to FR1 at the 549 optimal $S_{t h}$. In other words, FFR provides significantly high 550 gain in $\mathrm{CP}_{r}$ and hence this scheme would be more useful when 551 coverage gain is essentially required. Therefore, FFR-aided 552 MU-MIMO provides both high average rate and satisfactory 553 $\mathrm{CP}_{r}$, since due to MU-MIMO average rate is high and due 554 to FFR scheme $\mathrm{CP}_{r}$ is satisfactory. It can be also noted from 555 Fig. 4 that when $S_{t h}$ is higher than the optimal $S_{t h}$, the loss in 556 $\mathrm{CP}_{r}$ is negligible, while when $S_{t h}$ is lower than the optimal $S_{t h}, 557$ there is significant change in $\mathrm{CP}_{r}$. Hence, for the lower target 558 SINR scenario, i.e., $T<T^{\prime}$, the system designer should choose 559 optimal $S_{t h}$ corresponding to average rate $\left(S_{t h}=T^{\prime}\right)$. On the 560 other hand, for higher target SINR scenario, i.e., $T>T^{\prime}$, the 561 system designer should choose optimal $S_{t h}$ corresponding to $\mathrm{CP}_{r} 562$ $\left(S_{t h}=T\right)$.

\section{CONCLUSION}

564

We have derived expressions for both the $\mathrm{CP}_{r}$ and average 565 rate of MU-MIMO and SIMO systems based on a planned 566 FFR deployment. The impact of frequency-domain correlation 567 between the sub-bands allocated to the FR1 and FR3 regions 568 on the average rate and on the $\mathrm{CP}_{r}$ was analysed in detail, 569 since any practical OFDMA system will typically experience 570 frequency-domain correlation. We analytically determined the 571 optimal SINR threshold, which maximizes the $\mathrm{CP}_{r}$, and also de- 572 termined the optimal SINR threshold (denoted by $S_{\text {opt }, R}$ ), which 573 maximizes the average rate for both the MU-MIMO and SIMO 574 systems considered. It was shown that for the optimal choice 575 of the SINR threshold, the $\mathrm{CP}_{r}$ of the FFR system is higher 576 than that of its FR3 counterpart. The value of $S_{\text {opt }, R}$ increases, 577 when the number of antennas is reduced in a MU-MIMO, where 578 it is assumed that the number of transmit antennas is equal to 579 the number of receive antennas, i.e., $N_{t}=N_{r}=N_{a}$. However, 580 it increases when the number of receive antennas increases in 581 the SIMO scenario. Furthermore, the performance of FFR of 582 the MU-MIMO system and SIMO system are compared. It was 583 shown that $\left(N_{a} \times N_{a}\right)$-element FFR-aided MU-MIMO achieves 584 a significantly higher average rate than $\left(1 \times 2 N_{a}-1\right)$-element 585 SIMO counterpart, but MU-MIMO achieves a lower coverage 586 quality than its SIMO counterpart. However its average rate im- 587 provement is more significant than its $\mathrm{CP}_{r}$ reduction, especially 588 for a lower value of $N_{a}$ and for a lower target SINR. Hence a 589 $(2 \times 2)$ system is preferred over a $(1 \times 3)$ system.

590

A natural extension of this work is to study the FFR-aided 591 MU-MIMO and SIMO system in the context of the cellular 592 uplink [35], [36]. In this study, we have assumed having a 593 fixed transmission power and that the resource blocks are 594 
595 equitably shared by the users. Our future work could consider 596 unequal transmit powers and the unequal allocation of the 597 resource blocks as well as the study of both FFR-aided MU598 MIMO and SIMO systems. Moreover, although strict FFR 599 was considered in the paper, it would also be of substantial 600 interest to study dynamic FFR-aided MU-MIMO and SIMO 601 systems.

602

\section{APPENDIX A}

603 To obtain the $S_{\text {opt }, C}$, we consider the following three possi604 bilities: (i) $S_{t h}<T$, (ii) $S_{t h}=T$, (iii) $S_{t h}>T$.

(i) $S_{t h}<T$ : Let $S_{t h}=T-\Delta$, where $\Delta>0$, then $P_{f}(r)$ can be expressed as in terms of $T$

$$
\begin{aligned}
P_{F}\left(r, S_{t h}<T\right)= & \prod_{i \in \psi}\left(\frac{1}{1+T r^{\alpha} d_{i}^{-\alpha}}\right)^{N_{t}} e^{-T r^{\alpha} \frac{\sigma^{2}}{P}} \\
& +P_{3}(T, r)-P_{3}(T, r) P_{1}(T-\Delta, r) .
\end{aligned}
$$

607 (ii) $S_{t h}=T$ : In this case $P_{f}(r)$ in terms of $T$ can be formu608 lated as

$$
\begin{aligned}
P_{F}\left(r, S_{t h}=T\right)= & \prod_{i \in \psi}\left(\frac{1}{1+T r^{\alpha} d_{i}^{-\alpha}}\right)^{N_{t}} e^{-T r^{\alpha} \frac{\sigma^{2}}{P}} \\
& +P_{3}(T, r)-P_{3}(T, r) P_{1}(T, r) . \\
= & P_{1}(T, r)\left(1-P_{3}(T, r)\right)+P_{3}(T, r) .
\end{aligned}
$$

609 (iii) $S_{t h}>T$ : Let $S_{t h}=T+\Delta$, where $\Delta>0$, then $P_{f}(r)$ in 610 terms of $T$ is given by

$$
\begin{aligned}
P_{F}\left(r, S_{t h}>T\right)= & \prod_{i \in \psi}\left(\frac{1}{1+(T+\Delta) r^{\alpha} d_{i}^{-\alpha}}\right)^{N_{t}} e^{-(T+\Delta) r^{\alpha} \frac{\sigma^{2}}{P}} \\
& +P_{3}(T, r)-P_{3}(T, r) P_{1}(T+\Delta, r) . \\
= & P_{1}(T+\Delta, r)\left(1-P_{3}(T, r)\right)+P_{3}(T, r) .
\end{aligned}
$$

611 Let us now compare the FFR $\mathrm{CP}_{r}$ for $S_{t h}<T$ and $S_{t h}=T$ 612 given by (35) and (36), respectively. Since we have $P_{1}(T-\Delta$, $613 r)>P_{1}(T, r)$, this implies that $P_{F}\left(r, S_{t h}<T\right)<P_{F}\left(r, S_{t h}=T\right)$. 614 Similarly, we compare the FFR-aided $\mathrm{CP}_{r}$ for $S_{t h}=T$ and $615 S_{t h}>T$ given by (37) and (38), respectively. Since $P_{1}(T+\Delta$, $616 r)<P_{1}(T, r)$, this implies that $P_{F}\left(r, S_{t h}=T\right)>P_{F}\left(r, S_{t h}>T\right)$. 617 Thus, FFR achieves the maximum achievable $\mathrm{CP}_{r}$ when $S_{t h}=T$. 618 Note that when one chooses the SINR threshold to be $S_{o p t, C}$, 619 then the $\mathrm{CP}_{r}$ of FFR is higher than that of FR3 since we 620 have $C P_{F}\left(r, S_{t h}=T\right)=P_{1}(T, r)\left(1-P_{3}(T, r)\right)+P_{3}(T, r)>$ $621 P_{3}(T, r)$. The reason for this behaviour is as follows: only users 622 having a low SINR (low fading gain for the desired signal 623 and/or high fading gain for the interfering signal) move to the 624 cell-edge region and they experience a new independent fading 625 gain at the cell-edge region. In other words, the increase in FFR $626 \mathrm{CP}_{r}$ over the $\mathrm{FR} 3 \mathrm{CP}_{r}$ is due to the sub-band diversity gains 627 which is achieved by the system, when the users move from the 628 cell-centre to the cell-edge.

\section{APPENDIX B}

Since a cell-centre user is associated with $\eta_{t}(r)>S_{t h}$, the 630 average rate $R_{c}(r)$ of the cell-centre users of the FFR system can 631 be written as $R_{c}(r)=E\left[\ln \left(1+\eta_{t}(r)\right) \mid \eta_{t}(r)>S_{t h}\right]$ Similarly, 632 since a cell-edge user has $\eta_{t}(r)<S_{t h}$, the average rate $R_{e}(r)$ of 633 the cell-edge users in the FFR system can be written as $R_{e}(r)=634$ $E\left[\ln \left(1+\hat{\eta}_{t}(r)\right) \mid \eta_{t}(r)<S_{t h}\right]$. Now, the average rate $R_{f}(r)$ of the 635 FFR system can be written as

$$
R_{f}(r)=R_{c}(r) P\left[\eta_{t}(r)>S_{t h}\right]+\frac{1}{3} R_{e}(r) P\left[\eta_{t}(r)<S_{t h}\right] .
$$

Here the first term denotes the average rate contributed by the 637 cell-centre users, while the second term denotes the contribu- 638 tion of the cell-edge users. Recall that the frequency reuse $\frac{1}{3}$ is 639 invoked for the cell-edge users. In other words, only one third 640 of the cell-edge frequency $\left(F_{1}+F_{2}+F_{3}\right)$ is used for the cell- 641 edge users and hence the factor $\frac{1}{3}$ is multiplied in the above ex- 642 pression. Using the methods outlined in Section IV-A, 643 $R_{c}(r) P\left[\eta(r)>S_{t h}\right]$ can be written as 644

$$
\begin{aligned}
R_{c}(r) P\left[\eta_{t}(r)>S_{t h}\right] & \left.\left.=\int_{t>0} P\left[\ln \left(1+\eta_{t}(r)\right)>t, \eta_{t}\right) r\right)>S_{t h}\right] \mathrm{dt} \\
& =\int_{t>0} P\left[\eta_{t}(r)>\max \left\{e^{t}-1, S_{t h}\right\}\right] \mathrm{dt} . \quad
\end{aligned}
$$

Using (3) and (4), this can be further simplified to

$R_{c}(r) P\left[\eta_{t}(r)>S_{t h}\right]=\int \prod_{t>0}\left(\frac{1}{1+\max \left\{e^{t}-1, S_{t h}\right\} r^{\alpha} d_{j}^{-\alpha}}\right)^{N_{t}} \mathrm{~d} t$.

Again, similar to Section IV-A, we can write $R_{e}(r)$ as

$$
\begin{aligned}
R_{e}(r) & =\int_{t>0} \frac{P\left[\ln \left(1+\hat{\eta}_{t}(r)\right)>t, \eta_{t}(r)<S_{t h}\right]}{P\left[\eta_{t}(r)<S_{t h}\right]} \mathrm{dt} \\
& =\int_{t>0} \frac{P\left[\hat{\eta}_{t}(r)>\left(e^{t}-1\right), \eta_{t}(r)<S_{t h}\right]}{P\left[\eta_{t}(r)<S_{t h}\right]} \mathrm{dt} .
\end{aligned}
$$

Since $g$ and $\hat{g}$ are i.i.d as well as $h_{i}$ and $\hat{h}_{i}$ are also i.i.d, hence 647 $R_{e}(r)$ can be written as

$$
R_{e}(r)=\int \prod_{t>0}\left(\frac{1}{i \in \phi}\right)^{N_{t}} \mathrm{~d} t
$$

Finally substituting back (41) and (43) into (39) and after aver- 649 aging over the spatial dimension, the average rate of the FFR 650 system is given by

$$
\begin{aligned}
R_{f}=\int_{0}^{R} \int_{t>0}( & \prod_{j \in \psi}\left(\frac{1}{1+\max \left\{e^{t}-1, S_{t h}\right\} r^{\alpha} d_{j}^{-\alpha}}\right)^{N_{t}} \\
& \left.+\frac{1}{3} \prod_{i \in \phi} \frac{P\left[\eta_{t}(r)<S_{t h}\right]}{\left(1+\left(e^{t}-1\right) r^{\alpha} d_{i}^{-\alpha}\right)^{N_{t}}}\right) \mathrm{d} t f_{R}(r) \mathrm{d} r
\end{aligned}
$$




$$
\begin{aligned}
R_{f}=\int_{0}^{R} \int_{t>0}\left(\prod_{j \in \psi}\left(\frac{1}{1+\max \left\{e^{t}-1, S_{t h}\right\} r^{\alpha} d_{j}^{-\alpha}}\right)^{N_{t}}\right. \\
\left.+\frac{1}{3} \prod_{i \in \phi} \frac{P\left[\eta_{t}(r)<S_{t h}\right]}{\left(1+\left(e^{t}-1\right) r^{\alpha} d_{i}^{-\alpha}\right)^{N_{t}}}\right) \mathrm{d} t f_{R}(r) \mathrm{d} r .
\end{aligned}
$$

654 To maximize the rate $R_{f}$, we have to differentiate $R_{f}$ with re655 spect to $S_{t h}$. In order to do that we split the first part of the integ656 rand of $R_{f}$ as given in (46), shown at the bottom of the page. 657 Upon substituting $P\left[\eta_{t}(r)<S_{t h}\right]=1-\prod_{j \in \psi}\left(\frac{1}{1+S_{t h} r^{\alpha} d_{j}^{-\alpha}}\right)^{N_{t}}$ 658 into Eq. (45), $R_{f}$ can be rewritten as given in (47), shown at the bottom of the page. Using Leibniz's rule, ${ }^{6}$ while differentiating 659 $R_{f}$ with respect to $S_{t h}$, we obtain (48), shown at the bottom of 660 the page. Simplifying $\frac{d R_{f}}{d S_{t h}}$ and equating it to zero, one obtains 661 $\frac{d R_{f}}{d S_{t h}}$ as given in (48). The solution of the integral given in (48) 662 gives the optimal $S_{t h}$, namely $S_{o p t, R}$, but obtaining $S_{o p t, R}$ in 663 a closed form is a challenging problem, as the distances $d_{i} \mathrm{~s} 664$ are also a function of $r$. Hence, we find the value of $S_{o p t, R}$ by 665 solving (48) numerically (using Mathematica (or Matlab)). 666 Note that the optimal value of $S_{t h}$ is calculated at the time of 667 network planning with the aid of Mathematica (or Matlab) 668 to obtain the numerical values off line. We have investigated 669 $S_{o p t, R}$ as a function of the path loss exponent, of the number of 670 transmit antennas, etc.

${ }^{6}$ Leibniz's rule states that if $f(x, \theta)$ is a function such that $\frac{d}{d \theta} f(x, \theta)$ exist, and it is continuous, then we have $\frac{d}{d \theta}\left(\int_{a(\theta)}^{b(\theta)} f(x, \theta) d x\right)=\int_{a(\theta)}^{b(\theta)} \frac{d}{d \theta}(f(x, \theta)) d x+$ $f(b(\theta), \theta) \frac{d}{d \theta} b(\theta)-f(a(\theta), \theta) \frac{d}{d \theta} a(\theta)$.

$$
\int_{t>0} \prod_{j \in \psi}\left(\frac{1}{1+\max \left\{e^{t}-1, S_{t h}\right\} r^{\alpha} d_{j}^{-\alpha}}\right)^{N_{t}} \mathrm{~d} t=\int_{t>0}^{\ln \left(1+S_{t h}\right)} \prod_{j \in \psi}\left(\frac{1}{1+S_{t h} r^{\alpha} d_{j}^{-\alpha}}\right)^{N_{t}} \mathrm{~d} t+\int_{\ln \left(1+S_{t h}\right)}^{\infty} \prod_{j \in \psi}\left(\frac{1}{1+\left(e^{t}-1\right) r^{\alpha} d_{j}^{-\alpha}}\right)^{N_{t}} \mathrm{~d} t
$$

$$
\begin{aligned}
& R_{f}=\int_{0}^{R}\left(\prod_{j \in \psi} \frac{\ln \left(1+S_{t h}\right)}{\left(1+S_{t h} r^{\alpha} d_{j}^{-\alpha}\right)^{N_{t}}}+\int_{\ln \left(1+S_{t h}\right)}^{\infty} \prod_{j \in \psi}\left(\frac{1}{1+\left(e^{t}-1\right) r^{\alpha} d_{j}^{-\alpha}}\right)^{N_{t}} \mathrm{~d} t\right. \\
& +\left(1-\prod_{j \in \psi}\left(\frac{1}{1+S_{t h} r^{\alpha} d_{j}^{-\alpha}}\right)^{N_{t}}\right) \underbrace{\frac{1}{3} \int_{t>0} \prod_{i \in \phi}\left(\frac{1}{1+\left(e^{t}-1\right) r^{\alpha} d_{i}^{-\alpha}}\right)^{N_{t}} \mathrm{~d} t}_{K(r)}) f_{R}(r) \mathrm{d} r .
\end{aligned}
$$

$$
\begin{aligned}
& \frac{d R_{f}}{d S_{t h}}=\int_{0}^{R}\left(\frac{\frac{\prod_{j \in \psi}\left(1+S_{t h} r^{\alpha} d_{j}^{-\alpha}\right)^{N_{t}}}{1+S_{t h}}-\ln \left(1+S_{t h}\right) \frac{d}{d S_{t h}}\left(\prod_{j \in \psi}\left(1+S_{t h} r^{\alpha} d_{j}^{-\alpha}\right)^{N_{t}}\right)}{\left(\prod_{j \in \psi}\left(1+S_{t h} r^{\alpha} d_{j}^{-\alpha}\right)\right)^{2 N_{t}}}\right. \\
& -\prod_{j \in \psi} \frac{1}{\left(1+S_{t h} r^{\alpha} d_{j}^{-\alpha}\right)^{N_{t}}}\left(\frac{1}{1+S_{t h}}\right)+\frac{K(r) \frac{d}{d S_{t h}}\left(\prod_{j \in \psi}\left(1+S_{t h} r^{\alpha} d_{j}^{-\alpha}\right)^{N_{t}}\right)}{\left(\prod_{j \in \psi}\left(1+S_{t h} r^{\alpha} d_{j}^{-\alpha}\right)\right)^{2 N_{t}}} f_{R}(r) \mathrm{d} r . \\
& \frac{d R_{f}}{d S_{t h}}=\int_{0}^{R}\left(\frac{\left(K(r)-\ln \left(1+S_{t h}\right)\right) \sum_{i \in \psi}\left(1+S_{t h} r^{\alpha} d_{i}^{-\alpha}\right)^{N_{t}-1} r^{\alpha} d_{i}^{-\alpha}\left(\prod_{j \in \psi \backslash i}\left(1+S_{t h} r^{\alpha} d_{j}^{-\alpha}\right)^{N_{t}}\right)}{\left(\prod_{j \in \psi}\left(1+S_{t h} r^{\alpha} d_{j}^{-\alpha}\right)\right)^{2 N_{t}}}\right) f_{R}(r) \mathrm{d} r=0
\end{aligned}
$$




\section{REFERENCES}

[1] G. Boudreau et al., "Interference coordination and cancellation for $4 \mathrm{G}$ networks," IEEE Commun. Mag., vol. 47, no. 4, pp. 74-81, Apr. 2009.

[2] N. Himayat, S. Talwar, A. Rao, and R. Soni, "Interference management for 4G cellular standards [WIMAX/LTE UPDATE]," IEEE Commun. Mag., vol. 48, no. 8, pp. 86-92, Aug. 2010.

[3] F. Wang et al., "Mobile WiMAX systems: Performance and evolution," IEEE Commun. Mag., vol. 46, no. 10, pp. 41-49, Oct. 2008.

[4] D. Astely et al., "LTE: The evolution of mobile broadband," IEEE Commun. Mag., vol. 47, no. 4, pp. 44-51, Apr. 2009.

5] A. S. Hamza, S. S. Khalifa, H. S. Hamza, and K. Elsayed, "A survey on inter-cell interference coordination techniques in OFDMA-based cellular networks," IEEE Commun. Surveys Tuts., vol. 15, no. 4, pp. 1642-1670, 4th Quart. 2013.

[6] R. Y. Chang, Z. Tao, J. Zhang, and C.-C. J. Kuo, "A graph approach to dynamic Fractional Frequency Reuse (FFR) in multi-cell OFDMA networks," in Proc. IEEE ICC, Jun. 2009, pp. 1-6.

[7] M. Assaad, "Optimal Fractional Frequency Reuse (FFR) in multicellular OFDMA system," in Proc. IEEE 68th VTC-Fall, Sep. 2008, pp. 1-5.

[8] Z. Xu, G. Ye Li, C. Yang, and X. Zhu, "Throughput and optimal threshold for FFR schemes in OFDMA cellular networks," IEEE Trans. Wireless Commun., vol. 11, no. 8, pp. 2776-2785, Aug. 2012.

[9] T. Novlan, J. G. Andrews, I. Sohn, R. K. Ganti, and A. Ghosh, "Comparison of fractional frequency reuse approaches in the OFDMA cellular downlink," in Proc. IEEE Global Telecommun. Conf., 2010, pp. $1-5$.

0] T. D. Novlan, R. K. Ganti, A. Ghosh, and J. G. Andrews, "Analytical evaluation of fractional frequency reuse for OFDMA cellular networks," IEEE Trans. Wireless Commun., vol. 10, no. 12, pp. 4294-4305, Dec. 2011.

1] H. Zhuang and T. Ohtsuki, "A model based on Poisson point process for analyzing MIMO heterogeneous networks utilizing fractional frequency reuse," IEEE Trans. Wireless Commun., vol. 13, no. 12, pp. 6839-6850, Dec. 2014.

2] A. Mahmud and K. A. Hamdi, "A unified framework for the analysis of fractional frequency reuse techniques," IEEE Trans. Commun., vol. 62, no. 10 , pp. 3692-3705, Oct. 2014.

3] D. G. Gonzalez, M. Garcia-Lozano, S. Ruiz Boque, and D. S. Lee, "Optimization of soft frequency reuse for irregular LTE macrocellular networks," IEEE Trans. Wireless Commun., vol. 12, no. 5, pp. 2410-2423, May 2013.

4] X. Tao, F. Xu, W. ur Rehman, Y. Xu, and X. Li, "A generic mathematical model based on fuzzy set theory for frequency reuse in cellular networks," IEEE J. Sel. Areas Commun., vol. 31, no. 5, pp. 861-869, May 2013.

15] L.-C. Wang and C.-J. Yeh, "3-cell network MIMO architectures with sectorization and fractional frequency reuse," IEEE J. Sel. Areas Commun., vol. 29, no. 6, pp. 1185-1199, Jun. 2011.

16] S. Kumar and S. Kalyani, "Impact of correlated interferers on coverage and rate of FFR and SFR schemes," IEEE Trans. Veh. Technol., to be published.

17] H. Chang and I. Rubin, "Optimal downlink and uplink fractional frequency reuse in cellular wireless networks" IEEE Trans. Veh. Technol., to be published.

[18] O. Aliu, M. Mehta, M. Imran, A. Karandikar, and B. Evans, "A new cellular-automata-based fractional frequency reuse scheme," IEEE Trans. Veh. Technol., vol. 64, no. 4, pp. 1535-1547, Apr. 2015.

9] Q. Li, R. Hu, Y. Xu, and Y. Qian, "Optimal fractional frequency reuse and power control in the heterogeneous wireless networks," IEEE Trans. Wireless Commun., vol. 12, no. 6, pp. 2658-2668, Jun. 2013.

20] J. Zhang, R. Zhang, G. Li, and L. Hanzo, "Distributed antenna systems in fractional-frequency-reuse-aided cellular networks," IEEE Trans. Veh. Technol., vol. 62, no. 3, pp. 1340-1349, Mar. 2013.

[21] S. Kumar, S. Kalyani, and K. Giridhar, "Spectrum allocation for ICIC based picocell," IEEE Trans. Veh. Technol., to be published.

[22] F. Jin, R. Zhang, and L. Hanzo, "Fractional frequency reuse aided twin-layer femtocell networks: Analysis, design and optimization," IEEE Trans. Commun., vol. 61, no. 5, pp. 2074-2085, May 2013.

23] W. S. Jeon, J. Kim, and D. G. Jeong, "Downlink radio resource partitioning with fractional frequency reuse in femtocell networks," IEEE Trans. Veh. Technol., vol. 63, no. 1, pp. 308-321, Jan. 2014.

24] F. Wang and W. Wang, "Analytical evaluation of femtocell deployment in cellular networks using fractional frequency reuse," IET Commun., vol. 8, no. 9, pp. 1599-1608, Jun. 2014.

25] N. Saquib, E. Hossain, and D. I. Kim, "Fractional frequency reuse for interference management in lte-advanced hetnets," IEEE Wireless Commun., vol. 20, no. 2, pp. 113-122, Apr. 2013.
[26] J. Y. Lee, S. J. Bae, Y. M. Kwon, and M. Y. Chung, "Interference anal- 748 ysis for femtocell deployment in OFDMA systems based on fractional 749 frequency reuse," IEEE Commun. Lett., vol. 15, no. 4, pp. 425-427, 750 Apr. 2011.

[27] Q. H. Spencer, A. L. Swindlehurst, and M. Haardt, "Zero-forcing methods 752 for downlink spatial multiplexing in multiuser MIMO channels," IEEE 753 Trans. Signal Process., vol. 52, no. 2, pp. 461-471, Feb. 2004.

[28] D. Gesbert, M. Kountouris, R. W. Heath, C.-B. Chae, and T. Salzer, 755 "Shifting the MIMO paradigm," IEEE Signal Process. Mag., vol. 24, 756 no. 5, pp. 36-46, Sep. 2007.

[29] S. Jafar and A. Goldsmith, "Isotropic fading vector broadcast channels: 758 The scalar upper bound and loss in degrees of freedom," IEEE Trans. Inf. 759 Theory, vol. 51, no. 3, pp. 848-857, Mar. 2005.

[30] S. Catreux, P. Driessen, and L. Greenstein, "Simulation results for an 761 interference-limited multiple-input multiple-output cellular system," 762 IEEE Commun. Lett., vol. 4, no. 11, pp. 334-336, Nov. 2000.

[31] J. G. Andrews, W. Choi, and R. W. Heath, "Overcoming interference in 764 spatial multiplexing MIMO cellular networks," IEEE Wireless Commun., 765 vol. 14, no. 6, pp. 95-104, Dec. 2007.

[32] D. Tse and P. Viswanath, Fundamentals of Wireless Communication. 767 Cambridge, U.K.: Cambridge Univ. Press, 2005.

[33] K. B. Baltzis, "Hexagonal vs circular cell shape: A comparative analysis 769 and evaluation of the two popular modeling approximations," in Cellular 770 Networks-Positioning, Performance Analysis, Reliability, A. Melikov Ed. 771 Rijeka, Croatia: Intechopen, 2011.

[34] S. Sesia, I. Toufik, and M. Baker, LTE-The UMTS Long Term Evolution: 773 From Theory to Practice. Hoboken, NJ, USA: Wiley, 2011. [Online]. 774 Available: https://books.google.co.uk/books?id=beIaPXLzYKcC 775

[35] H. Tabassum, F. Yilmaz, Z. Dawy, and M.-S. Alouini, "A framework 776 for uplink intercell interference modeling with channel-based schedul- 777 ing," IEEE Trans. Wireless Commun., vol. 12, no. 1, pp. 206-217, 778 Jan. 2013.

[36] H. Tabassum, Z. Dawy, M. S. Alouini, and F. Yilmaz, "A generic 780 interference model for uplink OFDMA networks with fractional fre- 781 quency reuse," IEEE Trans. Veh. Technol., vol. 63, no. 3, pp. 1491-1497, 782 Mar. 2014.

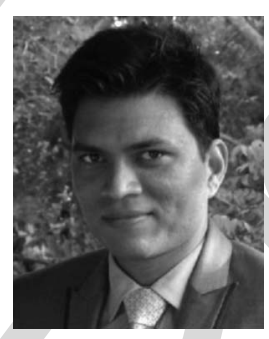

Suman Kumar received the B.Tech. degree in elec- 784 tronics and communication engineering from the 785 Future Institute of Engineering and Management, 786 Kolkata, India, in 2010. He is currently pursuing 787 the Ph.D. degree in the Department of Electrical 788 Engineering, Indian Institute of Technology, Madras, 789 India. He is the recipient of a Best Paper Award at 790 ICWMC-2012 held at Venice, Italy.

His research interests are broadly in the areas of 792 performance analysis of mobile broadband wireless 793 networks including frequency reuse, HetNets, hyper- 794 geometric functions, and generalized fading models.

795

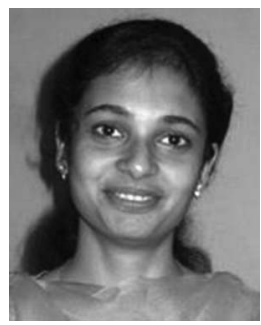

Sheetal Kalyani received the B.E. degree in elec- 796 tronics and communication engineering from the 797 Sardar Patel University, Gujarat, India, in 2002 and 798 the Ph.D. degree in electrical engineering from the 799 Indian Institute of Technology, Madras, India, in 800 2008. She was a Senior Research Engineer in Cen- 801 tre of Excellence in Wireless Technology, Chennai, 802 India, from 2008 to 2012. She is currently an As- 803 sistant Professor in the Department of Electrical 804 Engineering, Indian Institute of Technology, Madras. 805

Her current research interests include HetNets, 806 extreme value theory, hypergeometric functions, generalized fading models, 807 and statistical learning algorithms for prediction. 


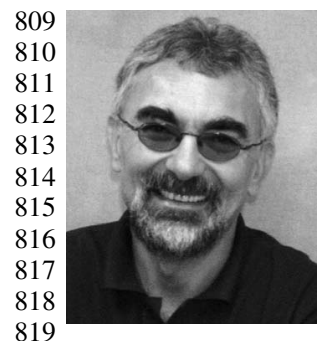

Lajos Hanzo (M'91-SM'92-F'04) received the degree in electronics in 1976 and his doctorate in 1983 from the Technical University of Budapest, Budapest. Hungary. In 2009, he was awarded an honorary doctorate by the Technical University of Budapest, and in 2015 by the University of Edinburgh. During his 38-year career in telecommunications, he has held various research and academic posts in Hungary, Germany, and the U.K. Since 1986, he has been with the School of Electronics and Computer Science, University of Southampton, 820 U.K., where he holds the Chair in telecommunications. He has successfully 821 supervised about 100 Ph.D. students, co-authored 20 John Wiley/IEEE Press 822 books on mobile radio communications totalling in excess of 10000 pages, 823 published over 1500 research entries at IEEE Xplore, acted both as TPC 824 and General Chair of IEEE conferences, presented keynote lectures, and 825 been awarded a number of distinctions. Currently, he is directing a 60826 strong academic research team, working on a range of research projects 827 in the field of wireless multimedia communications sponsored by industry, 828 the Engineering and Physical Sciences Research Council (EPSRC) U.K., 829 the European Research Council's Advanced Fellow Grant, and the Royal 830 Society's Wolfson Research Merit Award. He is an enthusiastic supporter of 831 industrial and academic liaison and he offers a range of industrial courses. $832 \mathrm{He}$ is also a Governor of the IEEE Vehicular Technology Society. During 833 2008-2012, he was the Editor-in-Chief of the IEEE Press and a Chaired 834 Professor also at Tsinghua University, Beijing. His research is funded by 835 the European Research Council's Senior Research Fellow Grant. Dr. Hanzo 836 has over 22000 citations. For further information on research in progress 837 and associated publications, please refer to http://www-mobile.ecs.soton.ac.uk.

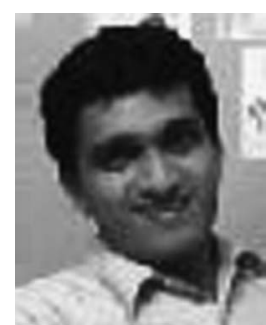

K. Giridhar (M'XX) received the B.Sc. degree in 838 AQ7 applied sciences from PSG College of Technology, 839 Coimbatore, India, the M.E. degree in electrical 840 communications from Indian Institute of Science, 841 Bangalore, India, and the Ph.D. degree in electri- 842 cal engineering from University of California, Santa 843 Barbara, Santa Barbara, CA, USA.

844

$\mathrm{He}$ is a Professor at the Indian Institute of Tech- 845 nology Madras (www.iitm.ac.in), Chennai. During 846 1989 and 1990, he was a Member of Research Staff 847 at CRL, Bharat Electronics, Bangalore, and during 848 1993 and 1994, was a Research Affiliate in electrical engineering at Stanford 849 University, Stanford, CA, USA. Since 1994, he has been with the Department 850 of Electrical Engineering, Indian Institute of Technology, Madras (ITTM). He 851 has been a Visiting Faculty at Sri Sathya Sai Institute of Higher Learning, 852 Prasanthi Nilayam, Andhra Pradesh, and at Stanford University. His research 853 interests are broadly in the areas of adaptive signal processing and wireless 854 communications systems, with an emphasis on various transceiver algorithms, 855 custom air-interface design for strategic applications, and performance analysis 856 of mobile broadband wireless networks including HetNets.

Dr. Giridhar is a member of the Telecommunications and Computer 858 Networks (TeNeT) Group (www.tenet.res.in) at IITM. He actively collaborates 859 with the Center of Excellence in Wireless Technology (www.cewit.org.in) on 860 MIMO-OFDM broadband access research, resulting in several contributions to 861 IEEE $802.16 \mathrm{~m}$, and currently on proposals to LTE-A and 5G forums. He serves 862 as a consultant to many telecom \& VLSI companies in India, and was on a 863 sabbatical in 2004-2005 with Beceem Communications. 


\section{AUTHOR QUERIES}

\section{AUTHOR PLEASE ANSWER ALL QUERIES}

AQ1 = Please provide keywords.

AQ2 = Please provide department name for IIT, Madras.

AQ3 = Please provide publication update in Ref. [16].

AQ4 = Please provide publication update in Ref. [17].

AQ5 = Please provide publication update in Ref. [21].

AQ6 = Please provide page range of chapter for Ref. [33].

AQ7 = Please provide membership history for K. Giridhar.

\section{END OF ALL QUERIES}

